\title{
An Invitation to the Generalized Saturation Conjecture $^{\dagger}$
}

\author{
By \\ Anatol N. KIRILLOV* \\ "Я вам не скажу за всю Одессу, \\ вся Одесса очень велика." \\ From a famous russian song.
}

"I'm not intending to tell all about Tokyo,

The whole Tokyo is too big."

\begin{abstract}
We report about some results, interesting examples, problems and conjectures revolving around the parabolic Kostant partition functions, the parabolic Kostka polynomials and "saturation" properties of several generalizations of the LittlewoodRichardson numbers. The Contents contains the titles of main topics we are going to discuss in the present paper.
\end{abstract}

\section{Contents}

$\S 1 . \quad$ Introduction

§1.1. Notation

Communicated by T. Kawai. Received March 2, 2004. Revised May 17, 2004.

2000 Mathematics Subject Classification(s): 05E05, 05E10, 05A19.

${ }^{\dagger}$ This article is an invited contribution to a special issue of Publications of RIMS commemorating the fortieth anniversary of the founding of the Research Institute for Mathematical Sciences.

* Research Institute for Mathematical Sciences, Kyoto University, Sakyo-ku, Kyoto 606-8502, Japan.

e-mail: kirillov@kurims.kyoto-u.ac.jp; http://www.kurims.kyoto-u.ac.jp/ kirillov

(C) 2004 Research Institute for Mathematical Sciences, Kyoto University. All rights reserved. 
$\S 2 . \quad$ Basic Definitions and Notation

$\S 2.1$. Compositions and partitions

$\S 2.2$. Kostka-Foulkes polynomials

§2.3. Skew Kostka-Foulkes polynomials

$\S 2.4$. Littlewood-Richardson numbers and Saturation Theorem

$\S 2.5$. Internal product of Schur functions, and polynomials $L_{\alpha, \beta}^{\mu}(q)$

$\S 2.6$. Extended and restricted Littlewood-Richardson numbers

$\S 3$. Parabolic Kostant Partition Function and its $q$-analog

§3.1. Definitions: algebraic and combinatorial

§3.2. Elementary properties, and explicit formulas for $l(\eta) \leq 4$

$\S 3.3$. Non-vanishing, Degree and Saturation theorems

$\S 3.4$. Rationality and polynomiality theorems

§3.5. Parabolic Kostant partition function $K_{\Phi(\eta)}(\gamma)$ as function of $\gamma$

§3.6. Reconstruction theorem

$\S 4$. Parabolic Kostka Polynomials: Definition and Basic Properties

$\S 5$. Parabolic Kostka Polynomials: Examples

§5.1. Parabolic Kostka and Kostka-Foulkes polynomials

§5.2. Parabolic Kostka polynomials and Littlewood-Richardson numbers

$\S 5.3$. MacMahon polytope and rectangular Narayana numbers [35]

$\S 5.4$. Gelfand-Tsetlin's polytope $G T\left(\left(2^{k}, 1^{n}\right),\left(1^{2 k+n}\right)\right)$

$\S 5.5$. One dimensional sums and parabolic Kostka polynomials

$\S 6$. Parabolic Kostka Polynomials: Conjectures

§6.1. Non-vanishing conjecture

§6.2. Positivity conjecture

$\S 6.3$. Generalized saturation conjecture for parabolic Kostka polynomials

$\S 6.4$. Rationality conjecture

$\S 6.5$. Polynomiality conjecture

$\S 6.6$. The generalized Fulton, $d(\lambda, \mu \| \eta)=2$ and $d(\lambda, \mu \| \eta)=3$ conjectures

$\S 6.7$. $q$-Log concavity and $P$-positivity conjectures

$\S 6.8$. The generalized Fomin-Fulton-Li-Poon conjectures

$\S 6.9$. Miscellany

References 


\section{$\S 1$ Introduction}

This note is based on a series of lectures given by the author during 19982003 years concerning the interrelations between the saturation properties of the Littlewood-Richardson numbers and their several generalizations, parabolic $q$-Kostant partition functions and parabolic Kostka polynomials.

In spite of the title "An invitation to the Generalized Saturation Conjecture", we will state a big amount of conjectures (about 30) and problems (about 15) revolving around a very mysterious behavior of the coefficients, and the leading term especially, of a parabolic Kostka polynomial.

Remember that, by definition, a function $f: \Omega \subset \mathbb{Z}^{n} \rightarrow \mathbb{Z}$ satisfies the saturation property (on the set $\Omega$ ), if the following condition holds:

$f(N \omega) \neq 0$ for some integer $N \geq 1$ and $\omega \in \Omega$, then also $f(\omega) \neq 0$.

For example, any homogeneous function $f$ on the set $\Omega$, i.e. that satisfying the condition $f(N \omega)=N^{\alpha} f(\omega)$ for some $\alpha \in \mathbb{R}, \forall \omega \in \Omega$ and all integers $N \geq 1$, possesses the saturation property; a subset $\Omega \subset \mathbb{Z}^{n}$ is called saturated if its characteristic function has the saturation property.

To be more specific, let us introduce the numbers $a(\lambda, \mu \| \eta), b(\lambda, \mu \| \eta)$, $c(\lambda, \mu \| \eta)$ and $d(\lambda, \mu \| \eta)$ which will play an important role in our paper. Namely, let $\lambda$ be a partition and $\mu$, and $\eta$ be compositions such that $|\lambda|=|\mu|$ and $l l(\mu) \leq|\eta|$, see Section 2.1 for explanation of notation. Let $K_{\lambda \mu \eta}(q)$ be the corresponding parabolic Kostka polynomial. If $K_{\lambda \mu \eta}(q) \neq 0$, the numbers above are defined from the decomposition

$$
K_{\lambda \mu \eta}(q)=b(\lambda, \mu \| \eta) q^{a(\lambda, \mu \| \eta)}+\cdots+d(\lambda, \mu \| \eta) q^{c(\lambda, \mu \| \eta)},
$$

where we assume that $b(\lambda, \mu \| \eta) \neq 0$ and $d(\lambda, \mu \| \eta) \neq 0$, and $a(\lambda, \mu \| \eta) \leq$ $c(\lambda, \mu \| \eta)$.

If $K_{\lambda \mu \eta}(q)=0$, we put by definition $a(\lambda, \mu \| \eta)=b(\lambda, \mu \| \eta)=c(\lambda, \mu \| \eta)=$ $d(\lambda, \mu \| \eta)=0$.

(*) We expect that $d(\lambda, \mu \| \eta) \geq 0$, and $K_{\lambda \mu \eta}(q) \neq 0$ if and only if $\lambda-\mu \epsilon$ $Y_{\eta}$. In other words, we expect that $K_{\lambda \mu \eta}(q) \neq 0$, if and only if $K_{\Phi(\eta)}(\lambda-\mu)>0$, see Section 6, Positivity and Non-vanishing conjectures.

(\$) We regard the numbers $d(\lambda, \mu \| \eta)$ as a generalization of the LittlewoodRichardson coefficients, see comments after Theorem 1.4, and Section 5.2, $\left(1^{0}\right)$ for explanations.

Problem 1.1. Find combinatorial and/or algebro-geometric interpretations of the numbers $d(\lambda, \mu \| \eta)$. 
Remark 1.2. We expect that for given $\lambda, \mu$ and $\eta$ there exists a rational convex polytope $\Delta_{\lambda, \mu}^{\eta}$ such that the number of integer points inside of $\Delta_{\lambda, \mu}^{\eta}$ is equal to $d(\lambda, \mu \| \eta)$.

One of our main observations is that the saturation property of the leading coefficient $d(\lambda, \mu \| \eta)$, i.e. that

(a) $d(N \lambda, N \mu \| \eta) \neq 0$ for some integer $N \geq 1$ if and only if $d(\lambda, \mu \| \eta) \neq 0$, is an easy consequence (but not conversely !) of the statement that the maximal degree $c(\lambda, \mu \| \eta)$ of $q$ in a parabolic Kostka polynomial $K_{\lambda \mu \eta}(q)$ is a homogeneous degree 1 function of $\lambda$ and $\mu$. In other words, we pose the following conjecture:

\section{Conjecture 1.3 (Generalized Saturation Conjecture).}

Let $\lambda$ be a partition, $\mu$ and $\eta$ be compositions such that $|\lambda|=|\mu|$ and $l l(\mu) \leq|\eta|$. Then the coefficient $c(\lambda, \mu \| \eta)$ is a homogeneous piecewise linear function of $\lambda$ and $\mu$. In particular,

$$
c(N \lambda, N \mu \| \eta)=N c(\lambda, \mu \| \eta)
$$

for any positive integer $N$.

Here $l l(\mu)$ denotes the fake length of a composition $\mu$, see Section 2.1 for the definition.

We would like to note here that, in general, the Generalized Saturation Conjecture ( $G S C$ for short) is false for the numbers $a(\lambda, \mu \| \eta)$, see Examples 4.6.

(W) However, we expect that if $\mu$ is a partition, then the GSC does hold for the numbers $a(\lambda, \mu \| \eta)$.

Conjecture 1.3 is obvious for the Kostka-Foulkes polynomials, since in this case

$$
c\left(\lambda, \mu \|\left(1^{|\lambda|}\right)\right)=n(\mu)-n(\lambda)=\sum_{1 \leq i<l \leq l(\mu)} \min \left(\mu_{i}, \mu_{j}\right)-\sum_{1 \leq i<j \leq l(\lambda)} \min \left(\lambda_{i}, \lambda_{j}\right)
$$

is easily seen to be a homogeneous piecewise linear function of $\lambda$ and $\mu$. However, it seems a difficult problem to prove the $G S C$ in general case, especially to find an explicit piecewise linear formula for the numbers $c(\lambda, \mu \| \eta)$.

Now let us explain briefly a connection between our Generalized Saturation Conjecture and the Saturation Theorem by A. Knutson and T. Tao [43], see also $[4,9,14,65]$ for other proofs. 


\section{Theorem 1.4 (Saturation Theorem [43]).}

Let $\lambda, \mu$ and $\nu$ be partitions such that $|\lambda|+|\mu|=|\nu|$. Then

$c_{N \lambda, N \mu}^{N \nu} \neq 0$ for some integer $N \geq 1$ if and only if $c_{\lambda, \mu}^{\nu} \neq 0$.

Here $c_{\lambda, \mu}^{\nu}$ denotes the Littlewood-Richardson number ( $L R$-number for short) corresponding to the partitions $\lambda, \mu$ and $\nu$, see Section 2.4 for details.

Now we are going to explain how the Saturation Theorem follows from the $G S C$.

First of all, we observe that $c_{\lambda, \mu}^{\nu}=b(\Lambda, R)$ for some partition $\Lambda$ and a dominant sequence of rectangular shape partitions $R$, see Section 2 for the definition of a dominant sequence of partitions. Namely, for given partitions $\lambda=\left(\lambda_{1}, \ldots, \lambda_{r}\right), \mu$ and $\nu$ such that $|\lambda|+|\mu|=|\nu|$, define partition

$$
\Lambda=\left(\mu_{1}+\lambda_{1}, \mu_{1}+\lambda_{2}, \ldots, \mu_{1}+\lambda_{r}, \mu\right),
$$

and a dominant rearrangement $R$ of the sequence of rectangular shape partitions $\widetilde{R}=\left\{\left(\mu_{1}^{\lambda_{1}^{\prime}}\right), \nu\right\}$. Then

(\$) $a(\Lambda, R) \geq \sum_{1 \leq j \leq \mu_{1}} \nu_{j}^{\prime}-|\mu|$, and $a(\Lambda, R)=\sum_{1 \leq j \leq \mu_{1}} \nu_{j}^{\prime}-|\mu|$ if and only if $c_{\lambda, \mu}^{\nu} \geq 1$; in addition, $b(\Lambda, R)=c_{\lambda, \mu}^{\nu}$, see Section 5.2 for details.

In other words, the constant term of the polynomial

$$
K_{\lambda, \mu}^{\nu}(q):=q^{\left(|\mu|-\sum_{1 \leq j \leq \mu_{1}} \nu_{j}^{\prime}\right)} K_{\lambda, R}(q)
$$

is equal to the Littlewood-Richardson number $c_{\lambda, \mu}^{\nu}$. See Sections 5.2 and 6.8 where some results and conjectures about the polynomials $K_{\lambda, \mu}^{\nu}(q)$ and their generalizations $K_{A, B, \theta}^{\nu}(q)$, and $K_{A^{(1)}, \ldots, A^{(k)}, \theta}^{\nu}(q)$, are presented.

The next step is to apply the Duality Theorem for parabolic Kostka polynomials $K_{\lambda, R}(q)$ corresponding to a dominant sequence of rectangular shape partitions $R$, see Section $4,(4.37)$, Duality Theorem. As a corollary, we see that the coefficients $a(\Lambda, R)$ and $c(\Lambda, R)$ satisfy the $G S C$ simultaneously. Hence, it follows from our Theorem 1.5 that

(\$) $a(n \Lambda, n R)=n a(\Lambda, R)$ for any integer $n \geq 1$.

Finally, let us deduce the Saturation Theorem from the above considerations. Indeed, assume that $c_{N \lambda, N \mu}^{N \nu} \neq 0$, then

$$
N a(\Lambda, R)=a(N \Lambda, N R)=N\left(\sum_{1 \leq j \leq \mu_{1}} \nu_{j}^{\prime}-|\mu|\right),
$$

and therefore, $a(\Lambda, R)=\sum_{1 \leq j \leq \mu_{1}} \nu_{j}^{\prime}-|\mu|$. The last equality means that $c_{\lambda, \mu}^{\nu} \neq 0$. 
In fact, our arguments show that in the particular case under consideration, the Generalized Saturation Conjecture is equivalent to the Saturation Theorem. However, our main point is that, conjecturally, the $G S C$ is still valid for any partition $\lambda$ and compositions $\mu$, and $\eta$.

Theorem 1.5 (Saturation Theorem for the numbers $c(\lambda, R)$ ).

Let $\lambda$ be a partition and $R$ be a dominant sequence of rectangular shape partitions. Then

(4) $\quad c(N \lambda, N R)=N c(\lambda, R)$ for any integer $N \geq 1$.

Our proof of Theorem 1.5 is based on an explicit homogeneous piecewise linear formula for the Lascoux-Schützenberger statistics charge, obtained by A. Berenstein and A.N.K., see [36, 38] and a fermionic formula for the parabolic Kostka polynomials $K_{\lambda, R}(q)$ corresponding to a dominant sequence of rectangular shape partitions $R$, see e.g. Section $5.1,\left(5^{0}\right)$. The proof is rather technical and long. We assume to present it in a separate publication.

One of our main results, see Section 4, in support of the GSC in general case is:

Theorem 1.6 (Rationality theorem for parabolic Kostka polynomials, I).

The formal power series

$$
\sum_{n \geq 0} K_{n \lambda, n \mu, \eta}(q) t^{n}
$$

is a rational function in $q$ and $t$ of the form

$$
P_{\lambda \mu \eta}(q, t) / Q_{\lambda \mu \eta}(q, t)
$$

where $P_{\lambda \mu \eta}(q, t)$ and $Q_{\lambda \mu \eta}(q, t)$ are mutually prime polynomials in $q$ and $t$ with integer coefficients, $P_{\lambda \mu \eta}(0,0)=1$.

Moreover,

(\$) the denominator $Q_{\lambda \mu \eta}$ has the following form

$$
Q_{\lambda \mu \eta}(q, t)=\prod_{j \in J}\left(1-q^{j} t\right),
$$

where $J:=J_{\lambda \mu \eta}$ is a finite set of non-negative integer numbers, not necessarily distinct;

(30) $P_{\lambda \mu \eta}(1, t)=(1-t)^{t(\lambda, \mu, \eta)} P_{\lambda \mu \eta}(t)$, where $t(\lambda, \mu, \eta) \in \mathbb{Z}_{\geq 0}$, and $P_{\lambda \mu \eta}(t)$ is a polynomial with non-negative integer coefficients. 
Problem 1.7. Find combinatorial and algebro-geometric interpretations of the set $J_{\lambda \mu \eta}$ and the polynomial $J_{\lambda \mu \eta}(q)=\sum_{j \in J_{\lambda \mu \eta}} q^{j}$. bers).

Corollary 1.8 (Polynomiality theorem for parabolic Kostka num-

Let $\lambda$ be a partition and $\mu, \eta$ be compositions such that $|\lambda|=|\mu|$ and $l l(\mu) \leq$ $|\eta|$. Then there exists a polynomial $\mathcal{K}_{\lambda \mu \eta}(t)$ with rational coefficients such that for all integers $N \geq 1$

$$
\mathcal{K}_{\lambda \mu \eta}(N)=K_{N \lambda, N \mu, \eta}(1)
$$

\section{numbers).}

Corollary 1.9 (Polynomiality theorems for Kostka and LR-

(i) Let $\lambda$ be a partition and $\mu$ be a composition of the same size, then the Kostka number $K_{N \lambda, N \mu}(1)$ is a polynomial in $N$ with rational coefficients.

(ii) Let $\lambda, \mu$ and $\nu$ be partitions, then the Littlewood-Richardson number $c_{N \lambda, N \mu}^{N \nu}$ is a polynomial in $N$ with rational coefficients.

See Section 4, Theorem 4.14 and Corollary 4.15. We also give a multivariable generalization of Theorem 1.6, see Theorem 4.17.

We want to emphasize here that the polynomiality property of the functions $N \rightarrow K_{N \lambda, N \mu, \eta}(1)$ and $N \rightarrow c_{N \lambda, \mathbb{N} \mu}^{N \nu}$ is an easy consequence of our Theorem 1.6, but not conversely: one has to check that the (irredundant) denominator $Q_{\lambda \mu \eta}(q, t)$ doesn't have factors of the form $\left(1-q^{k} t^{l}\right)$ with $l \in$ $\mathbb{Z}_{>1}$.

Conjecture 1.10. If $\mu$ is a partition, then the polynomial $\mathcal{K}_{\lambda \mu \eta}(t)$ has non-negative rational coefficients.

See Section 6, Conjecture 6.10, $(\downarrow)$, for more general conjectures concerning the numbers $d(\lambda, \mu \| \eta)$.

We would like to remark that the $G S C$ does not follow immediately from Theorem 1.6, see Section 6, Rationality Conjecture, for details.

The polynomials $P_{\lambda \mu \eta}(q, t)$ may have negative coefficients, and rather difficult to compute. For example, we don't know the explicit formula for polynomial $P_{\left(2^{6}\right),\left(1^{12}\right),\left(1^{12}\right)}(q, t)$. We expect that the polynomials $P_{\lambda \mu \eta}(q, t)$ should have nice algebraic and algebro-geometric interpretations.

Our proof of Theorem 1.6 is a pure algebraic and is based on the study of the parabolic $q$-Kostant partition functions, see Section 3.

Corollary 1.9,(i), has been proved independently by W. Baldoni-Silva and M.Vergne [2], S. Billey, V. Guillemin and E. Rassart [8], ... . Corollary 1.9,(ii), 
has been proved independently by A Knutson (unpublished), H. Derksen and J. Weyman [15], E. Rassart [61], ... .

The main subject of investigation of our paper is the study of interrelations between the saturation properties of the $L R$-numbers and their generalizations, and the leading coefficient of the parabolic Kostka polynomials.

The paper does not contain complete proofs of the main theorems. Our goal is different. The primary purpose of this note is to collect together several results, conjectures and examples revolving around a mysterious behavior of the initial and the leading terms of a parabolic Kostka polynomial.

Let us say a few words about the content of our paper.

In Section 2 we collect together a few definitions and notation which will be frequently used in the subsequent Sections.

In Section 2.1 we remember the definitions of partitions and compositions and some operations over them. We would like to point out here some nonstandard conventions about partitions and compositions used in our paper. We will denote by $\lambda=\left(\lambda_{1}, \ldots, \lambda_{r}\right)$ a (proper) partition, so that if $\lambda \neq \emptyset$, then $\lambda_{r} \neq 0$. We always use $\eta$ to denote a composition without zero components. Contrary, we will use $\mu$ to denote a composition or partition with zero components and zeroes at the end allowed. A typical example is $\mu=(0,2,0,1,3,0,0)$. Thus, according to our conventions, the compositions $(0),(0,0), \ldots$ are different and different from the empty composition $\emptyset$.

In Sections 2.2 and 2.3 we recall the definitions of Kostka-Foulkes and skew Kostka-Foulkes polynomials. For more details, see [10, 16, 29, 35, 41, 42].

In Section 2.4 we remember the definition of the Littlewood-Richardson numbers and state the Saturation Theorem, which has been proved by A. Knutson and T. Tao [43].

We refer the reader to interesting and clearly written papers by W. Fulton $[20,21]$ for detailed account to the so-called Horn problem and its connections with the Saturation Theorem.

In Section 2.5 we study the saturation properties of the internal product structural constants $g_{\alpha \beta \gamma}$ and those of the plethysm $a_{\mu, W}^{\pi}$. It is well-known that the $L R$-numbers $c_{\lambda, \mu}^{\nu}$ are a special case of the internal product structural constants $g_{\alpha \beta \gamma}$, and in turn, the numbers $g_{\alpha \beta \gamma}$ are a special case of the plethysm structural constants $a_{\mu, W}^{\pi}$, see Remark 2.13. However, based on examples we 
arrived at the conclusion that, in the general case, both the numbers $g_{\alpha \beta \gamma}$ and $a_{\mu, W}^{\pi}$ do not satisfy the saturation property.

(*) Nevertheless, we expect that

- the numbers $a_{\mu, W}^{\pi}$ satisfy a weak form of Saturation Conjecture, i.e. for any finite dimensional $\mathfrak{g} l_{n}$-module $W$ there exists a polynomial $p_{W}(t)\left(p_{W}(t)=\right.$ $t$ ??) such that for all partitions $\pi$ and $\mu$ one has

if $a_{N \mu, W}^{N \pi} \geq p_{W}(N)$, then $a_{\mu, W}^{\pi} \neq 0$.

- for an interesting family of polynomials $L_{\alpha, \beta}^{\mu}(q)$ a certain analog of the $G S C$ does hold, see Conjecture 2.22.

It seems an interesting problem to study whether or not the $G S C$ is valid for polynomials $M_{\mu, W}^{\pi}(q)$ which are defined via the decomposition of the plethysm $W \circ s_{\mu}$ :

$$
\left(W \circ s_{\mu}\right)(X)=\sum_{\pi} M_{\mu, W}^{\pi}(q) P_{\pi}(X, q),
$$

where $X=\left(x_{1}, \ldots, x_{n}\right)$, and $P_{\pi}(X, q)$ stands for the Hall-Littlewood polynomials.

In Section 2.5 we also state several results about polynomials $L_{\alpha, \beta}^{\mu}(q)$ and give a few examples supporting our conjectures.

In Section 2.6 we define the extended Littlewood-Richardson numbers as well as the level $l$ extended $L R$-numbers. The latter are a natural generalization of the restricted $L R$-numbers.

( We expect that Saturation Theorem, the strong $q$-log concavity and Fomin-Fulton-Li-Poon's conjectures I and II are still valid for the level $l$ extended $L R$-numbers.

In Section 3 we study some algebraic properties of the parabolic $q$-Kostant partition function $K_{\Phi(\eta)}(\gamma \mid q)$, mainly in a connection with the saturation properties of the latter. For polynomials $K_{\Phi(\eta)}(\gamma \mid q)$ we prove an analog of the $G S C$, Rationality and Polynomiality theorems, and a new recurrence relation. Our proof of Rationality theorem is based on the following simple observation:

Lemma 1.11. Let $R(X, q) \in \mathbb{Q}[q] \llbracket X^{ \pm 1} \rrbracket$ be a rational function in $q$ and $X=\left(x_{1}^{ \pm 1}, \ldots, x_{n}^{ \pm 1}\right)$. Let

$$
R(X, q)=\sum_{m \in \mathbb{Z}^{n}} A_{m}(q) X^{m}
$$

be a Laurent series expansion of $R(X, q)$. 
Let $a_{1}, \ldots, a_{k} \in \mathbb{Z}^{n}$ be fixed, then

$$
\sum_{\left(N_{1}, \ldots, N_{k}\right) \in \mathbb{Z}_{\geq 0}^{k}} A_{N_{1} a_{1}+\cdots+N_{k} a_{k}}(q) x_{1}^{N_{1}} \ldots x_{k}^{N_{k}}
$$

is a rational function in $q$ and $x_{1}, \ldots, x_{k}$.

In Section 3 we also study the parabolic Kostant partition function $K_{\Phi(\eta)}$ $(\gamma)$ as a function of $\gamma$, see Theorems 3.23 and 3.25.

A detailed treatment of the properties of the parabolic $q$-Kostant and Kostant partition functions lies at the heart of the approach to the GSC and to the study of parabolic Kostka polynomials, presented in this paper. However, making an effort to keep the paper in a reasonable size, we do not intend to consider in Section 3, and decided to postpone for subsequent publications, many very interesting aspects of the theory of parabolic Kostant partition function $K_{\Phi(\eta)}(\gamma):=\left.K_{\Phi(\eta)}(\gamma \mid q)\right|_{q=1}$ such as

(i) The special values of parabolic Kostant partition function, see $[2,34,35,68]$;

(ii) Connections with the flow polytopes, see [2, 68];

(iii) Connections with the Orlik-Solomon and Gelfand-Varchenko algebras, [37];

(iv) A $q$-analog of the generalized Kostant partition function, see [68].

In Section 4 we study, mainly, the "saturation properties" of parabolic Kostka polynomials. Many examples, results and conjectures concerning with the parabolic Kostka polynomials, have been already considered in our paper [35]. For the reader's convenience, in the present paper we remember some basic properties of the parabolic Kostka polynomials $K_{\lambda \mu \eta}(q)$, and give a sketch of proofs of Rationality and Polynomiality theorems for the latter, see Theorems 4.14 and 4.17, and Corollaries 4.15, 4.18 and 4.19.

In the case when $\mu$ and $\eta$ correspond to a dominant sequence of rectangular shape partitions $R$, we have obtained the following result:

Theorem 1.12 (Polynomiality theorem for the numbers $b(\lambda, R)$ ).

Let $\lambda$ be a partition and $R$ be a dominant sequence of rectangular shape partitions, then

(\$) $b(N \lambda, N R)$ is a polynomial in $N$ with rational coefficients. 
Our proof of Theorem 1.6 is a largely algebraic, whereas that of Theorem 1.12 is based on a fermionic formula for the parabolic Kostka polynomials $K_{\lambda, R}(q)$.

( We expect that if $\mu$ is a partition, then $b(N \lambda, N \mu \| \eta)$ is a polynomial in $N$ with non-negative rational coefficients, see Section 6 , Polynomiality conjecture, for a more detailed statement.

However, in general, $b(N \lambda, N \mu \| \eta)$ becomes a polynomial in $N$ only starting from big enough $N$, see Section 6, Conjecture 6.10, ( $\downarrow \downarrow)$, and Remark 6.16.

In Section 4 we also study some natural multivariable analogues of Theorem 1.6, and Corollaries 1.7 and 1.8. In particular, we give a sketch of proof of a theorem that for any sequences of partitions $\lambda^{(1)}, \ldots, \lambda^{(k)}$ and compositions $\mu^{(1)}, \ldots, \mu^{(k)}$ the formal power series

$$
\sum_{\left(N_{1}, \ldots, N_{k}\right) \in \mathbb{Z}_{\geq 0}^{k}} K_{N_{1} \lambda^{(1)}+\cdots+N_{k} \lambda^{(k)}, N_{1} \mu^{(1)}+\cdots+N_{k} \mu^{(k)}, \eta}(q) x_{1}^{N_{1}} \ldots x_{k}^{N_{k}}
$$

is a rational function in $q$ and $x_{1}, \ldots, x_{k}$, which has the denominator of some special form, see Section 4, Theorem 4.17.

However, in general, if $k \geq 2$, the functions

$$
\begin{gathered}
\left(N_{1}, \ldots, N_{k}\right) \rightarrow K_{N_{1} \lambda^{(1)}+\cdots+N_{k} \lambda^{(k)}, N_{1} \mu^{(1)}+\cdots+N_{k} \mu^{(k)}, \eta}(1), \quad \text { and } \\
\left(N_{1}, \ldots, N_{k}\right) \rightarrow c_{N_{1} \lambda^{(1)}+\cdots+N_{k} \lambda^{(k)}, N_{1} \mu^{(1)}+\cdots+N_{k} \mu^{(k)}}^{N_{1} \nu^{(1)}+\cdots+N_{k} \nu^{(k)}}
\end{gathered}
$$

are only piecewise polynomial functions on the set $\left\{\left(N_{1}, \ldots, N_{k}\right) \in \mathbb{Z}_{\geq 0}^{k}\right\}$, see Example 4.23.

We want to emphasize here that the special form of the denominator of the rational function $\sum_{\left(N_{1}, \ldots, N_{k}\right) \in \mathbb{Z}_{\geq 0}^{k}} K_{N_{1} \lambda^{(1)}+\cdots+N_{k} \lambda^{(k)}, N_{1} \mu^{(1)}+\cdots+N_{k} \mu^{(k)}, \eta}(1)$, see Theorem 4.17 , ( $)$, is (in our opinion) a key fact to explain a piecewise polynomiality of the "mixed" Kostka numbers $K_{N_{1} \lambda^{(1)}+\cdots+N_{k} \lambda^{(k)}, N_{1} \mu^{(1)}+\cdots+N_{k} \mu^{(k)}, \eta}(1)$ and "mixed" Littlewood-Richardson coefficients.

( N) Nevertheless, we expect that in the case of parabolic Kostant's partition functions, the function $\left(n_{1}, \ldots, n_{k}\right) \rightarrow K_{\Phi(\eta)}\left(n_{1} \gamma_{1}+\cdots+n_{k} \gamma_{k}\right)$ is a polynomial one on the whole set $\left\{\left(n_{1}, \ldots, n_{k}\right) \in \mathbb{Z}_{\geq 0}^{k}\right\}$.

It is well-known that the Kostka-Foulkes number $K_{\lambda \mu}(1)$ counts the number of integral points in some rational convex polytope, the so-called GelfandTsetlin polytope $G T(\lambda, \mu)$. In this connection we would like to pose the following question (cf with mixed lattice point enumerator theorem for integer convex polytopes by P.McMullen [54], or Example 4.23) :

Question 1.13. Let $\Delta_{1}, \ldots, \Delta_{k} \in \mathbb{Q}^{d}$ be rational convex polytopes, and $L: \mathbb{Z}^{d} \rightarrow \mathbb{Z}_{\geq 0}$ be a continuous piecewise linear function. 
Under what assumptions on $L$ and polytopes $\Delta_{1}, \ldots, \Delta_{k}$ the denominator of rational function

$$
\sum_{\left(N_{1}, \ldots, N_{k}\right) \in \mathbb{Z}_{\geq 0}^{k}}\left\{\sum_{a \in\left(N_{1} \Delta_{1}+\cdots+N_{k} \Delta_{k}\right) \cap \mathbb{Z}^{d}} q^{L(a)}\right\} x_{1}^{N_{1}} \ldots x_{k}^{N_{k}}
$$

has only the factors of the form $\left(1-q^{a_{J}^{(j)}} x_{J}\right)$, where $J \subset[1, \ldots, k], x_{J}:=$ $\prod_{j \in J} x_{j}$, and $a_{J}^{(j)}$ are some non-negative integers?

In Section 4, Remark 4.24, we state some preliminary results about the behavior of the parabolic Kostka number $K_{\lambda \mu \eta}(1)$ considered as a function of $\lambda$ and $\mu$ on "the space of parameters" $Z_{\eta}=\left\{(\lambda, \mu) \in \mathbb{Z}_{\geq 0}^{n} \times \mathbb{Z}_{\geq 0}^{n} \mid \lambda_{1} \geq\right.$ $\left.\cdots \geq \lambda_{n}, \lambda-\mu \in Y_{\eta}\right\}$. Based on the properties of the parabolic Kostant partition function, see Section 3, Theorem 3.25, one can show that on the set $Z_{\eta}$ the parabolic Kostka number $K_{\lambda \mu \eta}(1)$ is a continuous piecewise polynomial function in $\lambda_{1}, \ldots, \lambda_{n}$ and $\mu_{1}, \ldots, \mu_{n}$. The main problem about the function $(\lambda, \mu) \rightarrow K_{\lambda \mu \eta}(1)$ we are interested in, is to describe "the dominant chamber" for the latter function, i.e. to describe the maximal domain $Z_{\eta}^{++}$in the set $Z_{\eta}^{+}:=\left\{(\lambda, \mu) \in Z_{\eta} \mid \lambda-\mu \in Y_{\eta}^{+}\right\}$such that $\left.K_{\lambda \mu \eta}(1)\right|_{Z_{\eta}^{++}}=K_{\Phi(\eta)}(\lambda-\mu)$.

In Section 4 we also introduce the parabolic Hall-Littlewood polynomials $Q_{\mu, \eta}(X ; q)$, and state the rationality theorem for the latter, see Remark 4.35. Details and proofs will appear in a separate publication. Finally, we note that for the Kostka-Macdonald polynomials $K_{\lambda, \mu}(q, t)$, see [53], Chapter VI, Section 8 , for the definition, the generating function

$$
Z_{\lambda, \mu}(q, t, x):=\sum_{n \geq 0} K_{n \lambda, n \mu}(q, t) x^{n}
$$

is a formal power series, which is not, in general, a rational function in $q, t$ and $x$.

It seems a very interesting problem to study the properties of the function $Z_{\lambda, \mu}(q, t, x)$, especially in connections with the characters of affine Lie algebras of type $A$ and the Virasoro algebra.

In Section 5 we collect together several examples which might help to illuminate a mysterious nature of the leading term of a parabolic Kostka polynomial. See the Contents of Section 5 for exposing with the list of these examples. In particular, we show that the one dimensional sums (1D-sums for short) which frequently appear in Statistical Mechanics, see e.g. [22, 46] and the literature quoted therein, are a special case of the parabolic Kostka polynomials $K_{\lambda \mu \eta}(q)$ 
corresponding to a rectangular shape partition $\lambda$, see Section 5.5 for details. In Section 5.1 we give, among other things, a few comments about the Merris conjecture, and in Section 5.4 that about the $L R$-numbers $c_{\delta_{n}, \delta_{n}}^{\lambda}$.

In Section 6 we state a few conjectures about the coefficients $a(\lambda, \mu \| \eta)$, $b(\lambda, \mu \| \eta), c(\lambda, \mu \| \eta)$ and $d(\lambda, \mu \| \eta)$. In particular, we expect, see Conjectures $6.14,6.17$ and 6.23 , that

- (The generalized Fulton conjecture)

If $d(n \lambda, n \mu \| \eta)=1$ for some integer $n \geq 1$, then $d(N \lambda, N \mu \| \eta)=1$ for all $N \in \mathbb{Z}_{\geq 1}$.

- (Generalized $d(\lambda, \mu \| \eta)=2$ conjecture)

If $d(n \lambda, n \mu \| \eta)=n+1$ for some integer $n \geq 1$, then $d(N \lambda, N \mu \| \eta)=N+1$ for all $N \in \mathbb{Z}_{\geq 1}$.

- (Generalized $d(\lambda, \mu \| \eta)=3$ conjecture)

(i) If $d(n \lambda, n \mu \| \eta)=2 n+1$ for some integer $n \geq 2$, then $d(N \lambda, N \mu \| \eta)=$ $2 N+1$ for all $N \in \mathbb{Z}_{\geq 1}$;

(ii) If $d(n \lambda, n \mu \| \eta)=\left(\begin{array}{c}n+2 \\ 2\end{array}\right)$ for some integer $n \geq 2$, then $d(N \lambda$, $N \mu \| \eta)=\left(\begin{array}{c}N+2 \\ 2\end{array}\right)$ for all $N \in \mathbb{Z}_{\geq 1}$.

These two cases exhaust the all possibilities when $d(\lambda, \mu \| \eta)=3$.

- (q-Log concavity conjecture)

Let $\lambda$ be a partition and $R$ be a dominant sequence of rectangular shape partitions, then for any integer $n \geq 1$,

$$
\left(K_{n \lambda, n R}(q)\right)^{2} \geq K_{(n-1) \lambda,(n-1) R}(q) K_{(n+1) \lambda,(n+1) R}(q) .
$$

See Section 6.7, Conjecture 6.17, for a more general and detailed statement of the latter conjecture.

- (The generalized Fomin-Fulton-Li-Poon's conjecture I, cf [60], Conjecture 1, [18], Conjecture 2.7)

$$
K_{\widetilde{A}^{(1)}, \ldots, \widetilde{A}^{(k)}, \theta}^{\nu}(q) \geq K_{A^{(1)}, \ldots, A^{(k)}, \theta}^{\nu}(q) .
$$

- (The generalized Fomin-Fulton-Li-Poon's conjecture II, cf [18], Conjecture 5.1) $)^{1}$

$$
K_{A^{*}, B^{*}, \theta}^{\nu}(q) \geq K_{A, B, \theta}^{\nu}(q) .
$$

\footnotetext{
${ }^{1}$ As we learned from the referee, the extension of the original Fomin-Fulton-Li-Poon conjecture II, [18],Conjecture 5.1, to the case of skew diagrams was also stated by F. Bergeron, R. Biagnoli and M. Rosas, see e.g. [6, 7]; see also [55]. The paper [7] contains, among other things, many interesting results in support of the FFLP-conjecture.
} 
See Section 6.8, Conjecture 6.24, for the explanation of notation we have used, further details and more conjectures.

In the case of the $L R$-numbers the Fulton conjecture has been proved in [44].

Some special cases of the Fomin-Fulton-Li-Poon conjecture II have been proved in [18].

Problem 1.14. When does the number $d(\lambda, \mu \| \eta)$ equal to 1 ?

Finally, we would like to remark that our approach to the $G S C$ is purely algebraic and combinatorial. It seems a very interesting problem to find an algebro-geometric explanation of a still experimental observation that the coefficient $c(\lambda, \mu \| \eta)$ is a homogeneous piecewise linear function of $\lambda$ and $\mu$. In this connection we would like to pose the following questions:

Question 1.15 (Parabolic Kostka polynomials and semi-invariants of quivers).

Let $\lambda$ be a partition and $\mu$, and $\eta$ be compositions such that $|\lambda|=|\mu|$ and $l l(\mu) \leq|\eta|$.

Does there exist a quiver $Q$, dimensional vector $\beta$ and $G L(Q, \beta)$-weight $\sigma$ such that

$$
\operatorname{dim} S I(Q, \beta)_{n \sigma}=d(n \lambda, n \mu \| \eta)
$$

for all integers $n \geq 1$ ?

Here $S I(Q, \beta)_{\sigma}$ stands for the weight $\sigma$ subspace of the ring of semiinvariants

$$
S I(Q, \beta):=\mathbb{Q}[\operatorname{Rep}(Q, \beta)]^{S L(Q, \beta)} .
$$

See [14] and [15], and the literature quoted therein, for more details about the ring of semi-invariants of a quiver. It seems a very interesting problem to find an interpretation of the numbers $c(\lambda, \mu \| \eta)$ and $d(\lambda, \mu \| \eta)$ in terms of quivers.

Question 1.16 (A q-analog of $\operatorname{dim} S I(Q, \beta)$ ).

Does there exist a natural filtration

$$
\left\{0=\mathcal{F}_{0} \subset \mathcal{F}_{1} \subset \ldots\right\}
$$

on the ring of semi-invariants $S I(Q, \beta)$ such that for a special quiver $Q=$ $T_{n, n, n}$ and a special dimensional vector $\beta$, see [14], Section 3 ,

$$
\sum_{j \geq 1} \operatorname{dim}\left(\mathcal{F}_{j} / \mathcal{F}_{j-1}\right) q^{j} \doteq c_{\lambda, \mu}^{\nu}(q) ?
$$


Here $c_{\lambda, \mu}^{\nu}(q)$ denotes the $q$-analog of the LR-numbers, see e.g. [11, 49]; for the meaning of the symbol "ㄹ", see Section 1.1.

We would like to end this Introduction by the following remark. Throughout the paper we use the term Conjecture to mean a statement for which we do not have a proof, but which we have checked on a big body of examples (except for Conjectures from Section 6.9). On the other hand, we use an expression "We expect that ..." to mean a statement which we believe is bound to be true, but for which we don't have the extensive supporting evidence. Of course, not all plausible conjectures and reasonable guesses prove to be true. For example, see Remark 4.22.

\section{$\S 1.1$. Notation}

Throughout the paper we follow Macdonald's book [53] as for notation related to the theory of symmetric functions, and Stanley's book [67] as for notation related to Combinatorics. Below we give a list of some special notation which we will frequently use.

1) If $P(q)$ and $Q(q)$ are polynomials in $q$, the symbol $P(q) \doteq Q(q)$ means that the ratio $P(q) / Q(q)$ is a power of $q$.

2) If $a, k_{0}, \ldots, k_{m}$ are (non-negative) integers, the symbol $q^{a}\left(k_{0}, \ldots, k_{m}\right)$ stands for the polynomial $\sum_{j=0}^{m} k_{j} q^{a+j}$.

3) We use the capital Latin letters $A, B, C, \ldots$ to denote the skew diagrams/shapes, and the small or capital Greek letters $\alpha, \beta, \gamma, \lambda, \mu, \Lambda, M, \ldots$ to denote either partitions or compositions.

4) Let $\eta_{1}=\left(\eta_{1,1}, \eta_{1,2}, \ldots, \eta_{1, p}\right)$ and $\eta_{2}$ be compositions, we say that $\eta_{2}$ is a subdivision of $\eta_{1}$, if there exists a sequence of partitions $\mu^{(j)}, 1 \leq j \leq p$, such that $\left|\mu^{(j)}\right|=\eta_{1, j}$ and $\eta_{2}=\left(\mu^{(1)}, \ldots, \mu^{(p)}\right)$.

5) Let $P_{1}(q)$ and $P_{2}(q)$ be polynomials with real coefficients. By definition, the inequality $P_{1}(q) \geq P_{2}(q)$ means that the difference $P_{1}(q)-P_{2}(q)$ is a polynomial with non-negative real coefficients.

\section{§2. Basic Definitions and Notation}

\section{$\S 2.1$. Compositions and partitions}

A composition

$$
\mu=\left(\mu_{1}, \mu_{2}, \ldots, \mu_{r}\right)
$$


is a sequence of non-negative integers. The number $r$ in (2.1) is called the fake length of the composition $\mu$, and denoted by $l l(\mu)$. In the sequel, it will be convenient for us to distinguish between two such sequences which differ only by a string of zeros at the end. Thus, for example, we regard $(2,0,1),(2,0,1,0),(2,0,1,0,0), \ldots$, as different compositions. The size of a composition $\mu$ is defined to be $|\mu|=\mu_{1}+\cdots+\mu_{r}$.

By definition, a composition $\lambda=\left(\lambda_{1}, \lambda_{2}, \ldots, \lambda_{p}\right)$ is called partition, if additionally it satisfies the following condition:

$$
\lambda_{1} \geq \lambda_{2} \geq \cdots \geq \lambda_{p} \geq 0 .
$$

The non-zero $\lambda_{i}$ in (2.2) are called the parts of $\lambda$. The number of parts is the length of $\lambda$, denoted by $l(\lambda)$. Thus, we have $l(\lambda) \leq l l(\lambda):=p$. As in the case of compositions, we distinguish between two sequences (2.2) if they differ only by a string of zeros at the end. If $|\lambda|=n$ we say that $\lambda$ is a partition of $n$. Denote by $\mathcal{P}_{n}$ the set of all partitions of $n$.

A partition $\lambda=\left(\lambda_{1}, \lambda_{2} \ldots, \lambda_{p}\right)$ is called proper if $\lambda_{p} \neq 0$.

The dominance partial ordering " $\geq$ " on the set of compositions of the same size $n$, or that of partitions $\mathcal{P}_{n}$, is defined as follows:

$$
\begin{aligned}
& \lambda \geq \mu \text { if and only if } \\
& \lambda_{1}+\cdots+\lambda_{i} \geq \mu_{1}+\cdots+\mu_{i} \text { for all } i \geq 1 .
\end{aligned}
$$

The conjugate of a partition $\lambda=\left(\lambda_{1}, \ldots, \lambda_{p}\right)$ is the partition $\lambda^{\prime}=\left(\lambda_{1}^{\prime}\right.$, $\left.\lambda_{2}^{\prime}, \ldots\right)$, where $\lambda_{i}^{\prime}=\#\left\{j \mid \lambda_{j} \geq i\right\}$. In particular, $\lambda_{1}^{\prime}=l(\lambda)$ and $\lambda_{1}=l\left(\lambda^{\prime}\right)$.

For each partition $\lambda=\left(\lambda_{1}, \lambda_{2}, \ldots, \lambda_{p}\right)$ we define

$$
n(\lambda)=\sum_{i=1}^{p}(i-1) \lambda_{i}=\sum_{1 \leq i<j \leq p} \min \left(\lambda_{i}, \lambda_{j}\right) .
$$

The concatenation $\mu * \nu$ of two compositions $\mu=\left(\mu_{1}, \mu_{2} \ldots, \mu_{r}\right)$ and $\nu=\left(\nu_{1}, \nu_{2}, \ldots, \nu_{s}\right)$ is defined to be the composition

$$
\mu * \nu=\left(\mu_{1}, \mu_{2}, \ldots, \mu_{r}, \nu_{1}, \nu_{2}, \ldots, \nu_{s}\right) .
$$

For any compositions $\mu$ and $\nu$ we define $\mu+\nu$ to be the sum of the sequences $\mu$ and $\nu$ :

$$
(\mu+\nu)_{i}=\mu_{i}+\nu_{i}
$$

Thus, for example, $n \mu=\left(n \mu_{1}, n \mu_{2}, \ldots, n \mu_{r}\right)$. 
Definition 2.1. We say that a sequence of partitions $\boldsymbol{\mu}=\left(\mu^{(1)}, \mu^{(2)}\right.$, $\left.\ldots, \mu^{(r)}\right)$ is a dominant one, if the concatenation $\mu^{(1)} * \mu^{(2)} * \cdots * \mu^{(r)}$ is a partition.

Definition 2.2. Let $\mu=\left(\mu_{1}, \mu_{2}, \ldots, \mu_{r}\right)$ and $\eta=\left(\eta_{1}, \eta_{2}, \ldots, \eta_{p}\right)$ be compositions, we say that the composition $\mu$ is compatible with $\eta$ if the all compositions

$$
\mu^{(i)}=\left(\mu_{\eta_{1}+\cdots+\eta_{i-1}+1}, \ldots, \mu_{\eta_{1}+\cdots+\eta_{i}}\right), \quad 1 \leq i \leq p
$$

appear to be partitions (possibly with zeros at the end), where by definition we put $\eta_{0}:=0$.

In other words, the composition $\mu$ is the concatenation of partitions $\mu^{(i)}$, $1 \leq i \leq p$. Conversely, if a composition $\mu$ is the concatenation of partitions $\mu^{(i)}, \quad 1 \leq i \leq p$, then the composition $\eta$ can be reconstructed from that $\mu$ as follows:

$$
\eta=\left(l l\left(\mu^{(1)}\right), l l\left(\mu^{(2)}\right), \ldots, l l\left(\mu^{(p)}\right)\right) .
$$

\section{§2.2. Kostka-Foulkes polynomials}

In Sections 2.2 till that 2.6 we will assume that all partitions which will appear, are proper.

Definition 2.3. The Kostka-Foulkes polynomials are defined as the matrix elements of the transition matrix

$$
K(q)=M(s, P)
$$

from the Schur functions $s_{\lambda}(x)$ to the Hall-Littlewood functions $P_{\mu}(x ; q)$ :

$$
s_{\lambda}(x)=\sum_{\mu} K_{\lambda \mu}(q) P_{\mu}(x ; q) .
$$

It is well known, see e.g. [53], Chapter I, that if $\lambda$ and $\mu$ are partitions, then

- $K_{\lambda \mu}(q) \neq 0$ if and only if $\lambda \geq \mu$ with respect to the dominance partial ordering " $\geq$ " on the set of partitions.

- If $\lambda \geq \mu, K_{\lambda \mu}(q)$ is a monic of degree $n(\mu)-n(\lambda)$ polynomial with non-negative integer coefficients. This result is due to A. Lascoux and M.P. Schützenberger [48]. 
- If $l(\mu)=n$, then

$$
K_{\lambda \mu}(q):=\sum_{w \in \Sigma_{n}}(-1)^{l(w)} K_{n}(w(\lambda+\delta)-\mu-\delta \mid q)
$$

where $l(w)$ denotes the length of a permutation $w \in \Sigma_{n}, \delta:=\delta_{n}=(n-1, n-$ $2, \ldots, 1,0)$, and for any $\gamma \in \mathbb{Z}^{n},|\gamma|=0, K_{n}(\gamma \mid q)$ stands for a $q$-analog of the Kostant partition function $K_{n}(\gamma)$, see e.g. [53], Chapter III, Section 6, Example 4, or Section 3 of the present paper.

Theorem 2.4. $\quad$ Let $\lambda$ and $\mu$ be partitions of the same size. There exists a polynomial $\mathcal{E}_{\lambda, \mu}(t)$ with rational coefficients such that for any integer $N \geq 1$ one has

$$
\mathcal{E}_{\lambda, \mu}(N)=K_{N \lambda, N \mu}(1)
$$

Corollary 2.5. The Ehrhart polynomial $\mathcal{E}_{\lambda, \mu}(t)$ of the Gelfand-Tsetlin polytope $G T(\lambda, \mu)$ is a polynomial, even though the polytope $G T(\lambda, \mu)$ itself does not necessary appear to be an integral one.

For a definition of the Gelfand-Tsetlin polytope see, e.g. [8, 36] or [12]. For a definition and basic properties of the Ehrhart polynomial of a convex integral polytope see, e.g. [67] or [24].

Theorem 2.4 and Corollary 2.5 are a particular case of a more general result, see Section 4, Corollary 4.15.

We refer the reader to a paper [12] which contains a rich information about vertices of Gelfand-Tsetlin's polytopes. In particular, one can find in [12] several examples of Gelfand-Tsetlin's polytopes with some non-integral vertices.

Conjecture 2.6. Let $\lambda$ and $\mu$ be (proper) partitions of the same size, then the Ehrhart polynomial $\mathcal{E}_{\lambda, \mu}(t)$ has non-negative rational coefficients.

We remark that Conjecture 2.6 is a special case of Polynomiality Conjecture from Section 6 .

Polynomiality of the function $N \longrightarrow K_{N \lambda, N \mu}(1)$ has been proved independently by several authors: W. Baldoni-Silva and M. Vergne [2], S. Billey, V. Guillemin and E. Rassart [8],...

Problem 2.7. $\quad$ Find a fermionic, i.e. a positive linear combination of products of powers of $t$ and t-binomial coefficients, formula for the polynomials $\mathcal{E}_{\lambda \mu}(t)$. 
This problem should be a very difficult one, however, since, for example, the polynomial

$$
\mathcal{E}_{\left(n^{n}\right),\left((n-1)^{n}, 1^{n}\right)}(t)
$$

coincides with the Ehrhart polynomial of the Birkhoff polytope $\mathcal{B}_{n}$ of doubly stochastic matrices, see [35], Section 7.5. We refer the reader to a paper by M. Beck and D. Pixton [3] and the literature quoted therein, for a further information about the Ehrhart polynomials (for $n \leq 9$ ) and the volumes (for $n \leq 10$ ) of the Birkhoff polytope $\mathcal{B}_{n}$.

The (normalized) leading coefficient of Ehrhart's polynomial $\mathcal{E}_{\lambda \mu}(t)$ is equal to the (normalized) volume of Gelfand-Tsetlin's polytope $G T(\lambda, \mu)$, and is known in the literature, see e.g. [23, 59], as a continuous analog of the weight multiplicity $\operatorname{dim} V_{\lambda}(\mu)$.

Finally, we would like to note that in general, the Ehrhart polynomial of a convex integral polytope may have negative coefficients. The famous example is the Reeve tetrahedron, see e.g. [35], Example 7.34, 6, and the literature quoted therein.

\section{$\S 2.3$. Skew Kostka-Foulkes polynomials}

Let $\lambda, \mu$ and $\nu$ be partitions, $\lambda \supset \mu$, and $|\lambda|=|\mu|+|\nu|$.

Definition 2.8. The skew Kostka-Foulkes polynomials $K_{\lambda \backslash \mu, \nu}(q)$ are defined as the transition coefficients from the skew Schur functions $s_{\lambda \backslash \mu}(x)$ to the Hall-Littlewood functions $P_{\nu}(x ; q)$ :

$$
s_{\lambda \backslash \mu}(x)=\sum_{\nu} K_{\lambda \backslash \mu, \nu}(q) P_{\nu}(x ; q) .
$$

It is clear that

$$
K_{\lambda \backslash \mu, \nu}(q)=\sum_{\pi} c_{\mu \pi}^{\lambda} K_{\pi \nu}(q)
$$

where the coefficients $c_{\mu \pi}^{\nu}=\operatorname{Mult}\left[V_{\nu}: V_{\mu} \otimes V_{\pi}\right]$ stand for the LittlewoodRichardson numbers.

Let us remark that

$$
K_{\lambda \backslash \mu, \nu}(q)=\sum_{T} q^{c(T)}
$$

summed over all semistandard skew tableaux $T$ of shape $\lambda \backslash \mu$ and weight $\nu$, where $c(T)$ denotes the charge of a skew tableau $T$. 
In the case $\mu=\emptyset$, the formula (2.9) is due to A. Lascoux and M.-P. Schützenberger [48]. See also [10], Chapter II, for an extended exposition of [48]. We refer the reader to [53], Chapter III, Section 6, for the definition of the Lascoux-Schützenberger statistics charge on the set of semistandard Young tableaux.

We will use also the cocharge version of the skew Kostka-Foulkes polynomials:

$$
\bar{K}_{\lambda \backslash \mu, \nu}(q)=\sum_{\pi} c_{\mu \pi}^{\lambda} \bar{K}_{\pi \mu}(q),
$$

where $\bar{K}_{\lambda \mu}(q)=q^{n(\mu)} K_{\lambda \mu}\left(q^{-1}\right)$.

( $)$ We will see in Section 5.1, example $3^{0}$, that the skew Kostka-Foulkes polynomials are some special cases of the parabolic Kostka polynomials.

\section{§2.4. Littlewood-Richardson numbers and Saturation Theorem}

The Littlewood-Richardson numbers $c_{\lambda, \mu}^{\nu}, L R$-numbers for short, are defined as the structural constants of the multiplication of Schur functions. More specifically, let $\lambda$ and $\mu$ be partitions, then

$$
s_{\lambda} s_{\mu}=\sum_{\nu} c_{\lambda, \mu}^{\nu} s_{\nu}
$$

or equivalently,

$$
s_{\nu \backslash \mu}=\sum_{\lambda} c_{\lambda, \mu}^{\nu} s_{\lambda}
$$

We have $c_{\lambda, \mu}^{\nu}=0$ unless $|\nu|=|\lambda|+|\mu|$ and $\nu \supset \lambda, \mu$. A pure combinatorial way to compute the $L R$-numbers is given by the celebrated Littlewood-Richardson rule, see e.g. [53], Chapter I, Section 9.

Saturation Theorem (A. Knutson and T. Tao [43])

$$
c_{N \lambda, N \mu}^{N \nu} \neq 0 \text { for some integer } N \geq 1 \text { if and only if } c_{\lambda, \mu}^{\nu} \neq 0 .
$$

We refer the reader to interesting and nice written papers by W. Fulton $[20,21]$ and A. Zelevinsky [74] for detailed account to an origin of Saturation Conjecture (now a theorem by A. Knutson and T. Tao) and its connections with the so-called Horn Problem.

\section{$\S 2.5$. Internal product of Schur functions, and polynomials $L_{\alpha, \beta}^{\mu}(q)$}

The irreducible characters $\chi^{\lambda}$ of the symmetric group $\Sigma_{n}$ are indexed in a natural way by partitions $\lambda$ of $n$. If $w \in \Sigma_{n}$, then define $\rho(w)$ to be the 
partition of $n$ whose parts are the cycle lengths of $w$. For any partition $\lambda$ of $m$ of length $l$, define the power-sum symmetric function

$$
p_{\lambda}=p_{\lambda_{1}} \ldots p_{\lambda_{l}}
$$

where $p_{n}(x)=\sum x_{i}^{n}$. For brevity write $p_{w}:=p_{\rho(w)}$. The Schur functions $s_{\lambda}$ and power-sums $p_{\mu}$ are related by a famous result of Frobenius

$$
s_{\lambda}=\frac{1}{n !} \sum_{w \in \Sigma_{n}} \chi^{\lambda}(w) p_{w} .
$$

For a pair of partitions $\alpha$ and $\beta,|\alpha|=|\beta|=n$, let us define the internal product $s_{\alpha} * s_{\beta}$ of Schur functions $s_{\alpha}$ and $s_{\beta}$ :

$$
s_{\alpha} * s_{\beta}=\frac{1}{n !} \sum_{w \in \Sigma_{n}} \chi^{\alpha}(w) \chi^{\beta}(w) p_{w} .
$$

It is well-known, see e.g. [53], Chapter I, Section 7, that

$$
s_{\alpha} * s_{(n)}=s_{\alpha}, \quad s_{\alpha} * s_{\left(1^{n}\right)}=s_{\alpha^{\prime}}
$$

where $\alpha^{\prime}$ denotes the conjugate partition to $\alpha$.

Let $\alpha, \beta, \gamma$ be partitions of a natural number $n \geq 1$, consider the following numbers

$$
g_{\alpha \beta \gamma}=\frac{1}{n !} \sum_{w \in \Sigma_{n}} \chi^{\alpha}(w) \chi^{\beta}(w) \chi^{\gamma}(w) .
$$

The numbers $g_{\alpha \beta \gamma}$ coincide with the structural constants for multiplication of the characters $\chi^{\alpha}$ of the symmetric group $\Sigma_{n}$ :

$$
\chi^{\alpha} \chi^{\beta}=\sum_{\gamma} g_{\alpha \beta \gamma} \chi^{\gamma}
$$

Hence, $g_{\alpha \beta \gamma}$ are non-negative integers. It is clear that

$$
s_{\alpha} * s_{\beta}=\sum_{\gamma} g_{\alpha \beta \gamma} s_{\gamma} \text {. }
$$

Remark 2.9. More generally, let $A$ and $B$ be two skew diagrams and $\gamma$ be a partition all of the same cardinality $n$. Define the coefficients $g_{A, B, \gamma}$ and the internal product $s_{A} * s_{B}$ of skew Schur functions $s_{A}$ and $s_{B}$ as follows. Let $\chi^{A}$ and $\chi^{B}$ be the characters of representations of the symmetric group $\Sigma_{n}$ 
which correspond to the skew diagrams $A$ and $B$. The numbers $g_{A, B, \gamma}$ are defined via the decomposition

$$
\chi^{A} \chi^{B}=\sum_{\gamma} g_{A, B, \gamma} \chi^{\gamma}
$$

The internal product of the skew Schur functions $s_{A}$ and $s_{B}$ is defined as follows

$$
s_{A} * s_{B}=\sum_{\gamma} g_{A, B, \gamma} s_{\gamma} .
$$

Finally, let $C$ be one more skew diagram, define the number $g_{A, B, C}$ to be equal to $\left\langle s_{A} * s_{B}, s_{C}\right\rangle$, where $\langle$,$\rangle denotes the Redfield-Hall scalar product on the$ ring of symmetric functions, see [53], Chapter I, Section 4.

Remark 2.10. It is one of the most fundamental open problems in Combinatorics and Representation Theory of the symmetric group that to find a combinatorial rule for description of the numbers $g_{\alpha \beta \gamma}$.

Theorem 2.11. Let $\alpha, \beta$ and $\gamma$ be partitions of the same size $n$.

(अ) If $g_{\alpha \beta \gamma} \neq 0$, then $g_{N \alpha, N \beta, N \gamma} \neq 0$ for any integer $N \geq 1$.

Remark 2.12. The converse statement, i.e. if $g_{N \alpha, N \beta, N \gamma} \neq 0$ for some integer $N \geq 2$, then $g_{\alpha \beta \gamma} \neq 0$, the so-called saturation property of the structural constants $g_{\alpha \beta \gamma}$, is not true in general if $n \geq 7$, even under the additional assumption that partitions $\alpha, \beta, \gamma$ and their conjugate ones $\alpha^{\prime}, \beta^{\prime}, \gamma^{\prime}$, all have at least two different parts. For example,

$g_{(6,1),\left(4,1^{3}\right),(3,3,1)}=0$, but $g_{(12,2),\left(8,2^{3}\right),(6,6,2)} \geq 1, g_{(5,2),(4,3),\left(4,1^{3}\right)}=0$, but $g_{(10,4),(8,6),\left(8,2^{3}\right)} \geq 1$,

$g_{\left(6,1^{2}\right),\left(6,1^{2}\right),(4,3,1)}=0$, but $g_{\left(12,2^{2}\right),\left(12,2^{2}\right),(8,6,2)} \geq 1, g_{(6,2),\left(6,1^{2}\right),\left(4,2^{2}\right)}=0$,

but $g_{(12,4),\left(12,2^{2}\right),\left(8,4^{2}\right)} \geq 1$.

On the other hand,

$g_{(3,1,1),(3,2),\left(2,1^{3}\right)}=1$ and $g_{(6,2,2),(6,4),\left(4,2^{3}\right)}=2, g_{(2,1),(2,1),\left(1^{3}\right)}=1$ and $g_{(4,2),(4,2),\left(2^{3}\right)}=1$,

$g_{(2,2),(2,2),(2,2)}=1$ and $g_{(4,4),(4,4),(4,4)}=1, g_{(2,2),(2,2),\left(1^{4}\right)}=1$ and $g_{(4,4),(4,4),\left(2^{4}\right)}=1$.

( However, we expect that the formal power series

$$
\sum_{N \geq 1} g_{N \alpha, N \beta, N \gamma} t^{N}
$$

is a rational function of $t$ (with the only possible pole at $t=1$ ??). 
Remark 2.13 (Plethysm structural constants).

Fix integer numbers $k$ and $n \geq 2$, and a finite dimensional representation $W$ of the Lie algebra $\mathfrak{g} l_{n}$. The $k$-th tensor power $W^{\otimes k}$ of the $\mathfrak{g} l_{n}$-module $W$ has a natural structure of $\Sigma_{k} \times \mathfrak{g} l_{n}$-module, where $\Sigma_{k}$ denotes the symmetric group of order $k$ !. Let

$$
W^{\otimes k}=\sum_{\mu, \pi} a_{\mu, W}^{\pi} S^{\mu} \otimes V_{\pi}
$$

be the decomposition of the module $W^{\otimes k}$ into irreducible $\Sigma_{k} \times \mathfrak{g} l_{n}$-submodules. Here $\mu$ is a partition of size $k$, and $S^{\mu}$ stands for the irreducible representation of the symmetric group $\Sigma_{k}$ which corresponds to the partition $\mu ; \pi$ is a partition of length at most $n$ and $V_{\pi}$ denotes the irreducible $\mathfrak{g} l_{n}$-module with the highest weight $\pi$.

If $W=V_{\lambda}$ is the irreducible $\mathfrak{g} l_{n}$-module with the highest weight $\lambda$, then the numbers $a_{\lambda, \mu}^{\pi}:=a_{\mu, V_{\lambda}}^{\pi}$ coincide with the structural constants of yet another multiplication, called plethysm, in the ring of symmetric functions $\Lambda$ :

$$
s_{\lambda} \circ s_{\mu}=\sum_{\pi} a_{\lambda, \mu}^{\pi} s_{\pi}
$$

Note, that the plethysm is an associative, but not commutative operation.

It is well-known, see e.g. [69], that if $\alpha$ and $\beta$ are partitions of the same size $k$ such that $l(\alpha)=r, l(\beta)=s$ and $n \geq r+s$, and furthermore, $W=\mathfrak{g} l_{n}$ is the adjoint representation, and

$$
\pi=(k+\alpha_{1}, \ldots, k+\alpha_{r}, \underbrace{k \ldots, k}_{n-r-s}, k-\beta_{s}, \ldots, k-\beta_{1}),
$$

then

$$
a_{\mu, \mathfrak{g} l_{n}}^{\pi}:=\left[S^{\mu} \otimes V_{\pi}: \mathfrak{g} l_{n}^{\otimes k}\right]=g_{\alpha \beta \mu} .
$$

Hence, the inner product structure constants $g_{\alpha \beta \gamma}$, and therefore the $L R$ numbers, are certain special cases of the plethysm structural constants $a_{\mu, W}^{\pi}$.

Conjecture 2.14. Let $\mu$ and $\pi, l(\pi) \leq n$, be partitions such that $\mu$ has at least two different parts. Let $W$ be a finite dimensional $\mathfrak{g} l_{n}$-module.

If $a_{\mu, W}^{\pi} \neq 0$, then $a_{N \mu, W}^{N \pi} \neq 0$, for any integer $N \geq 1$.

( Moreover, we expect that if $N_{1}$ and $N_{2}$ are integers such that $N_{1} \geq$ $N_{2}$, then 
$a_{N_{1} \mu, W}^{N_{1} \pi} \geq a_{N_{2} \mu, W}^{N_{2} \pi}$, and the formal power series

$$
\sum_{N \geq 1} a_{N \mu, W}^{N \pi} t^{N}
$$

is a rational function of $t$ (with the only possible pole at $t=1$ ??).

(a) We want to emphasize that the plethysm structural constants $a_{\mu, W}^{\pi}$ do not satisfy the so-called saturation property, i.e. it's not true, in general, that if $a_{N \mu, W}^{N \pi} \neq 0$ for some integer $N \geq 2$, then $a_{\mu, W}^{\pi} \neq 0$.

Using the tables of plethysms from [1], we have checked that

$a_{(2,2),(4,2)}^{\left(6,4^{2}, 2^{5}\right)}=1$, but $a_{(2,2),(2,1)}^{\left(3,2^{2}, 1^{5}\right)}=0, a_{(2,2),(4,2)}^{\left(4^{5}, 2^{2}\right)}=1$, but $a_{(2,2),(2,1)}^{\left(2^{5}, 1^{2}\right)}=0$.

( B) Based on several examples, we expect that if $a_{2 \mu, W}^{2 \pi} \geq 2$, then $a_{\mu, W}^{\pi} \neq 0$.

On the other hand, Conjecture 2.14 is not true if a partition $\mu$ has a form $\left(1^{k}\right)$. For example, $=1$.

$$
a_{(2,1,1),(1,1,1)}^{(4,4,2,1,1)}=1 \text {, but } a_{(2,1,1),(2,2,2)}^{(8,8,4,2,2)}=0, a_{(2,1,1),(1,1,1)}^{(4,3,3,1,1)}=0 \text {, but } a_{(2,1,1),(2,2,2)}^{(8,6,6,2,2)}
$$

Question 2.15. Could it be true that for any finite dimensional $\mathfrak{g} l_{n}$ module $W$ there exists a polynomial $p_{W}(t)\left(p_{W}(t)=t\right.$ ??) such that for all partitions $\pi$ and $\mu$ one has

$$
\text { if } a_{N \mu, W}^{N \pi} \geq p_{W}(N), \text { then } a_{\mu, W}^{\pi} \neq 0 .
$$

(\$) It is one of the most fundamental problems of Algebraic Combinatorics, Representation Theory, Theory of Invariants, ... that to find a combinatorial rule for description of the numbers $a_{\mu, W}^{\pi}$.

Definition 2.16. The polynomials $L_{\alpha \beta}^{\mu}(q)$ are defined via the decomposition of the internal product of Schur functions $s_{\alpha} * s_{\beta}(x)$ in terms of the Hall-Littlewood functions:

$$
s_{\alpha} * s_{\beta}(x)=\sum_{\mu} L_{\alpha \beta}^{\mu}(q) P_{\mu}(x ; q)
$$

In a similar fashion one can define the polynomials $L_{A, B}^{\mu}(q)$, where $A$ and $B$ are skew diagrams and $\mu$ is a partition:

$$
s_{A} * s_{B}(x)=\sum_{\mu} L_{A, B}^{\mu}(q) P_{\mu}(x ; q) .
$$




\section{Examples 2.17.}

(i) Take $n=4, \alpha=(3,1)$ and $\beta=(2,2)$.

Then the all non-zero polynomials $L_{(3,1),(2,2)}^{\mu}(q)$ are:

$$
\begin{aligned}
& L_{(3,1),(2,2)}^{(3,1)}(q)=1, L_{(3,1),(2,2)}^{(2,2)}(q)=q, L_{(3,1),(2,2)}^{(2,1,1)}(q)=1+q+q^{2}, \\
& L_{(3,1),(2,2)}^{\left(1^{4}\right)}(q)=q(1,1,2,1,1) .
\end{aligned}
$$

(ii) Take $n=6$ and $\alpha=\beta=(3,2,1)$, then

$$
\begin{aligned}
L_{\alpha, \beta}^{(6)}(q) & =1, L_{\alpha, \beta}^{(5,1)}(q)=2+q, L_{\alpha, \beta}^{(4,2)}(q)=(3,2,1), \\
L_{\alpha, \beta}^{(4,1,1)}(q) & =(4,5,2,1)=(1+q)(4,1,1), \\
L_{\alpha, \beta}^{\left(3,1^{3}\right)}(q) & =(4,9,12,11,5,2,1)=(1+q)(4,5,7,4,1,1), \\
L_{\alpha, \beta}^{\left(2,1^{4}\right)}(q) & =(1+q)^{2}\left(1+q^{2}\right)^{2}(2,3,0,1) .
\end{aligned}
$$

(iii) Take $n=6, \alpha=(4,2)$ and $\beta=(3,2,1)$, then

$$
\begin{aligned}
L_{\alpha, \beta}^{(5,1)}(q) & =1, L_{\alpha, \beta}^{(4,2)}(q)=2+q, L_{\alpha, \beta}^{(4,1,1)}(q)=(2,3,1), L_{\alpha, \beta}^{(3,3)}(q)=(1,2,1), \\
L_{\alpha, \beta}^{\left(3,1^{3}\right)}(q) & =(1+q)\left(1+q+q^{2}\right)(2,1,1), L_{\alpha, \beta}^{(2,2,1,1)}(q) \\
& =(1+q)^{2}\left(1+q+q^{2}\right)(2,0,1), \\
L_{\alpha, \beta}^{\left(2,1^{4}\right)}(q) & =(1+q)^{2}\left(1+q+q^{2}\right)\left(1+q+q^{2}\right)(1,1,0,1) .
\end{aligned}
$$

(iv) Take $n=6, \alpha=(4,2)$ and $\beta=\left(2^{3}\right)$. Then

$$
\begin{aligned}
L_{\alpha, \beta}^{(4,1,1)}(q) & =q, L_{\alpha, \beta}^{(3,3)}(q)=q, L_{\alpha, \beta}^{(3,2,1)}(q)=1+q+q^{2}, \\
L_{\alpha, \beta}^{(2,2,1,1)}(q) & =q(3,2,3,1,1), L_{\alpha, \beta}^{\left(2,1^{4}\right)}(q)=(1,1,1)(1,0,2,1,2,0,1), \\
L_{\alpha, \beta}^{\left(1^{6}\right)}(q) & =(1,0,1,1,0,1) \widetilde{K}_{\alpha,\left(^{6}\right)}(q) .
\end{aligned}
$$

Hereafter we shell use the notation $\widetilde{K}_{\alpha, \mu}(q)$ to denote the polynomial $q^{n(\mu)-n(\alpha)} K_{\alpha, \mu}\left(q^{-1}\right)$.

Remark 2.18. It is not true in general that if $\alpha, \beta, \mu$ are partition and $\alpha \geq \mu$, then the ratio $L_{\alpha, \beta}^{\mu}(1) / K_{\alpha, \mu}(1) \in \mathbb{Z}$.

For example, take $\alpha=\beta=(6,2,1)$ and $\mu=(3,3,2,1)$. Then

$$
L_{\alpha, \beta}^{\mu}(q)=(2,17,44,63,64,48,29,15,6,2,1), \widetilde{K}_{\alpha, \mu}(q)=(1,2,2,1)
$$

and $L_{\alpha, \beta}^{\mu}(1)=291, L_{\alpha, \beta}^{\mu}(-1)=1$. 
We see that $\widetilde{K}_{\alpha, \mu}(q)$ is not a divisor of $L_{\alpha, \beta}^{\mu}(q)$, and the ratio $L_{\alpha, \beta}^{\mu}(1) / K_{\alpha, \mu}$ $(1) \notin \mathbb{Z}$. Note that $L_{\alpha, \beta}^{\mu}(0)=c_{(2,1),(2,1)}^{(3,2,1)}=2$ and $\operatorname{deg} L_{\alpha, \beta}^{\mu}(q)=10=n(\mu)$ in a good agreement with Conjecture 2.23.

It follows from (2.6) and (2.16) that

$$
L_{\alpha \beta}^{\mu}(q)=\sum_{\gamma} g_{\alpha \beta \gamma} K_{\gamma \mu}(q) .
$$

Thus, the polynomials $L_{\alpha \beta}^{\mu}(q)$ have non-negative integer coefficients, and

$$
L_{\alpha \beta}^{\mu}(0)=g_{\alpha \beta \mu} .
$$

It follows from (2.18) that the number $L_{\alpha, \beta}^{\mu}(1)$ is equal to $\left\langle s_{\alpha} * s_{\beta}, h_{\mu}\right\rangle$, where $\langle$,$\rangle denotes the Redfield-Hall scalar product on the ring of symmetric$ functions, see [53]. In other words,

$$
s_{\alpha}(x) * s_{\beta}(x)=\sum_{\mu} L_{\alpha, \beta}^{\mu}(1) m_{\mu}(x),
$$

where $m_{\mu}(x)$ denotes the monomial symmetric function corresponding to partition $\mu$. Therefore, the numbers $L_{\alpha, \beta}^{\mu}(1)$ and $L_{A, B}^{\mu}(1)$ can be defined for any composition $\mu$.

Remark 2.19. There is a well-known connection between the structural constants $g_{\alpha \beta \gamma}$ and the numbers $L_{\alpha, \beta}^{\mu}(1)$. Namely, let $A, B$ and $C=\Gamma \backslash \gamma$ be skew diagrams such that the partition $\Gamma$ has the length at most $n$, and $|A|=|B|=|C|$. Then

$$
g_{A, B, C}=\sum_{w \in \Sigma_{n}}(-1)^{l(w)} L_{A, B}^{w \circ C}(1),
$$

where $w \circ C$ stands for the composition $w\left(\Gamma+\delta_{n}\right)-\gamma-\delta_{n}$, and $\delta_{n}=(n-1, n-$ $2, \ldots, 1,0)$.

The polynomials $L_{\alpha \beta}^{\mu}(q)$ can be considered as a generalization of the Kostka-Foulkes polynomials. Indeed, if partition $\beta$ consists of one part, $\beta=$ $(n)$, then

$$
L_{\alpha \beta}^{\mu}(q)=K_{\alpha, \mu}(q), \quad L_{\alpha \beta^{\prime}}^{\mu}(q)=K_{\alpha^{\prime}, \mu}(q) .
$$

Proposition 2.20. Let $\alpha, \beta$ and $\mu=\left(\mu_{1} \geq \cdots \geq \mu_{r}\right)$ be partitions of the same size $n$. Then

$$
L_{\alpha, \beta}^{\mu}(1)=\sum_{\boldsymbol{\mu}} K_{\alpha, \boldsymbol{\mu}}(1) K_{\beta, \boldsymbol{\mu}}(1),
$$


where the sum runs over sequences of partitions $\boldsymbol{\mu}=\left(\mu^{(1)}, \ldots, \mu^{(r)}\right)$ such that $\left|\mu^{(a)}\right|=\mu_{a}, 1 \leq a \leq r$.

Corollary 2.21. If $\mu=\left(r, 1^{s}\right)$ is a hook partition, then

$$
L_{\alpha, \beta}^{\mu}(1)=\sum_{|\lambda|=r} K_{\alpha \backslash \lambda,\left(1^{s}\right)}(1) K_{\beta \backslash \lambda,\left(1^{s}\right)}(1)
$$

where the sum runs over all partitions $\lambda$ of size $r, \lambda \subset \alpha \cap \beta$.

In particular, $L_{\alpha \beta}^{\left(1^{n}\right)}(1)=f^{\alpha} f^{\beta}$, where $f^{\alpha}$ denotes the number of standard Young tableaux of shape $\alpha$. More generally [35],

$$
L_{\alpha \beta}^{\left(1^{n}\right)}(q)=K_{\beta^{\prime} \alpha}(q, q) \widetilde{K}_{\alpha,\left(1^{n}\right)}(q)=K_{\alpha^{\prime} \beta}(q, q) \widetilde{K}_{\beta,\left(1^{n}\right)}(q),
$$

where

$$
\widetilde{K}_{\alpha, \beta}(q):=q^{n(\beta)-n(\alpha)} K_{\alpha \beta}\left(q^{-1}\right), \quad K_{\alpha \beta}(q, q):=\left.K_{\alpha \beta}(q, t)\right|_{t=q},
$$

and $K_{\alpha \beta}(q, t)$ stands for the double Kostka polynomial introduced by I. Macdonald [53], Chapter VI, (8.11).

Problem 2.22. Find a q-analog of the equality (2.21).

Conjecture 2.23 (Saturation conjecture for polynomials $L_{\alpha, \beta}^{\mu}(q)$ ). Let $\alpha, \beta$ and $\mu$ be partitions of the same size such that $L_{\alpha, \beta}^{\mu}(q) \neq 0$. Then $(\triangleleft)$ For any integer $N \geq 1$,

- $\max \operatorname{deg} L_{N \alpha, N \beta}^{N \mu}(q)=N \max \operatorname{deg} L_{\alpha, \beta}^{\mu}(q)$;

- If partition $\mu$ either has at least two different parts, or $\mu$ has a rectangular shape, but $\mu$ is different from the both partitions $\alpha$ and $\beta$, and their conjugate ones $\alpha^{\prime}$ and $\beta^{\prime}$, then

$\min \operatorname{deg} L_{N \alpha, N \beta}^{N \mu}(q)=N \min \operatorname{deg} L_{\alpha, \beta}^{\mu}(q)$.

$(\downarrow) \max \operatorname{deg} L_{\alpha, \beta}^{\mu}(q)=n(\mu)-A(\alpha, \beta)$, where $A(\alpha, \beta)$ stands for the min $\operatorname{deg} K_{\alpha, \beta}(q, q)$, i.e.

$K_{\alpha, \beta}(q, q)=B(\alpha, \beta) q^{A(\alpha, \beta)}+$ higher degree terms.

$(\triangleleft \diamond)$ ) (Saturation conjecture for polynomials $K_{\alpha, \beta}(q, q)$ )

For any integer $N \geq 1, \quad A(N \alpha, N \beta)=N A(\alpha, \beta)$. 
Examples 2.24. $\quad$ (i) Take $n=3$,

$$
\begin{aligned}
& L_{(2,1),(2,1)}^{(3)}(q)=1, \quad L_{(4,2),(4,2)}^{(6)}(q)=1, \\
& L_{(2,1),(2,1)}^{\left(1^{3}\right)}(q)=1+q+q^{2}+q^{3}, \\
& L_{(4,2),(4,2)}^{\left(2^{3}\right)}(q)=1+2 q+4 q^{2}+3 q^{3}+3 q^{4}+q^{5}+q^{6}, \\
& L_{(2,1),(2,1)}^{(2,1)}(q)=1+q, \quad L_{(4,2),(4,2)}^{(4,2)}(q)=2+q+q^{2} . \\
& L_{(2,1),\left(1^{3}\right)}^{(2,1)}(q)=1, \quad L_{(4,2),\left(2^{3}\right)}^{(4,2)}(q)=1, \\
& L_{(2,1),\left(1^{3}\right)}^{\left(1^{3}\right)}(q)=q+q^{2}, \quad L_{(4,2),\left(2^{3}\right)}^{\left(2^{3}\right)}(q)=(1,1,2,1,1) .
\end{aligned}
$$

(ii) Take $n=4$,

$$
\begin{aligned}
& L_{(3,1),(2,2)}^{(2,2)}(q)=q, \quad L_{(6,2),(4,4)}^{(4,4)}(q)=1+q^{2}, \\
& L_{(3,1),(2,2)}^{(2,1,1)}(q)=1+q+q^{2}, \quad L_{(6,2),(4,4)}^{(4,2,2)}(q)=(1,2,3,1,1), \\
& L_{(3,1),(2,2)}^{\left(1^{4}\right)}(q)=q(1,1,2,1,1), \quad L_{(6,2),(4,4)}^{\left(2^{4}\right)}(q)=q^{2}(2,2,6,5,7,4,4,1,1) .
\end{aligned}
$$

The latter example shows that for the numbers $g_{\alpha \beta \gamma}$ an obvious generalization of the Fulton conjecture, see Section 6 , is false.

Conjecture 2.25 (Rationality conjecture for polynomials $L_{\alpha, \beta}^{\mu}(q)$ ). Let $\alpha, \beta$ and $\mu$ be partitions of the same size. The generating function

$$
\sum_{N \geq 0} L_{N \alpha, N \beta}^{N \mu}(q) t^{N}
$$

is a rational function of $q$ and $t$.

Problem 2.26. Give a combinatorial interpretation of the integer numbers $L_{\alpha, \beta}^{\mu}(-1)$.

Problem 2.27. Find a fermionic type formula for the polynomials $L_{\alpha \beta}^{(\mu)}(q)$ which extends that for the Kostka-Foulkes polynomials, see Section 5.1, Theorem 5.3. 


\section{§2.6. Extended and restricted Littlewood-Richardson numbers}

\section{$\left(1^{0}\right)$ (Extended Littlewood-Richardson numbers)}

Let $\lambda, \mu$ and $\nu$ be partitions such that $|\lambda|+|\mu| \geq|\nu|$. Choose an integer number $N$ such that $N \geq N_{0}:=\max \left(|\lambda|+\lambda_{1},|\mu|+\mu_{1},|\nu|+\nu_{1}\right)$, and consider partitions

$$
\alpha_{N}:=(N-|\lambda|, \lambda), \beta_{N}:=(N-|\mu|, \mu), \gamma_{N}:=(N-|\nu|, \nu) .
$$

It is clear that $\left|\alpha_{N}\right|=\left|\beta_{N}\right|=\left|\gamma_{N}\right|=N$.

According to results by F. Murnaghan [57], Y. Dvir [17] and E. Vallejo [70], if $N \geq N_{0}$, then the number $g_{\lambda_{N}, \beta_{N}, \gamma_{N}}$ does not depend on $N$.

Definition 2.28. The extended Littlewood-Richardson number $C_{\lambda, \mu}^{\nu}$ is defined to be equal to the stable value of the numbers $g_{\lambda_{N}, \beta_{N}, \gamma_{N}}$.

More generally, the following statement is true:

Proposition 2.29. The sequence of polynomials $\left\{L_{\alpha_{N}, \beta_{N}}^{\gamma_{N}}(q)\right\}_{N \geq 1}$ is stabilized to the polynomial $\mathcal{L}_{\lambda, \mu}^{\nu}(q)$, i.e. if integer $N$ is big enough, then the polynomial $L_{\alpha_{N}, \beta_{N}}^{\gamma_{N}}(q)$ does not depend on $N$ and equal to $\mathcal{L}_{\lambda, \mu}^{\nu}(q)$. The latter is a polynomial with non-negative integer coefficients, and $\mathcal{L}_{\lambda, \mu}^{\nu}(0)=C_{\lambda, \mu}^{\nu}$.

According to another result by Y. Dvir [17], the numbers $C_{\lambda, \mu}^{\nu}$ can be considered as a generalization of the $L R$-numbers $c_{\lambda, \mu}^{\nu}$.

Proposition 2.30 (Y. Dvir [17]). If $|\lambda|+|\mu|=|\nu|$, then the number $C_{\lambda, \mu}^{\nu}$ coincides with the Littlewood-Richardson number $c_{\lambda, \mu}^{\nu}$.

Examples 2.31. (i) Take $\lambda=\mu=(2,1)$, then

$$
\begin{aligned}
C_{\lambda, \mu}^{(3,2,1)} & =c_{\lambda, \mu}^{(3,2,1)}=2, \\
C_{\lambda, \mu}^{(3,1,1)} & =6, C_{\lambda, \mu}^{(2,2,1)}=5, C_{\lambda, \mu}^{(2,1,1,1)}=4, C_{\lambda, \mu}^{(3,2)}=5, \\
C_{\lambda, \mu}^{(2,2)} & =6, C_{\lambda, \mu}^{(3,1)}=C_{\lambda, \mu}^{(2,1,1)}=9, C_{\lambda, \mu}^{(2,1)}=9 .
\end{aligned}
$$

(ii) Take $\lambda=(2,1)$ and $\mu=(3,1)$, then

$$
C_{\lambda, \mu}^{(3,1)}=13, C_{\lambda, \mu}^{(2,1)}=9 .
$$

Problem 2.32. $\quad$ Find a combinatorial rule for calculating the extended LR-numbers $C_{\lambda, \mu}^{\nu}$ which extends the Littlewood-Richardson rule. 
Conjecture 2.33 (Saturation conjecture for extended $L R$ numbers).

$C_{N \lambda, N \mu}^{N \nu} \neq 0$ for some integer $N \geq 1$ if and only if $C_{\lambda, \mu}^{\nu} \neq 0$.

$\left(2^{0}\right)$ (Restricted Littlewood-Richardson numbers, cf. [26], Exercise 13.35)

Fix positive integers $l$ and $n \geq 2$. Denote by $\Sigma_{n, l}$ the affine reflection group on $\mathbb{R}^{n}$ generated by the reflection

$$
s_{0}=\left(x_{n}+l, x_{2}, \ldots, x_{n-1}, x_{1}-l\right)
$$

and the symmetric group $\Sigma_{n}$.

Definition 2.34 (Restricted Littlewood-Richardson numbers).

Let $\lambda, \mu$ and $\nu$ be partitions such that $|\lambda|+|\mu|=|\nu|$. Define the level $l$ restricted Littlewood-Richardson number $c_{\lambda, \mu}^{\nu}[l]$ as follows

$$
c_{\lambda, \mu}^{\nu}[l]=\sum_{w \in \Sigma_{n, l}}(-1)^{l(w)} c_{\lambda, \mu}^{w \circ \nu}
$$

where $w \circ \nu$ denotes the composition $w\left(\nu+\delta_{n}\right)-\delta_{n}$, and $\delta_{n}=(n-1, \ldots, 1,0)$.

It is well-known that

$$
0 \leq c_{\lambda, \mu}^{\nu}[1] \leq c_{\lambda, \mu}^{\nu}[2] \leq \cdots=c_{\lambda, \mu}^{\nu}
$$

In a similar fashion one can define the level $l$ extended Littlewood-Richardson numbers $C_{\lambda, \mu}^{\nu}[l]$.

Conjecture 2.35 (Saturation conjecture for the level $l$ extended $L R$-numbers).

Let $\lambda, \mu$ and $\nu$ be partitions such that $|\lambda|+|\mu| \geq|\nu|$. Then

$C_{N \lambda, N \mu}^{N \nu}[l] \neq 0$ for some integer $N \geq 1$ if and only if $C_{\lambda, \mu}^{\nu}[l] \neq 0$.

Conjecture 2.36 (Polynomiality conjecture for level $l$ extended $L R$-numbers).

Let $\lambda, \mu$ and $\nu$ be partitions such that $|\lambda|+|\mu| \geq|\nu|$. Then

$C_{N \lambda, N \mu}^{N \nu}[l]$ is a polynomial in $N$ with non-negative rational coefficients.

(\$) Moreover, the formal power series

$$
\sum_{N \geq 0} C_{N \lambda, N \mu}^{N \nu}[l] t^{N}
$$


is a rational function in $t$ of the form

$$
P_{\lambda, \mu}^{\nu, l}(t) /(1-t)^{r(\lambda, \mu, \nu, l)+1}, \quad P_{\lambda, \mu}^{\nu, l}(0)=1, \quad P_{\lambda, \mu}^{\nu, l}(1) \neq 0,
$$

where $r(\lambda, \mu, \nu, l) \in \mathbb{Z}_{\geq 0}$ and $P_{\lambda, \mu}^{\nu, l}(t)$ is a polynomial with non-negative integer coefficients.

\section{$\S 3 . \quad$ Parabolic Kostant Partition Function and its $q$-analog}

\section{§3.1. Definitions: algebraic and combinatorial}

Let $\eta=\left(\eta_{1}, \eta_{2}, \ldots, \eta_{p}\right)$ be a composition, $\eta_{p}>0,|\eta|=n$. Denote by $\Phi(\eta)$ the set of ordered pairs $(i, j) \in \mathbb{Z}^{2}$ such that

$$
1 \leq i \leq \eta_{1}+\cdots+\eta_{r}<j \leq n
$$

for some $r, 1 \leq r \leq p$. For example, if $\eta=\left(1^{n}\right)$, then

$$
\Phi(\eta)=\left\{(i, j) \in \mathbb{Z}^{2} \mid 1 \leq i<j \leq n\right\} .
$$

Definition 3.1. Let $\gamma=\left(\gamma_{1}, \gamma_{2}, \ldots, \gamma_{n}\right) \in \mathbb{Z}^{n}$ be a sequence of integers such that $|\gamma|=0$, define a parabolic $q$-Kostant partition function $K_{\Phi(\eta)}(\gamma \mid q)$ via the decomposition

$$
\prod_{(i, j) \in \Phi(\eta)}\left(1-q x_{i} / x_{j}\right)^{-1}=\sum_{\gamma} K_{\Phi(\eta)}(\gamma \mid q) x^{\gamma},
$$

where the sum runs over the all sequences $\gamma=\left(\gamma_{1}, \gamma_{2}, \ldots, \gamma_{n}\right) \in \mathbb{Z}^{n}$ such that $|\gamma|=0$.

Definition 3.2. Let $K_{\Phi(\eta)}(\gamma)$ denote the parabolic Kostant partition function, that is to say, the value of the polynomial $K_{\Phi(\eta)}(\gamma \mid q)$ at $q:=1$.

Remark 3.3 (Combinatorial definition of $q$-Kostant partitionfunction).

One can give an equivalent pure combinatorial definition of the parabolic $q$-Kostant partition function $K_{\Phi(\eta)}(\gamma \mid q)$ as follows.

Let $\eta$ be a composition, $|\eta|=n$. Denote by $S M_{\eta}(\gamma)$ the set of all skewsymmetric integer matrices $M=\left(m_{i, j}\right)_{1 \leq i, j \leq n}$ such that

(i) $m_{i, j} \geq 0$, if $1 \leq i \leq j \leq n$;

(ii) $m_{i, j}=0$, if $r_{k-1}<i \leq j \leq r_{k}$ for some $k, 1 \leq k \leq p$, where $r_{k}:=\sum_{j \leq k} \eta_{j}$, and $r_{0}:=0$;

(iii) $\sum_{j=1}^{n} m_{i, j}=\gamma_{i}$, for all $i, 1 \leq i \leq n$. 
For each $M \in S M_{\eta}(\gamma)$ we define the magnitude of $M$, denoted by $\|M\|$, to be the sum $\sum_{1 \leq i \leq j \leq n} m_{i, j}$. Then

$$
K_{\Phi(\eta)}(\gamma \mid q)=\sum q^{\|M\|}
$$

where the sum runs over all matrices $M \in S M_{\eta}(\gamma)$.

Therefore, $K_{\Phi(\eta)}(\gamma)=$ Card $\left|S M_{\eta}(\gamma)\right|$.

Remark 3.4 (Generalized $q$-Kostant partition function [68]).

Let $\Sigma \subseteq \Phi\left(1^{n}\right)$ be a subset, following [68] one can define the generalized Kostant partition function $K_{\Sigma}(\gamma)$ and its $q$-analog $K_{\Sigma}(\gamma \mid q)$, from the decomposition

$$
\prod_{(i, j) \in \Sigma}\left(1-q x_{i} / x_{j}\right)^{-1}=\sum_{\gamma} K_{\Sigma}(\gamma \mid q) x^{\gamma}
$$

where the sum runs over all sequences $\gamma \in \mathbb{Z}^{n}$ such that $|\gamma|=0$. Moreover, by definition, $K_{\Sigma}(\gamma)=\left.K_{\Sigma}(\gamma \mid q)\right|_{q=1}$.

Equivalently,

$$
K_{\Sigma}(\gamma \mid q)=\sum_{M} q^{\|M\|}
$$

where the sum runs over the set of $n$ by $n$ skew-symmetric matrices $M=\left(m_{i, j}\right)$ such that

(i) $m_{i, j} \geq 0$ if $1<i \leq j \leq n$,

(ii) $m_{i, j}=0$ if $(i, j) \notin \Sigma$,

(iii) $\sum_{j} m_{i, j}=\gamma_{i}$ for all $\mathrm{i}, 1 \leq i \leq n$.

(\$) Most of our results about the parabolic $q$-Kostant partition function $K_{\Phi(\eta)}(\gamma \mid q)$, including, for example, Theorems 3.17, 3.20, 3.23, 3.25, 3.30 and 3.31 , with a small modifications, are still valid for the function $K_{\Sigma}(\gamma \mid q)$. Since we don't use the generalized Kostant partition function in the present paper, we leave this interesting subject for subsequent publications.

\section{§3.2. Elementary properties, and explicit formulas for $l(\eta) \leq 4$}

Using the above combinatorial definition of the function $K_{\Phi(\eta)}(\gamma \mid q)$, one can describe some elementary, but useful, properties of the latter.

Proposition 3.5. (i) Let $\eta_{i}, i=1,2$, be two compositions and $\gamma_{i} \in Y_{\eta_{i}}$, $i=1,2$, then

$$
K_{\Phi\left(\eta_{1} * \eta_{2}\right)}\left(\gamma_{1} * \gamma_{2} \mid q\right)=K_{\Phi\left(\eta_{1}\right)}\left(\gamma_{1} \mid q\right) K_{\Phi\left(\eta_{2}\right)}\left(\gamma_{2} \mid q\right) .
$$


(ii) Let $\eta$ be a composition and $\gamma \in Y_{\eta}$, then

$$
K_{\Phi(\eta)}(\gamma \mid q)=K_{\Phi(\overleftarrow{\eta})}(-\overleftarrow{\gamma} \mid q)
$$

where for any composition $\beta=\left(\beta_{1}, \ldots, \beta_{r-1}, \beta_{r}\right)$ the symbol $\overleftarrow{\beta}$ stands for the composition $\left(\beta_{r}, \beta_{r-1}, \ldots, \beta_{1}\right)$.

(iii) Let $\eta_{1}$ and $\eta_{2}$ be compositions such that $\eta_{2}$ is a subdivision of $\eta_{1}$, so that $\eta_{1} \geq \eta_{2}$. Then

$$
K_{\Phi\left(\eta_{1}\right)}(q) \leq K_{\Phi\left(\eta_{2}\right)}(q)
$$

See Section 1, Notation, for the definition when a composition $\eta_{2}$ is a subdivision of that $\eta_{1}$. We remark that the last statement is false if one assumes only that $\eta_{1} \geq \eta_{2}$ with respect to the dominance partial ordering on the set of compositions, see example below.

Example 3.6. Take $\gamma=(3,0,-1,-1,0,-1)$, then $(2,3,1) \geq(2,2,2)$, but

$$
K_{\Phi(2,2,2)}(\gamma \mid q)=q^{3}+2 q^{4} \leq K_{\Phi(2,3,1)}(\gamma \mid q)=q^{3}+3 q^{4} .
$$

On the other hand, $K_{\Phi(2,1,1,2)}(\gamma \mid q)=q^{3}(1,3,2,1) \geq K_{\Phi(2,2,2)}(\gamma \mid q)$.

Proposition 3.7 (Recurrence relation for parabolic $q$-Kostant partition function).

Let $\eta=\left(\eta_{1}, \ldots, \eta_{p}\right)$ be a composition of size $n, \gamma \in Y_{\eta}$. Define $\widetilde{\eta}=$ $\left(\eta_{1}, \ldots, \eta_{r_{p-1}}\right)$. Then

$$
K_{\Phi(\eta)}(\gamma \mid q)=q^{-\gamma_{n}} \sum_{\beta} K_{\Phi(\widetilde{\eta})}\left(\gamma_{1}-\beta_{1}, \ldots, \gamma_{r_{p-1}}-\beta_{r_{p-1}} \mid q\right),
$$

where the sum runs over $\beta \in \mathbb{Z}_{\geq 0}^{r_{p-1}}$ such that $|\beta|=-\gamma_{n}$.

The next proposition describes several particular cases of Theorem 3.31 below, namely, the cases when a parabolic $q$-Kostant partition function admits an explicit simple expression.

Proposition 3.8 (Explicit formulas for $l(\eta) \leq 4$ ).

(i) Let $\eta=\left(\eta_{1}, \eta_{2}\right)$ be a two component composition and $\gamma \in Y_{\eta}$. Let us introduce integer vectors $\lambda=\left(\gamma_{1}, \ldots, \gamma_{\eta_{1}}\right)$ and $\mu=\left(-\gamma_{\eta_{1}+1}, \ldots,-\gamma_{\eta_{1}+\eta_{2}}\right)$. Then $\lambda$ and $\mu$ are compositions of the same size, and

$$
K_{\Phi(\eta)}(\gamma \mid q)=\left|\mathcal{P}_{\lambda \mu}\right| q^{|\lambda|}
$$


where $\mathcal{P}_{\lambda \mu}$ denotes the set of transportation matrices of type $(\lambda ; \mu)$, i.e. the set of $l(\lambda)$ by $l(\mu)$ matrices with non-negative integer entries, and the row sums $\lambda_{i}$, and the column sums $\mu_{j}$.

(ii) Let $\eta=\left(1^{3}\right)$ and $\gamma \in Y_{\left(1^{3}\right)}$, i.e. $\gamma_{1} \geq 0$ and $\gamma_{1}+\gamma_{2} \geq 0$. Then

$$
K_{\left(1^{3}\right)}(\gamma \mid q)=q^{\max \left(\gamma_{1}, \gamma_{1}+\gamma_{2}\right)}\left[\begin{array}{c}
\min \left(\gamma_{1}, \gamma_{1}+\gamma_{2}\right) \\
1
\end{array}\right]_{q} .
$$

(iii) Let $\eta=\left(\eta_{1}, \eta_{2}, \eta_{3}\right)$ be a three component composition of size $n$, and $\gamma \in Y_{\eta}^{+}$ belongs to the dominant chamber. Then

$$
K_{\Phi(\eta)}(\gamma \mid q)=q^{-\gamma_{n}} \prod_{j=1}^{\eta_{1}} B_{q}\left(\gamma_{j}+\eta_{2} ; \eta_{2}\right)
$$

where for $n \geq k$

$$
B_{q}(n ; k)=\sum_{j=0}^{n-k}\left(\begin{array}{c}
\mathrm{J}+k-1 \\
\mathrm{~J}
\end{array}\right) q^{j}=1 /(k-1) !(\partial / \partial q)^{k-1}\left[\left(q^{k-1}-q^{n}\right) /(1-q)\right] .
$$

(iv) Let $\eta=\left(1, \eta_{2}, \eta_{3}, \eta_{4}\right)$ be a four component composition of size $n, \eta_{1}=$ 1 , and $\gamma \in Y_{\eta}^{+}$belongs to the dominant chamber. Then

$$
K_{\Phi(\eta)}(\gamma \mid q)=q^{-\gamma_{n}} \sum_{\beta} B_{q}\left(\beta_{1}+\eta_{3} ; \eta_{3}\right) \prod_{j=2}^{\eta_{2}+1} B_{q}\left(\beta_{j}+\gamma_{j}+\eta_{3} ; \eta_{3}\right) q^{\beta_{j}}
$$

where the sum runs over all vectors $\beta \in \mathbb{Z}_{\geq 0}^{\eta_{2}+1}$ such that $|\beta|=\gamma_{1}$.

In particular, if $\left(\gamma_{1}, \gamma_{2}, \gamma_{3}, \gamma_{4}\right) \in Y_{\left(1^{4}\right)}^{+}$, i.e. $\gamma_{1} \geq 0, \gamma_{2} \geq 0$ and $\gamma_{3} \geq 0$, then

$$
\begin{aligned}
& K_{\Phi\left(1^{4}\right)}\left(\gamma_{1}, \gamma_{2}, \gamma_{3}, \gamma_{4}\right) \\
& =q^{-\gamma_{4}}\left\{q\left[\begin{array}{c}
\gamma_{1}+2 \\
2
\end{array}\right]_{q}\left[\begin{array}{c}
\gamma_{2} \\
1
\end{array}\right]_{q}+\sum_{2 j \leq \gamma_{1}} q^{2 j}\left(\left[\begin{array}{c}
\gamma_{1}+1-2 j \\
1
\end{array}\right]_{q}\right)^{2}\right\} \\
& =q^{-\gamma_{n}} \sum_{j=1}^{\gamma_{1}} q^{j}\left[\begin{array}{c}
\gamma_{1}+1-j \\
1
\end{array}\right]_{q}\left[\begin{array}{c}
\gamma_{2}+1-j \\
1
\end{array}\right]_{q} .
\end{aligned}
$$

Therefore, if $\left(\gamma_{1}, \gamma_{2}, \gamma_{3}, \gamma_{4}\right) \in Y_{\left(1^{4}\right)}^{+}$, then $K_{\Phi\left(1^{4}\right)}\left(\gamma_{1}, \gamma_{2}, \gamma_{3}, \gamma_{4}\right)=$ $\left(\begin{array}{c}\gamma_{1}+3 \\ 3\end{array}\right)+\gamma_{2}\left(\begin{array}{c}\gamma_{1}+2 \\ 2\end{array}\right)$.

We remark that $\left.B_{q}(n ; l)\right|_{q=1}=\left(\begin{array}{l}n \\ l\end{array}\right)$. 
Remark 3.9. It is well-known, see e.g. [67], [13] and the literature quoted therein, that on the set of transportation matrices of size $n$ by $m$, the function $\left|\mathcal{P}_{\lambda \mu}\right|$ is a continuous piecewise polynomial function in $\lambda_{1}, \ldots$, $\lambda_{n}, \mu_{1}, \ldots, \mu_{m}$ of degree $(n-1)(m-1)$.

Question 3.10. It follows from the above Proposition and the formula (5.41) from Section 5.1, that if $N$ is big enough integer such that $\nu_{N}:=\gamma+$ $N \delta_{\eta_{1}+\eta_{2}}$ is a partition, and if we put by definition $\lambda_{N}:=N \delta_{\eta_{2}}$ and $\mu_{N}:=$ $N\left(\delta_{\eta_{1}}+(\underbrace{\eta_{2}, \ldots, \eta_{2}}_{\eta_{1}})\right)$, then

$$
\left|\mathcal{P}_{\lambda \mu}\right|=c_{\lambda_{N}, \mu_{N}}^{\nu_{N}}
$$

(\$) Is it true that if $N$ is a big enough integer, then

$$
\mathcal{P}_{\lambda \mu}(q) \doteq c_{\lambda_{N}, \mu_{N}}^{\nu_{N}}(q)
$$

where $c_{\lambda, \mu}^{\nu}(q)$ denotes the $q$-analog of the LR-numbers, introduced C. Carre and B. Leclerc, and A. Lascoux, B. Leclerc and J.-Y. Thibon, see e.g. [47]?

For the definition of polynomials $\mathcal{P}_{\lambda, \mu}(q)$ see Section 5.4, (5.48).

\section{§3.3. Non-vanishing, Degree and Saturation theorems}

It is clear from the very definition that $K_{\Phi(\eta)}(\gamma \mid q)$ is a polynomial in $q$ with non-negative integer coefficients. For example, if $\eta=\left(1^{n}\right)$, the function $K_{\Phi\left(1^{n}\right)}(\gamma \mid q)$ coincides with the $q$-analog $K_{n}(\gamma \mid q)$ of the Kostant partition function $K_{n}(\gamma)$, see e.g [2]. It is not difficult to see [35] that

$$
K_{n}(\gamma \mid q) \neq 0 \text { if and only if } \gamma \in Y_{n} \text {, where }
$$

$$
Y_{n}:=\left\{\left(\gamma_{1}, \ldots, \gamma_{n}\right) \in \mathbb{Z}^{n} \mid \sum_{i=1}^{k} \gamma_{i} \geq 0,1 \leq k \leq n, \sum_{i=1}^{n} \gamma_{i}=0\right\} .
$$

Our next goal is to generalize this result to the case of the parabolic $q$ Kostant partition function $K_{\Phi(\eta)}(\gamma \mid q)$ corresponding to an arbitrary composition $\eta$.

Definition 3.11. Let $\eta=\left(\eta_{1}, \ldots, \eta_{p}\right)$ be a composition of size $n$, denote by $Y_{\eta}$ the set of sequences $\left(\gamma_{1}, \ldots, \gamma_{n}\right) \in \mathbb{Z}^{n},|\gamma|=0$, such that for each integer $k, 0 \leq k \leq p-1$, the following inequalities are valid:

$$
\sum_{j=1}^{r_{k}} \gamma_{j}+\sum_{a \in \Omega_{k}} \gamma_{a} \geq 0 \quad \text { for all subsets } \Omega_{k} \subseteq\left[\eta_{k}+1, \ldots, \eta_{k}+\eta_{k+1}\right],
$$

where $r_{k}:=\sum_{j \leq k} \eta_{j}$, if $k \geq 1$, and $r_{0}:=0$; by definition, we put $\eta_{0}:=0$. 
In particular, we have $\gamma_{1} \geq 0, \ldots, \gamma_{\eta_{1}} \geq 0$, and $\gamma_{r_{p-1}+1} \leq 0, \ldots, \gamma_{n} \leq 0$.

Definition 3.12. Denote by $Y_{\eta}^{+}$the dominant chamber in the set $Y_{\eta}$, i.e. the subset of $Y_{\eta}$ consisting of all vectors $\gamma=\left(\gamma_{1}, \ldots, \gamma_{n}\right)$ such that $\gamma_{1} \geq$ $\cdots \geq \gamma_{n-1} \geq 0$.

(\$) We want to stress that if $\gamma \in Y_{\eta}^{+}$, then $\gamma_{r_{p-1}+1}=\cdots=\gamma_{n-1}=0$, and $\gamma_{n} \leq 0$.

Theorem 3.13 (Non-vanishing and Degree Theorem for parabolic $q$-Kostant partition function).

Let $\eta=\left(\eta_{1}, \ldots, \eta_{p}\right)$ be a composition of size $n$, and $\gamma \in \mathbb{Z}^{n}$ such that $|\gamma|=0$. Then

$$
K_{\Phi(\eta)}(\gamma \mid q) \neq 0 \text {, if and only if } \gamma \in Y_{\eta} \text {. Moreover }
$$

$$
\operatorname{deg} K_{\Phi(\eta)}(\gamma \mid q)=\sum_{k=1}^{p-1}(p-k)\left(\sum_{j=r_{k-1}+1}^{r_{k}} \gamma_{j}\right) .
$$

Remember that $r_{k}=\sum_{j \leq k} \eta_{j}$ if $k \geq 1$, and $r_{0}=0$.

Example 3.14. Take $\gamma=(2,1,0,-1,0,-1,-1)$ and $\eta=(1,2,2,1,1)$. Using formula (3.31), let us compute the degree of the parabolic $q$-Kostant partition function $K_{\Phi(\eta)}(\gamma \mid q)$. Namely,

$\operatorname{deg} K_{\Phi(\eta)}(\gamma \mid q)=2+(2+1)+(2+1-1)+(2+1-1-1)=8$. In fact, $K_{\Phi(\eta)}(\gamma \mid q)=q^{3}(3,21,52,65,42,13)$.

If $\gamma \in Y_{\eta}$, so that $K_{\Phi(\eta)}(\gamma \mid q) \neq 0$, we denote by $r(\gamma, \eta) q^{s(\gamma, \eta)}$ its leading term. For example,

$$
\begin{gathered}
r\left(\gamma,\left(1^{n}\right)\right)=1, s\left(\gamma,\left(1^{n}\right)\right)=\sum_{i=1}^{n-1}(n-i) \gamma_{i}, \\
r((3,0,-1,-1,0,-1),(2,3,1))=3, s((3,0,-1,-1,0,-1),(2,3,1))=4 .
\end{gathered}
$$

In general, the number $r(\gamma, \eta)$ can be equal to any positive integer. As for the number $s(\gamma, \eta)$, it follows from Theorem 3.15 that $s(\gamma, \eta)=\left(\gamma, \delta_{\Phi(\eta)}\right)$, where $\delta_{\Phi(\eta)}$ denotes the vector with components $\left(\delta_{\Phi(\eta)}\right)_{i}=p-k$ if $r_{k-1}<i \leq$ $r_{k}, k=1, \ldots, p$.

Moreover, the numbers $s(\gamma, \eta)$ satisfy the so-called saturation property.

Corollary 3.15 (Saturation theorem for parabolic Kostant partition functions). 
For any positive number $N$ we have

$$
s(N \gamma, \eta)=N s(\gamma, \eta) .
$$

Conjecture 3.16 (Unimodality conjecture for parabolic Kostant partition functions).

Let $\eta$ be a composition of size $n$, and $\gamma \in \mathbb{Z}^{n}$ such that $|\gamma|=0$. Then, $K_{\Phi(\eta)}(\gamma \mid q)$ is a unimodal polynomial in the variable $q$.

\section{§3.4. Rationality and polynomiality theorems}

Theorem 3.17 (Rationality theorem for parabolic Kostant partition function, I).

Let $\eta$ be a composition and $\gamma \in Y_{\eta}$. Then

$$
\sum_{n \geq 0} K_{\Phi(\eta)}(n \gamma \mid q) t^{n}=P_{\eta \gamma}(q, t) / Q_{\eta \gamma}(q, t)
$$

where $P_{\eta \gamma}(q, t)$ and $Q_{\eta \gamma}(q, t)$ are mutually prime polynomials in $q$ and $t$ with integer coefficients, $P_{\eta \gamma}(0,0)=1$.

Moreover,

(\$) the denominator $Q_{\eta \gamma}$ has the following form:

$$
Q_{\eta \gamma}(q, t)=\prod_{j \in J}\left(1-q^{j} t\right)
$$

where $J:=J_{\eta \gamma}$ is a finite set of non-negative integer numbers, not necessarily distinct;

(20) $P_{\eta \gamma}(1, t)=(1-t)^{t(\eta, \gamma)} P_{\eta \gamma}(t), \quad P_{\eta \gamma}(1) \neq 0$, where $t(\eta, \gamma) \in \mathbb{Z}_{\geq 0}$, and $P_{\eta \gamma}(t)$ is a polynomial with non-negative integer coefficients.

( We expect that if $\gamma_{1}$ and $\gamma_{2}$ belong to the set $Y_{\eta}$, and $\gamma_{1} \geq \gamma_{2}$, i.e. $\sum_{j \leq k} \gamma_{1, j} \geq \sum_{j \leq k} \gamma_{2, j}, \forall k \geq 1$, then

$$
P_{\eta \gamma_{2}}(t)-P_{\eta \gamma_{1}}(t) \geq 0
$$

In other words, the latter difference is a polynomial with non-negative coefficients.

Corollary 3.18 (Polynomiality theorem for parabolic Kostant partition function).

Let $\eta$ be a composition and $\gamma \in Y_{\eta}$. There exists a polynomial $\mathcal{K}_{\eta \gamma}(t)$ with rational coefficients such that for any integer number $N \geq 1, \mathcal{K}_{\eta \gamma}(N)=$ $K_{\Phi(\eta)}(N \gamma)$ 
Conjecture 3.19. The polynomials $\mathcal{K}_{\eta \gamma}(t)$ have non-negative rational coefficients.

Theorem 3.20 (Rationality theorem for parabolic Kostant partition function, II).

Let $\eta$ be a composition and $\gamma_{1}, \ldots, \gamma_{k} \in Y_{\eta}$. Then the generating function

$$
\sum_{\left(N_{1}, \ldots, N_{k}\right) \in \mathbb{Z}_{\geq 0}^{k}} K_{\Phi(\eta)}\left(N_{1} \gamma_{1}+\cdots+N_{k} \gamma_{k} \mid q\right) x_{1}^{N_{1}} \ldots x_{k}^{N_{k}}
$$

is a rational function in $q$ and the variables $X_{k}=\left(x_{1}, \ldots, x_{k}\right)$ of the form $P\left(q, X_{k}\right) / Q\left(q, X_{k}\right)$, where $P:=P_{\gamma_{1}, \ldots, \gamma_{k}, \eta}\left(q, X_{k}\right)$ and $Q\left(q, X_{k}\right):=Q_{\gamma_{1}, \ldots, \gamma_{k}, \eta}$ $\left(q, X_{k}\right)$ are mutually prime polynomials in $q$ and $X_{k}$ with integer coefficients, $P(0,0)=1$.

(\$) Moreover, the denominator $Q\left(q, X_{k}\right)$ has the following structure:

$$
Q\left(q, X_{k}\right)=\prod_{\emptyset \neq W \subset\{1, \ldots, k\}} \prod_{a_{W} \in J_{W}}\left(1-q^{a_{W}} x_{W}\right),
$$

where $x_{W}:=\prod_{i \in W} x_{i}$, and for each non-empty subset $W \subset\{1, \ldots, k\}, J_{W}$ denotes a certain set, depending on $W$ and $\gamma_{1}, \ldots, \gamma_{k}$, of non-negative integers, not necessarily distinct.

(W) We expect that if $W=\{b\}, 1 \leq b \leq k$, then $J_{W}=J_{\gamma_{b}, \eta}$.

Corollary 3.21 (Piecewise polynomiality theorem for parabolic Kostant partition function).

Let $\eta$ be a composition and $\gamma_{1}, \ldots, \gamma_{k} \in Y_{\eta}$. There exists a piecewise polynomial function $\mathcal{K}\left(t_{1}, \ldots, t_{k}\right):=\mathcal{K}_{\gamma_{1}, \ldots, \gamma_{k}}\left(t_{1}, \ldots, t_{k}\right)$ with rational coefficients such that for any non-negative integer numbers $N_{1}, \ldots, N_{k}, \mathcal{K}\left(N_{1}, \ldots, N_{k}\right)=$ $K_{\Phi(\eta)}\left(N_{1} \gamma_{1}+\cdots+N_{k} \gamma_{k}\right)$.

(W) We expect that the restriction of the function $\mathcal{K}\left(t_{1}, \ldots, t_{k}\right)$ on "the dominant chamber" $\mathcal{N}_{k}:=\left\{\left(N_{1} \geq N_{2} \geq \cdots \geq N_{k}\right) \in \mathbb{Z}_{\geq 0}^{k}\right\}$ is a polynomial with non-negative rational coefficients.

Example 3.22. Take $\gamma_{1}=(2,1,0,-1,-1,-1), \gamma_{2}=(1,1,-1,-1)$ and $\eta=\left(1^{5}\right)$. Then $Q_{\gamma_{1}, \gamma_{2}}(1, x, y)=(1-x)^{7}(1-y)^{4}$, and

$$
\begin{aligned}
P_{\gamma_{1}, \gamma_{2}}(1, x, y)= & (1,26,71,26)+(1,-57,-223,-93) y \\
& +(0,33,224,115) y^{2}-(0,8,66,50) y^{3} .
\end{aligned}
$$

Therefore, in our example the function $(n, m) \rightarrow K_{\Phi\left(1^{5}\right)}(n(2,1,-1,-1,-1)+$ $m(1,1,-1,-1))$ is a polynomial one on the whole set $\left\{(n, m) \in \mathbb{Z}_{\geq 0}^{2}\right\}$. 
(1) We expect that in fact the function $\left(n_{1}, \ldots, n_{k}\right) \rightarrow K_{\Phi(\eta)}\left(n_{1} \gamma_{1}+\right.$ $\left.\cdots+n_{k} \gamma_{k}\right)$ is a polynomial one on the whole set $\left\{\left(n_{1}, \ldots, n_{k}\right) \in \mathbb{Z}_{\geq 0}^{k}\right\}$, cf mixed lattice point enumerator theorem by P. McMullen [54].

\section{§3.5. Parabolic Kostant partition function $K_{\Phi(\eta)}(\gamma)$ as function of $\gamma$}

In this Section we state a few theorems, problems and one conjecture about behavior of the parabolic Kostant partition function $K_{\Phi(\eta)}(\gamma)$, considered as a function of $\gamma$, on the set $Y_{\eta}$.

Theorem 3.23 (Polynomial expression for the restriction of the parabolic Kostant partition function $K_{\Phi(\eta)}(\gamma)$ on the dominant chamber $\left.Y_{\eta}^{+}\right)$.

Let $\eta=\left(\eta_{1}, \ldots, \eta_{p}\right), p \geq 3, \eta_{p} \neq 0$, be a composition, consider vector $l=\left(l_{1}, l_{2}, \ldots, l_{r_{p-2}}\right)$, where $l_{i}=\sum_{j=k+1}^{p-1} \eta_{j}$ if $r_{k-1}<i \leq r_{k}, 1 \leq k \leq p-2$. Let $\widehat{\eta}=\left(\eta_{1}, \ldots, \eta_{p-2}\right)$. If $\gamma \in Y_{\eta}^{+}$, then

$$
K_{\Phi(\eta)}(\gamma)=\sum_{\beta} K_{\Phi(\widehat{\eta})}\left(\beta_{1}-l_{1}, \ldots, \beta_{r_{p-2}}-l_{r_{p-2}}\right) \prod_{j=1}^{r_{p-2}}\left(\begin{array}{c}
\gamma_{j}+l_{j} \\
\beta_{j}
\end{array}\right)
$$

where the sum runs over $\beta \in \mathbb{Z}_{\geq 0}^{r_{p-2}}$ such that $|\beta|=|l|=\sum_{1 \leq i<j \leq p-1} \eta_{i} \eta_{j}$.

Corollary 3.24. Being restricted on the dominant chamber $Y_{\eta}^{+}$, the function $F_{\eta}(\gamma):=K_{\Phi(\eta)}(\gamma)$ is a polynomial in $\gamma_{1}, \ldots, \gamma_{r_{p-2}}$ of degree $|l|=$ $\sum_{1 \leq i<j \leq p} \eta_{i} \eta_{j}-\eta_{p}\left(n-\eta_{p}\right)$ with rational coefficients.

Theorem 3.25 (Piecewise polynomiality theorem for function $\left.\gamma \longrightarrow K_{\Phi(\eta)}(\gamma)\right)$.

On the set $Y_{\eta}$ the function $\gamma \longrightarrow F_{\eta}(\gamma):=K_{\Phi(\eta)}(\gamma)$ is a continuous piecewise polynomial function of degree $\sum_{1 \leq i<j \leq p} \eta_{i} \eta_{j}-n+1$.

We see that if $\eta_{p}>1$, then the dominant chamber $Y_{\eta}^{+}$is strictly contained in some maximal polynomiality domains of the function $F_{\eta}$.

Problem 3.26. $\quad$ Count the number and describe a structure of the polynomiality domains of the function $F_{\eta}$.

Conjecture 3.27. Restriction of the function $|l| ! F_{\eta}$ on the dominant chamber $Y_{\eta}^{+}$, denoted by $F_{\eta}^{+}$, is a polynomial in $\gamma_{1}, \ldots, \gamma_{r_{p-2}}$ with non-negative integer coefficients. 
Problem 3.28. $\quad$ Find a combinatorial interpretation of the coefficients of the polynomial $F_{\eta}^{+}$.

\section{§3.6. Reconstruction theorem}

The leading term $|l| ! G_{\eta}(\gamma)$ of the polynomial $F_{\eta}^{+}(\gamma)$, i.e. the degree $|l|$ homogeneous part of $F_{\eta}^{+}(\gamma)$, admits the following description.

Definition 3.29. For any composition $\eta=\left(\eta_{1}, \ldots, \eta_{p}\right)$, such that $\eta_{p}>$ 0 and $p \geq 3$, define the operator

$$
\mathcal{D}_{\eta}=\prod_{1 \leq i \leq \eta_{1}<j \leq r_{p-2}}\left(\partial / \partial \gamma_{i}-\partial / \partial \gamma_{j}\right)
$$

acting on the quotient ring of the ring of polynomials $\mathbb{Q}\left[\gamma_{1}, \ldots, \gamma_{n}\right]$ by the ideal generated by the sum $\gamma_{1}+\cdots+\gamma_{n}$.

Let $\gamma=\left(\gamma_{1}, \ldots, \gamma_{n}\right) \in \mathbb{Z}^{n},|\gamma|=0$.

Theorem 3.30 (Characterization of polynomials $G_{\eta}(\gamma)$ ).

The polynomials $G_{\eta}(\gamma)$ are uniquely determined by the following properties

(i) $G_{\eta}(\gamma)$ is a homogeneous polynomial of degree $|l|=\sum_{1 \leq i<j \leq p-1} \eta_{i} \eta_{j}$,

(ii) $\mathcal{D}_{\eta} G_{\eta}(\gamma)=\prod_{j=1}^{\eta_{1}}\left(\gamma_{j}^{\eta_{p-1}} / \eta_{p-1} !\right) G_{\left(\eta_{2}, \ldots, \eta_{p}\right)}\left(\gamma_{\eta_{1}+1}, \ldots, \gamma_{n}\right)$,

(iii) $G_{\eta_{1}, \eta_{2}}(\gamma)=1$.

\section{Theorem 3.31 (Reconstruction Theorem).}

Let $G_{\eta}(\gamma)=\sum_{\beta} b_{\eta}(\beta) \prod_{j=1}^{r_{p-2}} \gamma_{j}^{\beta_{j}} / \beta_{j}$ !, summed over $\beta \in \mathbb{Z}_{\geq 0}^{r_{p-2}}$ such that $|\beta|=|l|$. Then

$$
F_{\eta}(\gamma)=\sum_{\beta} b_{\eta}(\beta) \prod_{j=1}^{r_{p-2}}\left(\begin{array}{c}
\gamma_{j}+l_{j} \\
\beta_{j}
\end{array}\right) .
$$

Corollary 3.32. $\quad$ Let $l$ be the vector defined in Theorem 3.23, then

$$
b_{\eta}(\beta)=K_{\Phi(\eta)}(\beta-l)
$$

In particular, $G_{\eta}(\gamma)$ is a polynomial with non-negative rational coefficients.

Finally, we state a result which is a refinement of Proposition 3.7, and gives partly a $q$-analog of the recurrence relation (3.32). 
Theorem 3.33 (A $q$-analog of Theorem 3.23).

Let $\eta=\left(\eta_{1}, \ldots, \eta_{p}\right), p \geq 3, \eta_{p} \neq 0$, be a composition. Define $\widehat{\gamma}=$ $(\gamma_{1}, \ldots, \gamma_{r_{p-3}} \underbrace{0, \ldots, 0}_{\eta_{p-2}})$ and $\hat{\eta}=\left(\eta_{1}, \ldots, \eta_{r_{p-2}}\right)$. If $\gamma \in Y_{\eta}^{+}$, then

$K_{\Phi(\eta)}(\gamma \mid q)=q^{-\gamma_{n}} \sum_{\beta} K_{\Phi(\widehat{\eta})}(\widehat{\gamma}-\beta \mid q) \prod_{j=1}^{r_{p-3}} B_{q}\left(\beta_{j}+\eta_{p-1} ; \eta_{p-1}\right)$
$\prod_{j=r_{p-3}+1}^{r_{p-2}} B_{q}\left(\gamma_{j}+\beta_{j}+\eta_{p-1} ; \eta_{p-1}\right)$,

where the sum runs over vectors $\beta \in \mathbb{Z}_{\geq 0}^{r_{p-2}}$ such that $|\beta|=\sum_{j=1}^{r_{p-3}} \gamma_{j}$, and polynomials $B_{q}(n ; k)$ are defined in Proposition 3.8, formula (3.30).

Remark 3.34. The "classical" case $\eta=\left(1^{n}\right)$ and $q=1$, which corresponds to the Kostant partition function $K_{n}(\gamma)$, has been studied by F. Berezin and I.M Gelfand [5], B. Kostant [45], B.V. Lidskii [50], [51], D. Peterson, A.N. K. [34], [35], A. Postnikov and R. Stanley [68], W. Baldoni-Silva and M. Vergne [2], S. Billey, V. Guillemin and E. Rassart [8], J. De Loera and B. Sturmfels $[13], \ldots$. In particular, if $\eta=\left(1^{n}\right)$ and $q=1$, Theorem 3.19 has been proved by B.V. Lidskii [50] in 1984, and by D. Peterson (unpublished). The case of arbitrary $\eta$ and $q$ has been studied by the author (unpublished, but see [35]). The case of generalized Kostant partition functions and $q=1$ has been studied by A. Postnikov and R. Stanley (unpublished, but see [68]).

\section{§4. Parabolic Kostka Polynomials: Definition and Basic Properties}

Definition 4.1 ([35], [41]). Let $\lambda$ be a partition and $\mu$ and $\eta$ be compositions such that $|\lambda|=|\mu|,|\eta|=n$ and $l l(\mu) \leq n$. Define the parabolic Kostka polynomial $K_{\lambda \mu \eta}(q)$ as follows:

$$
K_{\lambda \mu \eta}(q):=\sum_{w \in \Sigma_{n}}(-1)^{l(w)} K_{\Phi(\eta)}(w(\lambda+\delta)-\mu-\delta \mid q),
$$

where $\delta:=\delta_{n}=(n-1, n-2, \ldots, 1,0)$.

If a composition $\mu$ is compatible with $\eta$ and corresponds to the sequence of partitions (possibly with zeros at the end) $\boldsymbol{\mu}=\left(\mu^{(1)}, \mu^{(2)}, \ldots, \mu^{(r)}\right)$, we will denote the parabolic Kostka polynomial $K_{\lambda \mu \eta}(q)$ by $K_{\lambda, \boldsymbol{\mu}}(q)$ or $K_{\lambda,\left(\mu^{(1)}, \mu^{(2)}, \ldots, \mu^{(r)}\right)}(q)$. If a sequence of partitions $\boldsymbol{\mu}=\left(\mu^{(1)}, \ldots, \mu^{(r)}\right)$ consists of only rectangular shape partitions $\mu^{(a)}=\left(\mu_{a}^{\eta_{a}}\right):=R_{a}, \quad 1 \leq a \leq r$, we will write $R=\left(R_{1}, R_{2}, \ldots, R_{r}\right)$ instead of $\boldsymbol{\mu}$, and $K_{\lambda, R}(q)$ instead of $K_{\lambda, \boldsymbol{\mu}}(q)$.

Let us elucidate Definition 4.1 by a simple, but interesting example.

Example 4.2. Take $\lambda=(6,2,2,2), \mu=\left(2^{6}\right)$ and $\eta=\left(2^{3}\right)$. There are 4 contributions to the $\operatorname{RHS}(4,33)$, namely,

$$
K_{\lambda \mu \eta}(q)=K_{\Phi(\eta)}\left(\gamma_{1} \mid q\right)-K_{\Phi(\eta)}\left(\gamma_{2} \mid q\right)-K_{\Phi(\eta)}\left(\gamma_{3} \mid q\right)+K_{\Phi(\eta)}\left(\gamma_{4} \mid q\right)
$$


where $\gamma_{1}=\lambda-\mu=(4,0,0,0,-2,-2), \gamma_{2}=(4,0,0,0,-3,-1), \gamma_{3}=(4,0,-1$, $1,-2,-2)$ and $\gamma_{4}=(4,0,-1,1,-3,-1)$. It is not difficult to see that $K_{\Phi(\eta)}$ $\left(\gamma_{1} \mid q\right)=q^{4}(1,4,10,12,9), K_{\Phi(\eta)}\left(\gamma_{2} \mid q\right)=q^{4}(1,4,7,10,8), K_{\Phi(\eta)}\left(\gamma_{3} \mid q\right)=$ $q^{5}(2,7,10,7)$ and $K_{\Phi(\eta)}\left(\gamma_{4} \mid q\right)=q^{5}(2,5,8,6)$. Hence, $K_{\lambda \mu \eta}(q)=q^{6}$, and $\operatorname{deg} K_{\lambda \mu \eta}(q)=6<\operatorname{deg} K_{\Phi(\eta)}(\lambda-\mu \mid q)=8$.

Remark 4.3. Using in Definition 4.1 the $q$-analog $K_{\Sigma}(\gamma \mid q)$ of the generalized Kostant partition function, see Section 3.1, Remark 3.4, one can define the "generalized" Kostka polynomials $K_{\lambda \mu \Sigma}(\gamma \mid q)$. They form an interesting family of polynomials to study.

Theorem 4.4 ([64]). Let $\lambda$ be a partition, and $\mu$ be a composition compatible with $\eta$. Then

$$
K_{\lambda, \boldsymbol{\mu}}(1):=K_{\lambda,\left(\mu^{(1)}, \mu^{(2)}, \ldots, \mu^{(r)}\right)}(1)=\operatorname{Mult}\left[V_{\lambda}: \otimes_{i=1}^{r} V_{\mu^{(i)}}\right]
$$

i.e. $K_{\lambda,\left(\mu^{(1)}, \mu^{(2)}, \ldots, \mu^{(r)}\right)}(1)$ is equal to the multiplicity of the irreducible highest weight $\lambda \mathfrak{g l}(n)$-module $V_{\lambda}$ in the tensor product of irreducible highest weight $\mu^{(i)}$ representations $V_{\mu^{(i)}}, 1 \leq i \leq r$, of the Lie algebra $\mathfrak{g l}(n)$.

In the case when all partitions $\mu^{(i)}$ have rectangular shapes, Theorem 4.4 has been proved in [28].

Remark 4.5. We expect that $K_{\lambda \mu \eta}(1) \geq 0$ for any partition $\lambda$ and compositions $\mu$ and $\eta$. It seems a challenge problem to find a combinatorial and/or representation-theoretic interpretations of the numbers $K_{\lambda \mu \eta}(1)$ and $K_{\lambda \mu \eta}(-1)$ for general $\lambda, \mu$ and $\eta$. In particular,

(\$) When does the number $K_{\lambda \mu \eta}(1)$ equal to 1 ?

Examples 4.6. In these examples we will use notation $P_{\lambda \mu \eta}(q, t), Q_{\lambda \mu \eta}$ $(q, t)$ and $J_{\lambda \mu \eta}(q)$, which will be explained in Theorem 4.14 .

(i) Take $\lambda=(3,2,1), \mu=(2,2,2)$ and $\eta=\left(1^{3}\right)$. Then $K_{\lambda \mu \eta}(q)=K_{\lambda \mu}(q)=$ $q+q^{2}$, and

$$
\sum_{n \geq 0} K_{n \lambda, n \mu, \eta}(q) t^{n}=(1-q t)^{-1}\left(1-q^{2} t\right)^{-1}
$$

(ii) Take the same $\lambda$, but $\mu=(0,2,2,2)$ and $\eta=\left(1^{4}\right)$. Then

$$
K_{\lambda \mu \eta}(q)=q^{3}(-1,-1,0,1,2,1), K_{2 \lambda, 2 \mu, \eta}(q)=q^{5}(1,0,-2,-4,-4,-1,0,3,
$$
$3,4,2,1)$. 
Moreover,

$$
\begin{aligned}
& P_{\lambda \mu \eta}(q, t)=1-q^{2}(1,3,2,1) t+\cdots+q^{33}(-1,1,1,0,-1,-1) t^{7}, \\
& Q_{\lambda \mu \eta}(q, t)=\left(1-q^{3} t\right)\left(1-q^{7} t\right) \prod_{j=2}^{8}\left(1-q^{j} t\right), \quad J_{\lambda \mu \eta}(q)=q^{2}(1,2,1,1,1,2,1),
\end{aligned}
$$

see Theorem 4.14, (\$), for the definition of polynomials $J_{\lambda \mu \eta}(q)$.

(iii) Take again $\lambda=(3,2,1)$, but $\mu=(0,2,0,2,2)$ and $\eta=(1,2,1,1)$. Then $K_{\lambda \mu \eta}(q)=q^{3}(1,0,-4-3,2,4,2), K_{2 \lambda, 2 \mu, \eta}(q)=q^{7}(3,5,6,-3,-13,-17$, $-11,3,9,12,6,3)$. Moreover,

$$
\begin{aligned}
& P_{\lambda \mu \eta}(q, t)=1-q^{3}(1,2,6,5,0,-2) t+\cdots+q^{70}(1,-1,-2,1,2) t^{12}, \\
& Q_{\lambda \mu \eta}(q, t)=\prod_{j=3}^{9}\left(1-q^{j} t\right)^{2}, \quad J_{\lambda \mu \eta}(q)=q^{3}(2,2,2,2,2,2,2) .
\end{aligned}
$$

(iv) Take the same $\lambda=(3,2,1)$, but $\mu=(0,2,0,2,0,2)$ and $\eta=(1,2,2,1)$. Then $K_{\lambda \mu \eta}(q)=q^{4}(1,2,-8,-6,8,5), K_{2 \lambda, 2 \mu, \eta}(q)=-q^{7}-2 q^{8}+\cdots+22 q^{17}+12 q^{18}$. Moreover, $P_{\lambda \mu \eta}(q, t)=1-q^{3}(2,3,2,11,10,-4,-2) t+\cdots+q^{132}(1,-1,-2,1,2)$ $t^{22}$, and $J_{\lambda \mu \eta}(q)=q^{3}(2,4,4,3,4,4,3)$. In other words,

$Q_{\lambda \mu \eta}(q, t)=\left(1-q^{3} t\right)^{2}\left(1-q^{4} t\right)^{4}\left(1-q^{5} t\right)^{4}\left(1-q^{6} t\right)^{3}\left(1-q^{7} t\right)^{4}\left(1-q^{8} t\right)^{4}\left(1-q^{9} t\right)^{3}$.

(\$) We would like to remark that the reasons for the equality below are elusive.

$$
\left.q^{62} P_{(3,2,1),(0,2,0,2,2),(1,2,1,1)}(q, t)\right|_{t^{12}}=\left.P_{(3,2,1),(0,2,0,2,0,2),(1,2,2,1)}(q, t)\right|_{t^{22}} .
$$

(A) These examples show that for general $\lambda, \mu$ and $\eta$, the polynomials $K_{\lambda \mu \eta}(q)$ may have negative coefficients, the numbers $a(\lambda, \mu \| \eta)$ may be negative and may not be a homogeneous function in $n$, and those $b(\lambda, \mu \| \eta)$ may not satisfy the (generalized) Fulton conjecture.

Our nearest goal is to describe several cases when the polynomials $K_{\lambda \mu \eta}(q)$ have only non-negative coefficients. However, we want to point out that there are many other cases when the all coefficients of a parabolic Kostka polynomial are non-negative.

Example 4.7. Take $\lambda=(6,3,2,1), \mu=(2,1,2,1,2,1,2,1)$ and $\eta=$ $\left(2^{4}\right)$. Then $K_{\lambda \mu \eta}(q)=q^{11}(4,18,24,14,4)$. It is interesting to compare the 
polynomial $K_{\lambda \mu \eta}(q)$ with the $q$-analog of the $L R$-numbers $c_{\mu^{(1)}, \ldots, \mu^{(r)}}^{\lambda}(q)$ introduced by C. Carre, A. Lascoux, B. Leclerc and J.-Y. Thibon, see e.g. [47]. Namely, one can show that $c_{(2,1),(2,1),(2,1),(2,1)}^{(6,3,2,1)}(q)=q^{8}(2,7,12,15,14,9,4,1)$.

Proposition 4.8. Let $\lambda$ be a partition and $\boldsymbol{\mu}=\left(\mu^{(1)}, \ldots, \mu^{(r)}\right)$ be a sequence of partitions.

If inequalities $l l\left(\mu^{(i)}\right) \geq l(\lambda)$ holds for all $i$, then

$$
K_{\lambda, \boldsymbol{\mu}}(q) \doteq \operatorname{Mult}\left[V_{\lambda}: \otimes_{i=1}^{r} V_{\mu^{(i)}}\right] .
$$

Proposition 4.9. Let $\lambda$ be a partition and $\boldsymbol{\mu}=\left(\mu^{(1)}, \mu^{(2)}\right)$ be a dominant sequence of partitions. Then

$$
K_{\lambda, \boldsymbol{\mu}}(q) \doteq c_{\mu^{(1)}, \mu^{(2)}}^{(\lambda)} .
$$

See Introduction, Section 1.1, for the explanation of the meaning of the symbol "․ㅡ".

Positivity Theorem ([29], [42]). Let $\lambda$ be a partition, and $\boldsymbol{\mu}=\left(\mu^{(1)}\right.$, $R_{2}, \ldots, R_{r}$ ) be a sequence of (proper) partitions such that

(a) $\left(R_{2}, \ldots, R_{r}\right)$ is a dominant sequence of rectangular shape partitions,

(b) either $l l\left(\mu^{(1)}\right) \geq l(\lambda)$,

or $\lambda \supset \mu^{(1)}$ and the complement $\lambda \backslash \mu^{(1)}$ is a disjoint union of partitions $\lambda^{(1)}, \lambda^{(2)}, \ldots, \lambda^{(p)}$.

Then the parabolic Kostka polynomial $K_{\lambda,\left(\mu^{(1)}, R_{2}, \ldots, R_{r}\right)}(q)$ has non-negative integer coefficients.

Conjecture 4.10 (Positivity conjecture for parabolic Kostka polynomials, cf [32], [41]).

Let $\lambda$ be a partition and $\boldsymbol{\mu}=\left(\mu^{(1)}, \mu^{(2)}, \ldots, \mu^{(r)}\right)$ be a sequence of (proper) partitions such that $\left(\mu^{(2)}, \ldots, \mu^{(r)}\right)$ is a dominant sequence of partitions. Assume that

either $\lambda \supset \mu^{(1)}$ and the complement $\lambda \backslash \mu^{(1)}$ is a disjoint union of partitions $\lambda^{(1)}, \lambda^{(2)}, \ldots, \lambda^{(p)}$, or $l l\left(\mu^{(1)}\right) \geq l(\lambda)$.

Then

$$
K_{\lambda, \boldsymbol{\mu}}(q) \in \mathbb{N}[q]
$$

(\$) In particular, we expect [32], [41] that if $\lambda$ and $\mu$ are partitions and $\eta$ is a composition, then

$$
K_{\lambda \mu \eta}(q) \in \mathbb{N}[q]
$$


Remark 4.11. According to (4.34) and Conjecture 4.10, if $\mu$ is a (proper) partition, then the parabolic Kostka polynomials $K_{\lambda \mu \eta}(q)$ may be considered as a $q$-analog of the tensor product multiplicities. Another $q$-analog of the tensor product multiplicities has been introduced by C. Carre and B. Leclerc [11], and A. Lascoux, B. Leclerc and J.-Y. Thibon [47]. Formulas (4.35) and (4.36) show that in general these two $q$-analogs are different. However, it was conjectured in [32], Conjecture 6.5 and in [41], Conjecture 5, that, in fact, these two $q$-analogs coincide in the case when a partition $\mu$ and a composition $\eta$ correspond to a dominant sequence of rectangular shape partitions.

Duality Theorem $([33,41])$. Let $\lambda$ be a partition, and $R$ be a dominant sequence of rectangular shape partitions, $R=\left(\left(\mu_{a}^{\eta_{a}}\right)\right)_{a=1}^{r}$. Denote by $R^{\prime}$ a dominant rearrangement of the sequence of rectangular shape partitions $\left(\left(\eta_{a}^{\mu_{a}}\right)\right)_{a=1}^{r}$ obtained by transposing each of the rectangular in $R$. Then

$$
K_{\lambda^{\prime} R^{\prime}}(q)=q^{n(R)} K_{\lambda R}\left(q^{-1}\right)
$$

where $n(R)=\sum_{1 \leq a<b \leq p} \min \left(\mu_{a}, \mu_{b}\right) \min \left(\eta_{a}, \eta_{b}\right)$.

Note that the left hand side of (4.37) is computed in $\mathfrak{g l}(m)$, where $m=$ $\sum \mu_{a}$ is the total number of columns in the rectangles of $R$, whereas the right hand side of (4.37) is computed in $\mathfrak{g} l(n)$, where $n=\sum \eta_{a}$ is the total number of rows in the rectangles of $R$.

Corollary 4.12. We have

(i) $a(\lambda, R)=n(R)-c\left(\lambda^{\prime}, R^{\prime}\right)$,

(ii) $b(\lambda, R)=d\left(\lambda^{\prime}, R^{\prime}\right)$

Conjecture 4.13. Let $\lambda$ and $\mu$ be partitions, and $\eta_{1}$ and $\eta_{2}$ be compositions such that $\eta_{2}$ is a subdivision of $\eta_{1}$. Then

$$
K_{\lambda \mu \eta_{1}}(q) \leq K_{\lambda \mu \eta_{2}}(q)
$$

We remark that Conjecture 4.13 is false if one assumes only that $\eta_{1} \geq \eta_{2}$ with respect to the dominance partial ordering on the set of compositions, see Example 3.6.

Theorem 4.14 (Rationality theorem for parabolic Kostka polynomials, I).

The formal power series

$$
\sum_{n \geq 0} K_{n \lambda, n \mu, \eta}(q) t^{n}
$$


is a rational function in $q$ and $t$ of the form

$$
P_{\lambda \mu \eta}(q, t) / Q_{\lambda \mu \eta}(q, t)
$$

where $P_{\lambda \mu \eta}(q, t)$ and $Q_{\lambda \mu \eta}(q, t)$ are mutually prime polynomials in $q$ and $t$ with integer coefficients and $P_{\lambda \mu \eta}(0,0)=1$.

Moreover,

(\$) the denominator $Q_{\lambda \mu \eta}$ has the following form:

$$
Q_{\lambda \mu \eta}(q, t)=\prod_{j \in J}\left(1-q^{j} t\right)
$$

where $J:=J_{\lambda \mu \eta}$ is a finite set of non-negative integer numbers, not necessarily distinct;

(30) $P_{\lambda \mu \eta}(1, t)=(1-t)^{t(\lambda, \mu, \eta)} P_{\lambda \mu \eta}(t)$, where $t(\lambda, \mu, \eta) \in \mathbb{Z}_{\geq 0}, \quad P_{\lambda \mu \eta}(1) \neq$ 0 , and $P_{\lambda \mu \eta}(t)$ is a polynomial with non-negative integer coefficients.

(\$) It is convenient to depict the set $J_{\lambda \mu \eta}$ in the polynomial $J_{\lambda \mu \eta}(q)=$ $\sum_{j \in J_{\lambda \mu \eta}} q^{j}$.

( $\mathbf{\Psi})$ We expect that if $\mu_{1}$ and $\mu_{2}$ are partitions such that $\mu_{1} \geq \mu_{2}$ with respect to the dominance partial ordering, see e.g. Section 2.1, then

$$
P_{\lambda, \mu_{2}, \eta}(t)-P_{\lambda, \mu_{1}, \eta}(t) \geq 0
$$

i.e. the latter difference is a polynomial with non-negative coefficients.

Corollary 4.15 (Polynomiality theorem for parabolic Kostka numbers).

Let $\lambda$ be a partition, and $\mu$ and $\eta$ be compositions such that $\lambda-\mu \in Y_{\eta}$. There exists a polynomial $\mathcal{K}_{\eta \mu \eta}(t)$ with rational coefficients such that

(\$) for any integer number $N \geq 1, \mathcal{K}_{\eta \mu \eta}(N)=K_{N \lambda, N \mu, \eta}(1)$.

Conjecture 4.16. If $\mu$ is a partition and $\eta$ is a composition, then the polynomial $\mathcal{K}_{\eta \mu \eta}(t)$ has non-negative rational coefficients.

Theorem 4.14 is a corollary of the corresponding theorem for parabolic Kostant's partition function (Theorem 3.17) . In Section 6, Rationality Conjecture, we state a few conjectures about the structure of the numerator $P_{\lambda \mu \eta}(q, t)$. 
Theorem 4.17 (Rationality theorem for parabolic Kostka polynomials, II).

Let $\boldsymbol{\lambda}=\left(\lambda^{(1)}, \ldots, \lambda^{(k)}\right)$ be a sequence of partitions, $\boldsymbol{\mu}=\left(\mu^{(1)}, \ldots, \mu^{(k)}\right)$ be a sequence of compositions and $\eta$ be a composition such that $\left|\lambda^{(j)}\right|=\left|\mu^{(j)}\right|$ and $l l\left(\mu^{(j)}\right) \leq|\eta|$ for all $1 \leq j \leq k$. Then the generating function

$$
\sum_{\left(n_{1}, \ldots, n_{k}\right) \in \mathbb{Z}_{\geq 0}^{k}} K_{n_{1} \lambda^{(1)}+\cdots+n_{k} \lambda^{(k)}, n_{1} \mu^{(1)}+\cdots+n_{k} \mu^{(k)}, \eta}(q) x_{1}^{n_{1}} \ldots x_{k}^{n_{k}}
$$

is a rational function in $q$ and the variables $X_{k}:=\left(x_{1}, \ldots, x_{k}\right)$ of the form $P\left(q, X_{k}\right) / Q\left(q, X_{k}\right)$, where $P\left(q, X_{k}\right):=P_{\boldsymbol{\lambda}, \boldsymbol{\mu}, \eta}\left(q, X_{k}\right)$ and $Q\left(q, X_{k}\right):=Q_{\boldsymbol{\lambda}, \boldsymbol{\mu}, \eta}$ $\left(q, X_{k}\right)$ are mutually prime polynomials in $q$ and $X_{k}$ with integer coefficients, $P(0,0)=1$.

(\$) Moreover, the denominator $Q\left(q, X_{k}\right)$ has the following structure:

$$
Q\left(q, X_{k}\right)=\prod_{\emptyset \neq W \subset\{1, \ldots, k\}} \prod_{a_{W} \in J_{W}}\left(1-q^{a_{W}} x_{W}\right),
$$

where $x_{W}:=\prod_{i \in W} x_{i}$, and for each non-empty subset $W \subset\{1, \ldots, k\}, J_{W}$ denotes a certain set, depending on $W$ and $\boldsymbol{\lambda}, \boldsymbol{\mu}, \eta$, of non-negative integer numbers, not necessarily distinct.

( We expect that in general, all the sets $J_{W}, \emptyset \neq W \subset\{1, \ldots, k\}$, are non trivial, i.e. each contain at least one positive element.

Corollary 4.18 (Piecewise polynomiality theorem for parabolic Kostka numbers).

Let $\boldsymbol{\lambda}=\left(\lambda^{(1)}, \ldots, \lambda^{(k)}\right)$ be a sequence of partitions, $\boldsymbol{\mu}=\left(\mu^{(1)}, \ldots, \mu^{(k)}\right)$ be a sequence of compositions and $\eta$ be a compositions such that $\left|\lambda^{(j)}\right|=\left|\mu^{(j)}\right|$ and $l l\left(\mu^{(j)}\right) \leq|\eta|$ for all $1 \leq j \leq k$. There exists a piecewise polynomial function $\mathcal{K}\left(t_{1}, \ldots, t_{k}\right):=\mathcal{K}_{\boldsymbol{\lambda}, \boldsymbol{\mu}, \eta}\left(t_{1}, \ldots, \gamma_{k}\right)$ with rational coefficients such that for any non-negative integer numbers $N_{1}, \ldots, N_{k}$,

$$
\mathcal{K}\left(N_{1}, \ldots, N_{k}\right)=K_{N_{1} \lambda^{(1)}+\cdots+N_{k} \lambda^{(k)}, N_{1} \mu^{(1)}+\cdots+N_{k} \mu^{(k)}, \eta}(1) .
$$

( We expect that if all $\mu^{(1)}, \ldots, \mu^{(k)}$ are partitions, then the restriction of the function $\mathcal{K}_{\boldsymbol{\lambda}, \boldsymbol{\mu}, \eta}\left(t_{1}, \ldots, t_{k}\right)$ on "the dominant chamber" $\mathcal{N}_{k}:=\left\{\left(N_{1} \geq\right.\right.$ $\left.\left.N_{2} \geq \cdots \geq N_{k}\right) \in \mathbb{Z}_{\geq 0}^{k}\right\}$ is a polynomial with non-negative rational coefficients. 
Corollary 4.19 (Piecewise polynomiality theorem for $L R$ numbers).

Let $\boldsymbol{\lambda}=\left(\lambda^{(1)}, \ldots, \lambda^{(k)}\right), \boldsymbol{\mu}=\left(\mu^{(1)}, \ldots, \mu^{(k)}\right)$ and $\boldsymbol{\nu}=\left(\nu^{(1)}, \ldots, \nu^{(k)}\right)$ be three sequences of partitions. There exists a piecewise polynomial function $L R \boldsymbol{\boldsymbol { \lambda }}_{\boldsymbol{\mu}}^{\boldsymbol{\mu}}\left(t_{1}, \ldots, t_{k}\right)$ such that for any non-negative integers $N_{1}, \ldots, N_{k}$,

$$
L R_{\boldsymbol{\lambda}, \boldsymbol{\mu}}^{\boldsymbol{\nu}}\left(N_{1}, \ldots, N_{k}\right)=c_{N_{1} \lambda^{(1)}+\cdots+N_{k} \lambda^{(k)}, N_{1} \mu^{(1)}+\cdots+N_{k} \mu^{(k)}}^{N_{1} \nu^{(1)}+\cdots+N_{k} \nu^{(k)}}
$$

(W) We expect that the restriction of the function $L R \boldsymbol{\boldsymbol { \lambda }}_{\boldsymbol{\lambda}, \boldsymbol{\mu}}^{\boldsymbol{\nu}}\left(t_{1}, \ldots, t_{k}\right)$ on "the dominant chamber" $\mathcal{N}_{k}:=\left\{\left(N_{1} \geq N_{2} \geq \cdots \geq N_{k}\right) \in \mathbb{Z}_{\geq 0}^{k}\right\}$ is a polynomial with non-negative rational coefficients.

Problem 4.20. Describe the polynomiality domains of the function

$$
\left(N_{1}, \ldots, N_{k}\right) \rightarrow c_{N_{1} \lambda^{(1)}+\cdots+N_{k} \lambda^{(k)}, N_{1} \mu^{(1)}+\cdots+N_{k} \mu^{(k)}}^{N_{1} \nu^{(1)}+\cdots+N_{k} \nu^{(k)}}
$$

Examples 4.21. (i) Take $\lambda=(5,3,3,2), \mu=(3,3,3,2,1,1)$ and $\eta=\left(1^{6}\right)$.

One can check that

$K_{\lambda \mu \eta}(q)=K_{\lambda \mu}(q)=q^{3}(3,5,8,6,5,2,1), P_{\lambda \mu \eta}(q, t)=1+q^{4}(3,5,4,3,1)$ $t-q^{7}(1,3,2,1,0,1,3,3,2,1) t^{2}-q^{12}(2,9,14,18,18,20,17,14,8,4,1,1) t^{3}+q^{16}$ $(3,6,10,17,28,35,39,36,30,24,19,11,5,1) t^{4}-q^{21}(-1,0,4,3,6,6,13,16,16,10$, $5,1,1) t^{5}-q^{26}(1,2,7,10,16,19,22,23,23,20,17,10,6,4,1) t^{6}+q^{33}(1,4,8,14,17$, $20,23,23,19,16,6,1) t^{7}-q^{40}(-1,-1,1,4,6,7,3) t^{8}-q^{48}\left(1+q+q^{2}\right)^{2} t^{9}$,

$J_{\lambda \mu \eta}(q)=q^{3}(3,2,3,2,2,1,1)$. In other words,

$Q_{\lambda \mu \eta}(q, t)=\left(1-q^{3} t\right)^{3}\left(1-q^{4} t\right)^{2}\left(1-q^{5} t\right)^{3}\left(1-q^{6} t\right)^{2}\left(1-q^{7} t\right)^{2}\left(1-q^{8} t\right)\left(1-q^{9} t\right)$.

Therefore, the dimension of the Gelfand-Tsetlin polytope $G T(\lambda, \mu)$ is equal to 9 , and

$$
\begin{aligned}
\sum_{n \geq 0} K_{n \lambda, n \mu}(1) t^{n} & =\left(1+21 t+78 t^{2}+64 t^{3}+9 t^{4}\right) /(1-t)^{10}, \\
\sum_{n \geq 0} K_{n \lambda, n \mu}(-1) t^{n} & =\left(1-3 t+6 t^{2}-4 t^{3}+t^{4}\right) /\left(1-t^{2}\right)^{4}(1+t) .
\end{aligned}
$$

(ii) Take $\lambda=(3,2,1)$ and $\mu=\eta=\left(1^{6}\right)$. Then $K_{\lambda \mu}(q)=q^{4}(1,2,2,3,3$, $2,2,1), P_{\lambda \mu \eta}(q, t)=1+q^{6}(1,2,2,1,1) t+q^{12}(1,2,5,4,6,4,3,1,1) t^{2}+q^{20}(1,1,1$, $0,1,-2,-1,-2,-1,-1) t^{3}-q^{29}(2,2,4,4,4,3,3,1) t^{4}-q^{37}(1,1,2,1,2,1,1) t^{5}$,

$$
J_{\lambda \mu \eta}(q)=q^{4}(1,2,1,1,1,1,1,1) .
$$


Therefore, the dimension of the Gelfand-Tsetlin polytope $G T(\lambda, \mu)$ is equal to 7 , and

$$
\begin{aligned}
\sum_{n \geq 0} K_{n \lambda, n \mu}(1) t^{n} & =\left(1+8 t+35 t^{2}+32 t^{3}+9 t^{4}\right) /(1-t)^{8} \\
\sum_{n \geq 0} K_{n \lambda, n \mu}(-1) t^{n} & =\left(1+5 t^{2}+3 t^{4}\right) /\left(1-t^{2}\right)^{4}
\end{aligned}
$$

Remark 4.22. We see that in both examples $J_{\lambda \mu \eta}(q) \leq K_{\lambda \mu \eta}(q)$, and the initial and the leading terms of the polynomials $J_{\lambda \mu \eta}(q)$ and $K_{\lambda \mu \eta}(q)$ are the same. These observations may be not true if $\mu$ is an arbitrary composition, e.g. if $\lambda=(3,2,1), \mu=(0,2,0,2,0,2)$ and $\eta=(1,2,2,1)$, then

$K_{\lambda \mu \eta}(q)=q^{4}(1,2,-8,-6,8,5)$, but $J_{\lambda \mu \eta}(q)=q^{3}(2,4,4,3,4,4,3)$, see Examples 4.6.

(\$) It was the surprising and unexpected thing for the author to find that even though $\mu$ and $\eta$ are partitions, the above inequality

$$
J_{\lambda \mu \eta}(q) \leq K_{\lambda \mu \eta}(q)
$$

may be wrong. For example, take $\lambda=(2,2,2,1,1)$ and $\mu=\eta=\left(1^{8}\right)$. Then

$$
\begin{aligned}
K_{\lambda \mu \eta}(q) & =q^{3}(1,1,2, \mathbf{2}, 3,3,4,3,3,2,2,1,1), \text { but } J_{\lambda \mu \eta}(q) \\
& =q^{3}(1,1,2, \mathbf{3}, 2,2,2,1,1,1,1,1,1) .
\end{aligned}
$$

Furthermore, one can show that $P_{(2,2,2,1,1),\left(1^{8}\right),\left(1^{8}\right)}(q, t)=$

$$
1+q^{6}(-1,1,2,2,2,1,1) t+\cdots+q^{117}(1,1,2,2,2,1,1,-1) t^{13}+q^{130} t^{14},
$$

see Section 5.4 for more details about the polynomials $P_{\left(2^{k}, 1^{n}\right),\left(1^{2 k+n}\right),\left(1^{2 k+n}\right)}$ $(q, t)$.

( However, we expect that if $\mu$ is a partition, then the initial and the leading terms of the polynomials $J_{\lambda \mu \eta}(q)$ and $K_{\lambda \mu \eta}(q)$ are the same.

( Moreover, we expect that if $\mu$ is an arbitrary composition, then $j_{\max }:=\max \left\{j \mid j \in J_{\lambda \mu \eta}\right\}=c(\lambda, \mu \| \eta)$ and $\#\left\{j \in J_{\lambda \mu \eta} \mid j=j_{\max }\right\} \leq$ $d(\lambda, \mu \| \eta)$, see Section 6.4, Rationality conjecture, for more detailed statements.

Examples 4.23. (1) Take $\lambda^{(1)}=(3,2,1), \lambda^{(2)}=(2,2), \mu^{(1)}=\left(1^{6}\right)$, $\mu^{(2)}=\left(1^{4}\right)$ and $\eta=\left(1^{6}\right)$. Then one can check that

$$
Q(q, x, y)=Q_{(3,2,1),\left(1^{6}\right),\left(1^{6}\right)}(q, x) Q_{(2,2),\left(1^{4}\right),\left(1^{4}\right)}(q, y)\left(1-q^{7} x y\right)\left(1-q^{8} x y\right),
$$

where $Q_{(3,2,1),\left(1^{6}\right),\left(1^{6}\right)}(q, x)=\left(1-q^{5} x\right) \prod_{j=4}^{11}\left(1-q^{j} x\right)$, see Example $4.20,(i i)$, and 
$Q_{(2,2),\left(1^{4}\right),\left(1^{4}\right)}(q, y)=\left(1-q^{2} y\right)\left(1-q^{4} y\right), P_{(2,2),\left(1^{4}\right),\left(1^{4}\right)}(q, y)=1$.

The expression for $P(q, x, y)$ is rather long, so we give here only the formula for its value at $q=1$. Namely,

$$
\begin{aligned}
& P(1, x, y)=\left[1+8 x+35 x^{2}+32 x^{3}+9 x^{4}+\left(6 x-44 x^{2}-118 x^{3}-81 x^{4}-18 x^{5}\right) y\right. \\
& \left.+\left(-3 x^{2}+40 x^{3}+143 x^{4}+66 x^{5}+9 x^{6}\right) y^{2}-\left(16 x^{4}+48 x^{5}+21 x^{6}\right) y^{3}\right](1-x) .
\end{aligned}
$$

Let us remark that in our case $Q(1, x, y)=(1-x)^{9}(1-y)^{2}(1-x y)^{2}$, and because of the well-known identity

$$
\left(1-x_{1} \ldots x_{k}\right)^{-1} \prod_{j=1}^{k}\left(1-x_{j}\right)^{-1}=\sum_{\left(n_{1}, \ldots, n_{k}\right) \in \mathbb{Z}_{\geq 0}^{k}} \min \left(n_{1}, \ldots, n_{k}\right) x_{1}^{n_{1}} \ldots x_{k}^{n_{k}},
$$

this example shows that the Kostka number $K_{n(3,2,1)+m(2,2),\left(n^{6}\right)+\left(m^{4}\right)}(1)$ considered as a function of $n$ and $m$ on the set $\left\{(n, m) \in \mathbb{Z}_{\geq 0}^{2}\right\}$, has at least two different polynomiality region, namely, "the dominant chamber" $\mathcal{N}_{2}=\{(n, m) \mid$ $n \geq m\}$ and that $\{(n, m) \mid n \leq m\}$. Moreover, since

$$
K_{n(3,2,1)+m(2,2),\left(n^{6}\right)+\left(m^{4}\right)}(1)=c_{n(5,4,3,2,1)+m(3,2,1), n(3,2,1)+m(2,2)}^{n(6,5,4,3,2,1)+m(4,3,2,1)},
$$

we see that if

$$
\lambda^{(1)}=(3,2,1), \lambda^{(2)}=(2,2), \mu^{(1)}=(5,4,3,2,1), \mu^{(2)}=(3,2,1), \nu^{(1)}=
$$
$(6,5,4,3,2,1)$ and $\nu^{(2)}=(4,3,2,1)$, then

(\$) the Littlewood-Richardson number $c_{n \lambda^{(1)}+m \lambda^{(2)}, n \mu^{(1)}+m \mu^{(2)}}^{n \nu^{(1)}+m \nu^{(2)}}$ considered as a function of $n$ and $m$ on the set $\left\{(n, m) \in \mathbb{Z}_{\geq 0}^{2}\right\}$, has the same (at least) two different polynomiality regions.

(2) Now take $\lambda^{(1)}=(3,2,1), \lambda^{(2)}=(2,2,1), \mu^{(1)}=\left(1^{6}\right), \mu^{(2)}=\left(1^{5}\right)$ and $\eta=\left(1^{6}\right)$. Then one can check that

$$
Q(q, x, y)=Q_{(3,2,1),\left(1^{6}\right),\left(1^{6}\right)}(q, x) Q_{(2,2,1),\left(1^{5}\right),\left(1^{5}\right)}(q, y)
$$

where $Q_{(2,2,1),\left(1^{5}\right),\left(1^{5}\right)}(q, y)=\left(1-q^{2} y\right)\left(1-q^{3} y\right)\left(1-q^{4} y\right)\left(1-q^{5} y\right)\left(1-q^{6} y\right)$.

Therefore, in this case the function $(n, m) \rightarrow K_{n(3,2,1)+m(2,2,1), n\left(1^{6}\right)+m\left(1^{5}\right), \eta}$ (1) is a polynomial function in $n$ and $m$ on the whole set $\left\{(n, m) \in \mathbb{Z}_{\geq 0}^{2}\right\}$.

It seems interesting to compare the above-described examples with the following result by P. McMullen [54]:

Let $\Delta_{1}, \ldots, \Delta_{k} \subset \mathbb{R}^{d}$ be integer convex polytopes, and $t_{1}, \ldots, t_{k} \in \mathbb{N}^{k}$. Given any integer polytope $\Gamma \subset \mathbb{R}^{d}$, denote by $N(\Gamma):=\#\left(\Gamma \cap \mathbb{Z}^{d}\right)$.

(\$)Mixed lattice point enumerator theorem (P. McMullen, [54]) 
$N\left(t_{1} \Delta_{1}+\cdots+t_{k} \Delta_{k}\right)$ is a polynomial in $t_{1}, \ldots, t_{k}$ with rational coefficients of total degree at most $d$. Moreover, the terms of degree $d$ are given by $\operatorname{Vol}\left(t_{1} \Delta_{1}+\cdots+t_{k} \Delta_{k}\right)$, the so-called mixed volume of the polytopes $\Delta_{1}, \ldots, \Delta_{k}$.

In other words, the generating function $\sum_{\left(n_{1}, \ldots, n_{k}\right) \in \mathbb{Z}_{\geq 0}^{k}} N\left(n_{1} \Delta_{1}+\cdots+\right.$ $\left.n_{k} \Delta_{k}\right) x^{n_{1}} \ldots x_{k}^{n_{k}}$ is a rational function in $x_{1}, \ldots, x_{k}$ with the (irredundant) denominator of the form $\prod_{j=1}^{k}\left(1-x_{j}\right)^{a_{j}}$ for some non-negative integers $a_{1}, \ldots, a_{k}$.

Remark 4.24 (Parabolic Kostka number $K_{\lambda \mu \eta}(1)$ as a function of $\lambda$ and $\mu$ ).

Let $\eta$ be a composition, $l(\eta)=p$. It follows from Theorem 3.25 that on the set

$$
Z_{\eta}=\left\{(\lambda, \mu) \in \mathbb{Z}_{\geq 0}^{n} \times \mathbb{Z}_{\geq 0}^{n} \mid \lambda_{1} \geq \lambda_{2} \geq \cdots \geq \lambda_{n}, \quad \lambda-\mu \in Y_{\eta}\right\}
$$

the function $(\lambda, \mu) \longrightarrow K_{\lambda \mu \eta}(1)$ is a continuous piecewise polynomial function $\mathcal{K}_{\eta}(\lambda, \mu)$ in $\lambda_{1}, \ldots, \lambda_{n}, \mu_{1}, \ldots, \mu_{n}$ of degree $\sum_{1 \leq i<j \leq p} \eta_{i} \eta_{j}-n+1$.

It is a challenge problem to describe the polynomiality domains of the function $(\lambda, \mu) \longrightarrow K_{\lambda \mu \eta}(1)$, and find the corresponding polynomials $\mathcal{K}_{\eta}(\lambda, \mu)$. In the case $\eta=\left(1^{n}\right)$ a partial solution to this problem has been done by B.V. Lidskii [51]. To the best of our knowledge, if $n \geq 4$, an explicit description of the polynomiality domains of the function $(\lambda, \mu) \longrightarrow K_{\lambda \mu \eta}(1)$ is not known.

Examples 4.25. (i) Take $n=3$, so that $\lambda=\left(\lambda_{1} \geq \lambda_{2} \geq \lambda_{3} \geq 0\right)$ and $\mu=\left(\mu_{1}, \mu_{2}, \mu_{3}\right)$. If $\mu$ is a partition, then

$$
K_{\lambda, \mu}(q)=q^{a(\lambda, \mu)}\left[\begin{array}{c}
N_{\lambda, \mu}+1 \\
1
\end{array}\right]_{q}
$$

where

$$
a(\lambda, \mu)=\max \left\{\lambda_{1}-\mu_{1}, \lambda_{1}+\lambda_{2}-\mu_{1}-\mu_{2}, \lambda_{1}+2 \lambda_{2}-2 \mu_{1}-\mu_{2}, 2 \lambda_{1}+\lambda_{3}-\right.
$$
$\left.2 \mu_{1}-\mu_{2}\right\}$

$N_{\lambda \mu}=\min \left\{\lambda_{1}-\lambda_{2}, \lambda_{2}-\lambda_{3}, \lambda_{1}-\mu_{1}, \lambda_{1}+\lambda_{2}-\mu_{1}-\mu_{2}\right\}$.

(\$) In particular, we see that $a(\lambda, \mu)$ is a homogeneous piecewise linear function in $\lambda_{1}, \lambda_{2}, \lambda_{3}$ and $\mu_{1}, \mu_{2}$.

Now let us define "the dominant chamber"

$$
Z_{\left(1^{3}\right)}^{++}=\left\{(\lambda, \mu) \in Z_{\left(1^{3}\right)} \mid \lambda_{3} \leq \mu_{2} \leq \lambda_{2} \leq \mu_{1} \leq \lambda_{1}, \lambda_{1}-\lambda_{2}+\lambda_{3} \leq \mu_{1}\right\}
$$

If $\mu$ is a partition, then

$$
\left.K_{\lambda \mu}(q)\right|_{Z_{\left(1^{3}\right)}^{++}}=K_{\Phi\left(1^{3}\right)}(\lambda-\mu \mid q)=q^{\lambda_{1}+\lambda_{2}-\mu_{1}-\mu_{2}}\left[\begin{array}{c}
\lambda_{1}-\mu_{1}+1 \\
1
\end{array}\right]_{q}
$$


(a) One can check that the domain $Z_{\left(1^{3}\right)}^{++}$is the maximal one among domains $D$ such that $\left.\mathcal{K}_{\left(1^{3}\right)}(\lambda, \mu)\right|_{D}=1+\lambda_{1}-\mu_{1}$.

(ii) Take $n=4$. In this case we don't have a complete description of the polynomiality domains of the function $(\lambda, \mu) \longrightarrow K_{\lambda \mu \eta}(1)$. Instead, we are going to describe "the dominant chamber" $Z_{\eta}^{++}$for the latter function, i.e. the maximal domain $D$ in the set

$Z_{\eta}^{+}:=\left\{(\lambda, \mu) \in Z_{\eta} \mid \lambda-\mu \in Y_{\eta}^{+}\right\}$such that $\left.K_{\lambda \mu \eta}(1)\right|_{D}=\left.\mathcal{K}_{\eta}(\lambda, \mu)\right|_{D}=$ $K_{\Phi(\eta)}(\lambda-\mu)$.

Proposition 4.26. Assume that $\eta=\left(1^{4}\right)$, and consider the sets

$$
\begin{gathered}
W_{4}^{(1)}=\left\{(\lambda, \mu) \in Z_{\left(1^{4}\right)}^{+} \mid \mu_{i} \geq m_{i+1}, i=1,2,3 ; 2 \mu_{2} \geq \lambda_{2}+\lambda_{3}\right\}, \text { and } \\
W_{4}^{(2)}=\left\{(\lambda, \mu) \in Z_{\left(1^{4}\right)}^{+} \mid \mu_{i} \geq m_{i+1}, i=1,2,3\right. \\
\left.2 \mu_{2} \leq \lambda_{2}+\lambda_{3}, \quad \lambda_{1}+\lambda_{3} \leq \mu_{1}+\mu_{2}\right\}
\end{gathered}
$$

Then

$$
\begin{aligned}
& \left.\mathcal{K}_{\left(1^{4}\right)}(\lambda, \mu)\right|_{W_{4}^{(1)}}=K_{\Phi\left(1^{4}\right)}(\lambda-\mu)-\left(\begin{array}{c}
\max \left(\lambda_{1}+\lambda_{3}-\mu_{1}-\mu_{2}, 0\right)+2 \\
3
\end{array}\right), \\
& \left.\mathcal{K}_{\left(1^{4}\right)}(\lambda, \mu)\right|_{W_{4}^{(2)}}=K_{\Phi\left(1^{4}\right)}(\lambda-\mu) .
\end{aligned}
$$

Proposition 4.27. We have

$$
Z_{\left(1^{4}\right)}^{++}=\left\{(\lambda, \mu) \in Z_{\left(1^{4}\right)} \mid \mu_{i} \geq m_{i+1}, i=1,2,3 ; \quad \lambda_{1}+\lambda_{3} \leq \mu_{1}+\mu_{2}\right\},
$$

and furthermore, $\left.K_{\lambda \mu}(q)\right|_{Z_{\left(1^{4}\right)}^{++}}=K_{\Phi\left(1^{4}\right)}(\lambda-\mu \mid q)$.

Problem 4.28. Describe explicitly "the dominant chamber" $Z_{\eta}^{++}$in general case.

At the end of this Remark we would like to say a few words about the Littlewood-Richardson numbers $c_{\lambda, \mu}^{\nu}$ considered as a function of $\lambda, \mu$ and $\nu$. To start with, let us consider the following set:

$$
\begin{gathered}
\mathcal{Z}_{n}:=\left\{(\lambda, \mu, \nu) \in \mathbb{Z}_{\geq 0}^{3 n} \mid \lambda_{1} \geq \cdots \geq \lambda_{n}, \mu_{1} \geq \cdots \geq \mu_{n},\right. \\
\left.\nu_{1} \geq \cdots \geq \nu_{n},|\lambda|+|\mu|=|\nu|\right\} .
\end{gathered}
$$

The next Proposition is an easy corollary of Theorem 3.25.

Proposition 4.29. The Littlewood-Richardson number $c_{\lambda, \mu}^{\nu}$ is a continuous piecewise polynomial function in $\lambda_{1}, \ldots, \lambda_{n}, \mu_{1}, \ldots, \mu_{n}, \nu_{1}, \ldots, \nu_{n}$ on the set $\mathcal{Z}_{n}$. 
Problem 4.30. Describe "the dominant chamber" for the function $(\lambda, \mu, \nu) \rightarrow c_{\lambda, \mu}^{\nu}$, i.e. the maximal domain $\mathcal{D}_{n} \subset \mathcal{Z}_{n}$ such that the restriction $\left.c_{\lambda, \mu}^{\nu}\right|_{\mathcal{D}_{n}}$ is a polynomial with non-negative rational coefficients.

Problem 4.31. Generalize the results obtained by B.V. Lidskii [51] for the function $(\lambda, \mu) \rightarrow K_{\lambda \mu}(1)$, to the case of the function $(\lambda, \mu, \nu)$ $\rightarrow c_{\lambda, \mu}^{\nu}$.

Remark 4.32. It is not difficult to see that Rationality Theorems 4.14 and 4.17, Polynomiality Theorem (Corollary 4.15) and Corollary 4.18, are still valid for the level $l$ restricted parabolic Kostka numbers $K_{\lambda \mu \eta}^{(l)}(1)$ and the level $l$ restricted parabolic Kostka polynomials $K_{\lambda \mu \eta}^{(l)}(q)$. Remember that the latter can be defined as follows

$$
K_{\lambda \mu \eta}^{(l)}(q)=\sum_{w \in \Sigma_{n, l}}(-1)^{l(w)} K_{\lambda, w \circ \mu, \eta}(q) .
$$

See Section 2.6 for a explanation of notation we have used.

Remark 4.33. In Section 4 we have studied a behavior of the parabolic Kostka polynomials $K_{n \lambda, n \mu, \eta}(q)$ as a function of $n$. We always have assumed that a composition $\eta$ is fixed. Here we would like to discuss briefly what happens if a composition $\eta$ is also varied. A naive way to vary $\eta$, say to consider $n \eta$, gives rise to a trivial result. We suggest the following way. In order to start, we need one definition, namely, let $\mu=\left(\mu_{1}, \mu_{2}, \ldots\right)$ be a composition. Define

$$
\mu^{\langle n\rangle}=(\underbrace{\mu_{1}, \ldots, \mu_{1}}_{n}, \underbrace{\mu_{2}, \ldots, \mu_{2}}_{n}, \ldots) .
$$

Let us remark that $(n \mu)^{\prime}=\mu^{\langle n\rangle}$.

Theorem 4.34. There exists the limit

$$
\lim _{n \rightarrow \infty} q^{c\left(n \lambda, \mu^{\langle n\rangle} \| \eta^{\langle n\rangle}\right)} K_{n \lambda, \mu}^{\langle n\rangle, \eta^{\langle n\rangle}}\left(q^{-1}\right):=X_{\lambda \mu \eta}(q),
$$

which is a formal power series in $q$.

( $)$ We expect that if $\mu$ is a partition, then the formal power series $X_{\lambda \mu \eta}(q)$ has non-negative integer coefficients. For example,

$$
X_{(3,2,1),\left(1^{6}\right),\left(1^{6}\right)}(q)=\prod_{n \geq 1}\left(1-q^{n}\right)^{-2} .
$$


However, we would like to remark that the limit

$$
\lim _{n \rightarrow \infty} q^{-a\left(n \lambda, \mu^{\langle n\rangle} \| \eta^{\langle n\rangle}\right)} K_{n \lambda, \mu^{\langle n\rangle}, \eta^{\langle n\rangle}}(q)
$$

does not exist in general.

Finally, it looks as an interesting problem to study the generating functions

$$
\sum_{n \geq 0} K_{\lambda^{\langle n\rangle}, \mu^{\langle n\rangle}, \eta^{\langle n\rangle}}(q) t^{n} \text { and } \sum_{n \geq 0} K_{\lambda^{\langle n\rangle}, \mu^{\langle n\rangle}, n \eta}(q) t^{n}
$$

( We expect that the latter generating function is a rational function in $q$ and $t$.

Remark 4.35 (Parabolic Hall-Littlewood polynomials $Q_{\mu, \eta}(X ; q)$ ).

Let $\mu$ and $\eta$ be compositions such that $|\eta| \geq l l(\mu)$, and $X=\left(x_{1}, \ldots, x_{n}\right)$ be the set of variables. Define the modified parabolic Hall-Littlewood polynomials $Q_{\mu, \eta}^{\prime}(X ; q)$ as follows:

$$
Q_{\mu, \eta}^{\prime}(X ; q)=\sum_{\lambda} K_{\lambda \mu \eta}(q) s_{\lambda}(X)
$$

and the parabolic Hall-Littlewood polynomial $Q_{\mu, \eta}(X ; q)$ using the plethystic transformation:

$$
Q_{\mu, \eta}(X ; q)=Q_{\mu, \eta}^{\prime}(X(1-q) ; q)
$$

Theorem 4.36 (Rationality theorem for parabolic Hall-Littlewood polynomials).

The generating function $\sum_{n \geq 0} Q_{n \mu, \eta}(X ; q) t^{n}$ is a rational function in $q, t$ and $X$.

In particular, the generating function $\sum_{n \geq 0} s_{n \lambda}(X) t^{n}$ for Schur functions is a rational function in $t$ and $X$.

On the other hand, the generating function for the double Kostka polynomials

$$
Z_{\lambda, \mu}(q, t, x):=\sum_{n \geq 0} K_{n \lambda, n \mu}(q, t) x^{n}
$$

is a formal power series in $q, t$ and $x$ which, in general, cannot be equal to any rational function. 


\section{§5. Parabolic Kostka Polynomials: Examples}

\section{§5.1. Parabolic Kostka and Kostka-Foulkes polynomials}

\section{$1^{0}$ [Kostka-Foulkes and parabolic Kostka polynomials]}

Let $\lambda$ be a partition and $R=\left(R_{1}, R_{2}, \ldots, R_{r}\right)$ be a dominant sequence of rectangular shape partitions.

(i) Let $R_{a}$ be the single row $\left(\mu_{a}\right)$ for all $a$, and $\mu:=\left(\mu_{1}, \mu_{2}, \ldots\right)$ is a partition of length at most $n$. Then

$$
K_{\lambda R}(q)=K_{\lambda \mu}(q),
$$

i.e. $K_{\lambda R}(q)$ coincides with the Kostka-Foulkes polynomial $K_{\lambda \mu}(q)$.

(ii) Let $R_{a}$ be the single column $\left(1^{\eta_{a}}\right)$ for all $a$, and $\eta=\left(\eta_{1}, \eta_{2}, \ldots\right)$. Then

$$
K_{\lambda R}(q)=\bar{K}_{\lambda^{\prime} \eta^{+}}(q)
$$

the cocharge Kostka-Foulkes polynomial, where $\lambda^{\prime}$ is the conjugate of the partition $\lambda$, and $\eta^{+}$is the partition obtained by sorting the parts of $\eta$ into weakly decreasing order. Formula (5.40) follows from that (5.39) and Duality Theorem for parabolic Kostka polynomials.

$2^{0}$ [Parabolic Kostka polynomials and Kostant partition function]

Let $\gamma \in \mathbb{Z}^{n},|\gamma|=0, N$ be an integer such that $N+n\left(\gamma_{i}-\gamma_{i+1}\right) \geq 0$ for all $1 \leq i \leq n$, where we put $\gamma_{n+1}=0$. Consider partitions $\lambda_{N}=N(n, n-$ $1, \ldots, 2,1)+\gamma, \mu_{N}=N(n, n-1, \ldots, 2,1)$ and composition $\eta,|\eta|=n$. Then

$$
K_{\Phi(\eta)}(\gamma \mid q)=K_{\lambda_{N}, \mu_{N}, \eta}(q)
$$

$3^{0}$ [ Skew Kostka-Foulkes and parabolic Kostka polynomials]

Let $\lambda \supset \mu$ be partitions, $l(\lambda)=n$, and $\boldsymbol{\nu}$ be a sequence of partitions.

Define $\left.\mu_{0}=(\mu, \underbrace{0, \ldots, 0}_{n-l(\mu)})\right)$. Then

$$
K_{\lambda \backslash \mu, \boldsymbol{\nu}}(q) \stackrel{\bullet}{=} K_{\lambda,\left(\mu_{0}, \boldsymbol{\nu}\right)}(q)
$$

If $\mu$ is a rectangular shape partition and $R$ is a dominant sequence of rectangular shape partitions, then

$$
K_{\lambda \backslash \mu, R}(q) \doteq K_{\lambda,(\mu, R)^{+}}(q) \doteq K_{\lambda,\left(\mu_{0}, R\right)}(q),
$$

where $(\mu, R)^{+}$denotes a dominant rearrangement of the sequence of rectangular shape partitions $(\mu, R)$. 
Example 5.1. Let $\lambda$ and $\mu$ be partitions, $\mu \subset \lambda,|\lambda \backslash \mu|=N$, and the complement $\lambda \backslash \mu=\coprod \lambda^{(i)}$ is a disjoint union of partitions $\lambda^{(i)},\left|\lambda^{(i)}\right|=n_{i}$, $i=1, \ldots, s$. Then $\quad K_{\lambda \backslash \mu,\left(1^{N}\right)}(q)=$

$$
\begin{aligned}
q^{N} & \prod_{i=1}^{s} K_{\lambda^{(i)},\left(1^{n^{i}}\right)}(q)\left[\begin{array}{c}
N \\
n_{1}, \ldots, n_{s}
\end{array}\right]_{q} \\
= & q^{N+\sum n\left(\lambda^{(i) \prime}\right)}[N] ! / \prod_{i=1}^{s} H_{\lambda^{(i)}}(q)=K_{\lambda,\left(\mu, 1^{N}\right)}(q),
\end{aligned}
$$

where for any partition $\lambda, H_{\lambda}(q)$ denotes the hook polynomial corresponding to $\lambda$, see e.g. [53], p.45.

In particular, if $n \geq m$, then $K_{(n, m),\left(n, 1^{m}\right)}(q) \doteq\left[\begin{array}{c}n \\ m\end{array}\right]_{q}$.

Example 5.2. Let $\lambda$ and $\mu$ be partitions, $\mu \subset \lambda,|\lambda \backslash \mu|=l$, and the complement $\lambda \backslash \mu=\coprod \lambda^{(i)}$ is a disjoint union of partitions $\lambda^{(i)},\left|\lambda^{(i)}\right|=n_{i}$, $i=1, \ldots, s$. Define partitions $\widetilde{\lambda}=(N l+|\mu|, \lambda)$ and $\widetilde{\mu}=(l, \mu)$. Then

$$
K_{\widetilde{\lambda} \backslash \widetilde{\mu},\left(l^{N}\right)} \doteq \prod_{i=1}^{s}\left[\begin{array}{c}
N \\
\lambda^{(i) \prime}
\end{array}\right]_{q} .
$$

We would like to emphasize that, in general, the parabolic Kostka polynomial $K_{\lambda,(\mu, \boldsymbol{\nu})}(q)$ is different from the skew Kostka-Foulkes polynomial $K_{\lambda \backslash \mu, \boldsymbol{\nu}}$ $(q)$.

For example, take $\lambda=(2,2), \mu=(1)$ and $R=(3)$. Then $K_{\lambda \backslash \mu, R}(q)=$ $K_{\lambda,\left(\mu_{0}, R\right)}(q)=0$, but $K_{\lambda,(\mu, R)}(q)=-1+q$.

\section{$4^{0}$ [Principal specialization of skew Schur functions]}

Let $\lambda \supset \mu$ be partitions, $|\lambda \backslash \mu|=r$, and $N \geq 1$ be an integer number. Then

$$
s_{\lambda \backslash \mu}\left(1, q, \ldots, q^{N-1}\right) \doteq K_{(N r, \lambda) \backslash(r, \mu),(\underbrace{r, \ldots, r}_{N})}(q) .
$$

If $\mu=\emptyset$, then

$$
s_{\lambda}\left(1, q, \ldots, q^{N-1}\right) \doteq K_{(N|\lambda|, \lambda),(\underbrace{r, \ldots, r}_{N+1})}(q) \doteq\left[\begin{array}{c}
N \\
\lambda^{\prime}
\end{array}\right]_{q} .
$$

The second equality in (5.42) together with the fermionic formula (5.44) for the Kostka-Foulkes polynomials, is a crucial step in a combinatorial proof of unimodality of the generalized $q$-Gaussian coefficients $\left[\begin{array}{c}N \\ \lambda\end{array}\right]_{q}$, see [30] for details. 
Example 5.3 (A q-analogue of Merris' conjecture, cf [56], [33]).

Let $\lambda$ and $\mu$ be partitions such that $\lambda \geq \lambda^{\prime}$ with respect to the dominance partial ordering, see Section 2.1. Then

- $a(\lambda, \mu) \geq a\left(\lambda^{\prime}, \mu\right)$.

- (q-Analogue of Merris' conjecture)

$$
K_{\lambda, \mu}(q) \geq q^{n\left(\lambda^{\prime}\right)-n(\lambda)} K_{\lambda^{\prime}, \mu}(q) .
$$

Question. If the above inequality is true, what is the case of equality?

For example, the equality holds for any partition $\lambda$ if $\mu=\left(1^{n}\right)$. It's not difficult to see that the equality also holds if

$$
\lambda=\left(n, m, 1^{n-2}\right) \quad \text { and } \quad \mu=\left(2^{n-1+[m / 2]}, \varepsilon_{m}\right)
$$

for some positive integers $n \geq m$ and $m \leq 4$. Here $\varepsilon_{m}=0$ or 1 according to the parity of $m$.

Question. Could it be true that these two examples are the only infinite families of partitions $\lambda$ and $\mu$ such that $\lambda \gtreqless \lambda^{\prime}$ and $K_{\lambda, \mu}=K_{\lambda^{\prime}, \mu}$ ?

( Moreover, we expect that the difference

$$
K_{\lambda, \mu}(q)-q^{n\left(\lambda^{\prime}\right)-n(\lambda)} K_{\lambda^{\prime}, \mu}(q)
$$

is a unimodal polynomial (with non-negative integer coefficients). In particular,

( we expect that if $\lambda \geq \lambda^{\prime}$, then for any positive integer $N$ the difference

$$
\left[\begin{array}{c}
N \\
\lambda
\end{array}\right]_{q}-q^{n\left(\lambda^{\prime}\right)-n(\lambda)}\left[\begin{array}{l}
N \\
\lambda^{\prime}
\end{array}\right]_{q}
$$

is a unimodal polynomial (with non-negative integer coefficients).

$5^{0}$ [ Fermionic formula for polynomials $\left.K_{\lambda, R}(q)\right]$

Let $\lambda$ be a partition and $R=\left(\left(\mu_{a}^{\eta_{a}}\right)\right)_{a=1}^{p}$ be a sequence of rectangular shape partitions such that

$$
|\lambda|=\sum_{a}\left|R_{a}\right|=\sum_{a} \mu_{a} \eta_{a} .
$$

Definition 5.4. A configuration of type $(\lambda ; R)$ is a sequence of partitions $\nu=\left(\nu^{(1)}, \nu^{(2)}, \ldots\right)$ such that

$$
\left|\nu^{(k)}\right|=\sum_{j>k} \lambda_{j}-\sum_{a \geq 1} \mu_{a} \max \left(\eta_{a}-k, 0\right)=-\sum_{j \leq k} \lambda_{j}+\sum_{a \geq 1} \mu_{a} \min \left(k, \eta_{a}\right)
$$

for each $k \geq 1$. 
Note that if $k \geq l(\lambda)$ and $k \geq \eta_{a}$ for all $a$, then $\nu^{(k)}$ is empty. So that each configuration contains only a finite number of partitions. In the sequel (except Corollary 5.7) we make the convention that $\nu^{(0)}$ is the empty partition.

For a partition $\mu$ define the number $Q_{n}(\mu)=\mu_{1}^{\prime}+\cdots+\mu_{n}^{\prime}$, which is equal to the number of cells in the first $n$ columns of $\mu$.

The vacancy numbers $P_{n}^{(k)}(\nu):=P_{n}^{(k)}(\nu ; R)$ of a configuration $\nu$ of type $(\lambda ; R)$ are defined by

$$
P_{n}^{(k)}(\nu)=Q_{n}\left(\nu^{(k-1)}\right)-2 Q_{n}\left(\nu^{(k)}\right)+Q_{n}\left(\nu^{(k+1)}\right)+\sum_{a \geq 1} \min \left(\mu_{a}, n\right) \delta_{\eta_{a}, k}
$$

for $k, n \geq 1$, where $\delta_{i, j}$ is the Kronecker delta.

Definition 5.5. A configuration $\nu$ of type $(\lambda ; R)$ is called admissible, if

$$
P_{n}^{(k)}(\nu ; R) \geq 0 \text { for all } k, n \geq 1 .
$$

We denote by $C(\lambda ; R)$ the set of all admissible configurations of type $(\lambda ; R)$, and call a vacancy number $P_{n}^{(k)}(\nu ; R)$ essential, if $m_{n}\left(\nu^{(k)}\right)>0$.

Finally, for a configuration $\nu$ of type $(\lambda ; R)$ let us define its charge

$$
c(\nu)=\sum_{k, n \geq 1}\left(\begin{array}{c}
\alpha_{n}^{(k-1)}-\alpha_{n}^{(k)}+\sum_{a} \theta\left(\eta_{a}-k\right) \theta\left(\mu_{a}-n\right) \\
2
\end{array}\right),
$$

and cocharge

$$
\bar{c}(\nu)=\sum_{k, n \geq 1}\left(\begin{array}{c}
\alpha_{n}^{(k-1)}-\alpha_{n}^{(k)} \\
2
\end{array}\right),
$$

where $\alpha_{n}^{(k)}=\left(\nu^{(k)}\right)_{n}^{\prime}$ denotes the size of the $n$-th column of the $k$-th partition $\nu^{(k)}$ of the configuration $\nu$; thus, $\alpha_{n}^{(0)}=0, \forall n \geq 1$. For any real number $x \in \mathbb{R}$ we put $\theta(x)=1$, if $x \geq 0$, and $\theta(x)=0$, if $x<0$.

Theorem 5.6 (Fermionic formula for parabolic Kostka polynomials $[33,42])$.

Let $\lambda$ be a partition and $R$ be a dominant sequence of rectangular shape partitions. Then

$$
K_{\lambda R}(q)=\sum_{\nu} q^{c(\nu)} \prod_{k, n \geq 1}\left[\begin{array}{c}
P_{n}^{(k)}(\nu ; R)+m_{n}\left(\nu^{(k)}\right) \\
m_{n}\left(\nu^{(k)}\right)
\end{array}\right]_{q}
$$

summed over all admissible configurations $\nu$ of type $(\lambda ; R) ; m_{n}(\lambda)$ denotes the number of parts of the partition $\lambda$ of size $n$. 
Corollary 5.7 (Fermionic formula for Kostka-Foulkes polynomials [29]). Let $\lambda$ and $\mu$ be partitions of the same size. Then

$$
K_{\lambda \mu}(q)=\sum_{\nu} q^{c(\nu)} \prod_{k, n \geq 1}\left[\begin{array}{c}
P_{n}^{(k)}(\nu, \mu)+m_{n}\left(\nu^{(k)}\right) \\
m_{n}\left(\nu^{(k)}\right)
\end{array}\right]_{q}
$$

summed over all sequences of partitions $\nu=\left\{\nu^{(1)}, \nu^{(2)}, \ldots\right\}$ such that

- $\left|\nu^{(k)}\right|=\sum_{j>k} \lambda_{j}, k=1,2, \ldots$;

- $P_{n}^{(k)}(\nu, \mu):=Q_{n}\left(\nu^{(k-1)}\right)-2 Q_{n}\left(\nu^{(k)}\right)+Q_{n}\left(\nu^{(k+1)}\right) \geq 0$ for all $k, n \geq 1$, where by definition we put $\nu^{(0)}=\mu$;

$$
\text { - } c(\nu):=\sum_{k, n \geq 1}\left(\begin{array}{c}
\left(\nu^{(k-1)}\right)_{n}^{\prime}-\left(\nu^{(k)}\right)_{n}^{\prime} \\
2
\end{array}\right) \text {. }
$$

\section{§5.2. Parabolic Kostka polynomials and Littlewood-Richardson numbers}

$\left(1^{0}\right)$ Let $\lambda, \mu, \nu$ be partitions, $|\nu|=|\lambda|+|\mu|, l(\lambda)=p, l(\mu)=s$. Consider partition

$$
\widetilde{\lambda}=\left(\lambda_{1}+\mu_{1}, \ldots, \lambda_{1}+\mu_{s}, \lambda_{1}, \lambda_{2}, \ldots, \lambda_{p}\right)
$$

and a dominant rearrangement $\widetilde{R}$ of the sequence of rectangular shape partitions $R=\left\{\nu \cup\left(\lambda_{1}^{s}\right)\right\}$. Then

$$
K_{\widetilde{\lambda}, \widetilde{R}}(q)=q^{a(\widetilde{\lambda}, \widetilde{R})}\left\{c_{\lambda \mu}^{\nu}+\cdots+q^{n(\nu)-n(\lambda)-n(\mu)}\right\},
$$

where $c_{\lambda \mu}^{\nu}$ denotes the Littlewood-Richardson number, i.e. $c_{\lambda \mu}^{\nu}=$ Mult $\left[V_{\nu}: V_{\lambda} \otimes V_{\mu}\right]$.

Furthermore, $a(\widetilde{\lambda}, \widetilde{R}) \geq \sum_{j \leq \lambda_{1}} \nu_{j}^{\prime}-|\lambda|$, and $a(\widetilde{\lambda}, \widetilde{R})=\sum_{j \leq \lambda_{1}} \nu_{j}^{\prime}-|\lambda|$ if and only if $c_{\lambda, \mu}^{\nu} \neq 0$.

In other words, if $a(\widetilde{\lambda}, \widetilde{R})=\sum_{j \leq \lambda_{1}} \nu_{j}^{\prime}-|\lambda|$, then $c_{\lambda, \mu}^{\nu} \neq 0$, and

(\$) the coefficient $b(\widetilde{\lambda}, \widetilde{R})$ is equal to the Littlewood-Richardson number $c_{\lambda \mu}^{\nu}=c_{\left(\lambda_{1}^{s}\right), \nu}^{\widetilde{1}}$.

(\$p) Moreover, $K_{\widetilde{\lambda}, \widetilde{R}}(1)$ is equal to the number $\#\left|T a b^{(2)}\left(\Lambda^{(2)}, \nu\right)\right|$ of semistandard domino tableaux of the shape $\Lambda^{(2)}$ and content $\nu$, where $\Lambda^{(2)}$ is a unique partition such that 
- $\quad 2$-core $\left(\Lambda^{(2)}\right)=\emptyset$

- $\quad 2$-quotient $\left(\Lambda^{(2)}\right)=(\lambda, \mu)$.

The partition $\Lambda^{(2)}:=\Lambda^{(2)}(\lambda, \mu)$ can be constructed, see e.g. [18], as follows: Take an integer $r \geq \max (l(\lambda), l(\mu))$, then

$$
\begin{aligned}
& \Lambda^{(2)}(\lambda, \mu)+(2 r, 2 r-1, \ldots, 2,1) \\
&=\left(2 \lambda_{1}+2 r-1, \ldots, 2 \lambda_{k}+2(r-k)+1, \ldots, 2 \lambda_{r}+1\right) \\
& \quad \times \cup\left(2 \mu_{1}+2 r, \ldots, 2 \mu_{j}+2(r-j), 2 \mu_{r}+2\right) .
\end{aligned}
$$

Remember, [53], p.6, that if $\lambda$ and $\mu$ are partitions, then $\lambda \cup \mu$ denotes the partition whose parts are those of $\lambda$ and $\mu$, arranged in descending order.

Example 5.8. Take $\lambda=\mu=(2,1)$ and $\nu=(3,2,1)$. Then $\widetilde{\lambda}=$ $(4,3,2,1), \widetilde{R}=(3,(2,2), 2,1)$ and $K_{\widetilde{\lambda}, \widetilde{R}}(q)=q^{2}(2,3,1)$. More generally,

$$
\sum_{n \geq 0} K_{n \widetilde{\lambda}, n \widetilde{R}}(q) t^{n}=\left(1-q^{8} t^{2}\right) /\left(1-q^{3} t\right)^{2}\left(1-q^{4} t\right)^{3}\left(1-q^{5} t\right)
$$

It is easy to see that $a(\widetilde{\lambda}, \widetilde{R})=3=|\nu|-|\mu|$ and $b(\widetilde{\lambda}, \widetilde{R})=2=c_{\lambda, \mu}^{\nu}$.

Furthermore, $\Lambda:=\Lambda^{(2)}=(4,4,2,2)$, and the spin polynomial [11], and the charge-spin polynomial $K_{\lambda \mu}^{\nu}(q, t)[35]$ are equal to:

$$
\begin{aligned}
& \sum_{T \in \operatorname{Tab}^{(2)}(\Lambda, \nu)} t^{\operatorname{spin}(T)}=t+3 t^{2}+2 t^{3}, K_{\lambda \mu}^{\nu}(q, t) \\
& =\sum_{T \in T a b^{(2)}(\Lambda, \nu)} q^{\operatorname{charge}(T)} t^{\operatorname{spin}(T)}=q^{3} t(1+q t)(1+t+q t) .
\end{aligned}
$$

Thus, $c_{\lambda, \mu}^{\nu}(t)=\left.K_{\lambda \mu}^{\nu}(q, t)\right|_{q^{3}}=t+t^{2}$, where $c_{\lambda, \mu}^{\nu}(t)$ denotes the $L L T$-analog of the $L R$-number $c_{\lambda, \mu}^{\nu}$.

$\left(2^{0}\right)$ More generally, let $\lambda \supset \mu$ be partitions such that the complement $\lambda \backslash \mu$ is a disjoint union of partitions $\lambda^{(1)}, \ldots, \lambda^{(p)}$, and $l(\mu)=m$. Let $\nu$ be a partition, define composition $\widetilde{\nu}=(\mu, \nu)$ and partition $\eta=\left(m, 1^{|\nu|}\right)$. Then

$$
K_{\lambda \widetilde{\nu} \eta}(q)=q^{a(\lambda, \mu, \nu)}\left(c_{\lambda^{(1)}, \ldots, \lambda(p)}^{\nu}+\cdots+q^{n(\nu)-n\left(\lambda^{(1)}\right)-\cdots-n\left(\lambda^{(p)}\right)}\right),
$$

where

$$
c_{\lambda^{(1)}, \ldots, \lambda^{(p)}}^{\nu}:=\operatorname{Mult}\left[V_{\nu}: V_{\lambda^{(1)}} \otimes \cdots \otimes V_{\lambda^{(p)}}\right]
$$

denotes the (multiple) Littlewood-Richardson coefficient, and $a(\lambda, \mu, \nu)$ $\in \mathbb{Z}_{\geq 0}$. 
(\$) Moreover, $K_{\lambda \widetilde{\nu} \eta}(1)=\#\left|\operatorname{Tab}^{(p)}\left(\Lambda^{(p)}, \nu\right)\right|$ is equal to the number of semistandard $p$-rim hook tableaux of shape $\Lambda^{(p)}$ and content $\nu$, where $\Lambda^{(p)}$ is a unique partition such that

- $p$-core $\left(\Lambda^{(p)}\right)=\emptyset$.

- $p$-quotient $\left(\Lambda^{(p)}\right)=\left(\lambda^{(1)}, \lambda^{(2)}, \ldots, \lambda^{(p)}\right)$.

Similar to the case $p=2$, the partition $\Lambda^{(p)}$ can be constructed as follows: Take an integer $r \geq \max \left(l\left(\lambda^{(1)}\right), \ldots, l\left(\lambda^{(p)}\right)\right)$, then

$$
\begin{aligned}
& \Lambda^{(p)}+(p r, p r-1, \ldots, 2,1)=\cup_{k=1}^{p}\left(p \lambda_{1}^{(k)}\right. \\
& \left.\quad+p(r-1)+k, \ldots, p \lambda_{j}^{(k)}+p(r-j)+k, \ldots, p \lambda_{r}^{(k)}+k\right) .
\end{aligned}
$$

We refer the reader to [53], Chapter I, Section 1, Example 8, for definitions of the $p$-core and $p$-quotient of a partition $\Lambda$, and [47] for the definition of semistandard $p$-rim hook tableaux (domino tableaux in the case $p=2$ ).

( ) Note also, that the order of parts in the definition of composition $\widetilde{\nu}$ is important.

$\left(3^{0}\right)$ Let $A=\Lambda \backslash \lambda$ and $B=M \backslash \mu$ be skew diagrams and $\nu$ be a partition. Define partitions

$$
\alpha=\left(\left(M_{1}^{\Lambda_{1}^{\prime}}\right)+\Lambda\right) * M, \quad \eta=\left(\Lambda_{1}^{\prime}+M_{1}^{\prime}, 1^{|\nu|}\right), \quad \gamma=\left(\left(M_{1}^{\Lambda_{1}^{\prime}}+\lambda\right) * \mu\right)
$$

and composition

$$
\beta=\left(\left(\left(M_{1}^{\Lambda_{1}^{\prime}}+\lambda\right) * \mu, 0^{M_{1}^{\prime}-\mu_{1}^{\prime}}\right) * \nu\right) .
$$

Assume that $|A|+|B|=|\nu|$, Then

(A) $K_{\alpha \beta \eta}(q)=q^{|\nu|} K_{\alpha \backslash \gamma, \nu}(q)=q^{|\nu|}\left\{c_{A, B}^{\nu}+\right.$ higher degree terms in $\left.q\right\}$.

Therefore, $a(\alpha, \beta \| \eta) \geq|\nu|$, and $a(\alpha, \beta \| \eta)=|\nu|$ if and only if $c_{A, B}^{\nu} \neq 0$. In this case

$$
b(\alpha, \beta \| \eta)=c_{A, B}^{\nu}=\left\langle s_{A} s_{B}, s_{\nu}\right\rangle,
$$

where $b(\alpha, \beta \| \eta)$ denotes the initial coefficient of the polynomial $K_{\alpha \beta \eta}(q)$, see Definition 6.1, $s_{A}$ and $s_{B}$ denote the skew Schur functions corresponding to the skew diagrams $A$ and $B$, and $\langle\bullet, \bullet\rangle$, denotes the scalar product (the so-called Redfield-Hall scalar product) on the ring of symmetric functions, see e.g. [53], Chapter I, Section 4.

We don't know any "nice" combinatorial interpretation of the numbers $K_{\alpha \beta \eta}(1)$ or $K_{\alpha \beta \eta}(-1)$.

For a nice combinatorial description of the numbers $c_{A, B}^{\nu}$ in terms of "pictures", see [73]. 
See also Section 6.8 for a slightly different exposition of connections between the Littlewood-Richardson numbers and the parabolic Kostka polynomials.

\section{§5.3. MacMahon polytope and rectangular Narayana numbers [35]}

Take $\lambda=(n+k, n, n-1, \ldots, 2)$ and $\mu=\lambda^{\prime}=\left(n, n, n-1, n-2, \ldots, 2,1^{k}\right)$. If $n \geq k \geq 1$, then for any positive integer $N$

- $a(N \lambda, N \mu)=(2 k-1) N$;

- $b(N \lambda, N \mu)=\operatorname{dim} V_{\left((n-k+1)^{k-1}\right)}^{\mathfrak{g} l(N+k-1)}=\prod_{i=1}^{k-1} \prod_{j=1}^{n-k+1} \frac{N+i+j-1}{i+j-1}$.

In other words, $b(N \lambda, N \mu)$ is equal to the number of (weak) plane partitions of rectangular shape $\left((n-k+1)^{k-1}\right)$ whose parts do not exceed $N$, see e.g. [53], [67]. It is well-known, see e.g. [35], [67], that the number $b(N \lambda, N \mu)$ is equal also to the number $i\left(\mathfrak{M}_{k-1, n-k+1} ; N\right)$ of rational points $\mathbf{x}$ in the MacMahon polytope $\mathfrak{M}_{k-1, n-k+1}$ such that the points $N \mathbf{x}$ have integer coordinates. The generating function for the numbers $b(n \lambda, n \mu)$ has the following form

$\sum_{n \geq 0} b(n \lambda, n \mu) t^{n}=\left(\sum_{j=0}^{(k-2)(n-k)} N(k-1, n-k+1 ; j) t^{j}\right) /(1-t)^{(k-1)(n-k+1)+1}$,

where $N(k, n ; j), 0 \leq j \leq(k-1)(n-1)$, denote the rectangular Narayana numbers. For definition of the rectangular Narayana numbers and the MacMahon polytope, see [35], Section 2, Exercise 1.

For the reader's convenience, we display the numbers $b(N \lambda, N \mu)$ for small values of $k$ and $N$.

If $k=1$, then $b(N \lambda, N \mu)=1$ for all integer numbers $N \geq 1$.

If $k=2$, then $b(N \lambda, N \mu)=\left(\begin{array}{c}N+n-1 \\ N\end{array}\right)$.

If $N=1$, then $b(\lambda, \mu)=\left(\begin{array}{c}n \\ k-1\end{array}\right)$.

If $N=2$, then $b(2 \lambda, 2 \mu)=\frac{1}{k}\left(\begin{array}{c}n \\ k-1\end{array}\right)\left(\begin{array}{c}n+1 \\ k-1\end{array}\right)$.

Thus, the number $b(2 \lambda, 2 \mu)$ is equal to the Narayana number $N_{k-1, n+1}$.

Note also, that

$$
b(N \lambda, N \mu)=K_{N\left(k, 1^{n-k+1}\right), N\left(1^{n}\right)}(1) .
$$


More generally, see e.g. [30],

$$
K_{N\left(k, 1^{n-k+1}\right), N\left(1^{n}\right)}(q)=q^{k N} \prod_{i=1}^{k-1} \prod_{j=1}^{n-k+1} \frac{1-q^{N+i+j-1}}{1-q^{i+j-1}}=q^{k N}\left[\begin{array}{c}
N \\
\alpha
\end{array}\right]_{q}
$$

where $\alpha$ is a rectangular shape partition $\left((k-1)^{n-k+1}\right)$.

In particular, $K_{N\left(k, 1^{n-k+1}\right), N\left(1^{n}\right)}(q)$ is a symmetric and unimodal polynomial in $q$.

This example and many others, suggests the following

Problem 5.9. Define a q-analog of the numbers $d(\lambda, \mu \| \eta)$, in particular the numbers $b(\lambda, R)$, which generalizes the q-analog of the LR-numbers introduced by A. Lascoux, B. Leclerc and J.-Y. Thibon, see e.g. [49].

\section{§5.4. Gelfand-Tsetlin's polytope $G T\left(\left(2^{k}, 1^{n}\right),\left(1^{2 k+n}\right)\right)$}

Let $\lambda=\left(2^{k}, 1^{n}\right), k>0$, be a two-column partition, and $\mu=\eta=$ $\left(1^{2 k+n}\right)$. In this Section we are going to study in more details the polynomials $P_{k, n}(q, t):=P_{\lambda \mu \eta}(q, t), P_{k, n}(t):=P_{\lambda \mu \eta}(1, t), J_{k, n}(q):=J_{\lambda \mu \eta}(q)$, as well as the Gelfand-Tsetlin polytope $G T_{k, n}:=G T(\lambda, \mu)$.

We refer the reader to $[12,35,38,67]$, vol.2, for the definition and basic properties of the Gelfand-Tsetlin polytope $G T(\lambda, \mu)$ corresponding to a partition $\lambda$ and composition $\mu$.

First of all, let us remember [35] the formula for the dimension of GelfandTsetlin's polytope $G T(\lambda, \mu)$, namely, if $\lambda$ and $\mu$ are partitions, $l(\lambda)=r, l(\mu)=$ $s$, then

$$
\operatorname{dim} G T(\lambda, \mu)=(r-1)(s-1)-\left(\begin{array}{l}
r \\
2
\end{array}\right)-\sum_{i=1}^{r}\left(\begin{array}{c}
\lambda_{i}^{\prime}-\lambda_{i+1}^{\prime} \\
2
\end{array}\right),
$$

where $\lambda_{i}^{\prime}:=\#\left\{j \mid \lambda_{j} \geq i\right\}$.

In particular, $\operatorname{dim} G T_{k, n}=n(2 k-1)+(k-1)^{2}$.

Proposition 5.10. (1) $\operatorname{deg}_{t} P_{k, n}(t)=\operatorname{dim} G T_{k, n}+1-k-n=(k-$ 1) $(2 n+k-2)$;

(2) $P_{k, n}(q, t)=(-1)^{a_{k, n}} q^{b_{k, n}} t^{c_{k, n}} P_{k, n}\left(q^{-1}, t^{-1}\right)$,

where $a_{k, n}=\left(\sum_{j \in J_{k, n}} j\right)-\operatorname{dim} G T_{k, n}-1$, and $b_{k, n}$, and $c_{k, n}$ are certain non-negative integers.

In particular, $P_{k, n}(t)$ is a symmetric polynomial (with non-negative coefficients). 
(\$) We will say that a polynomial $P(q, t)$ is a reciprocal one if it satisfies the following condition:

$$
P(q, t)=(-1)^{a} q^{b} t^{c} P\left(q^{-1}, t^{-1}\right)
$$

for some non-negative integers $a, b$ and $c$.

Examples 5.11. (i) Take $k=4, n=0$, then $\operatorname{dim} G T_{4,0}=9, K_{\lambda \mu}(q)=$ $J_{4,0}(q)=q^{4}(1,0,1,1,2,1,2,1,2,1,1,0,1)$, and $a_{4,0}=4, b_{4,0}=112, c_{4,0}=10$. Moreover,

$$
P_{4,0}(t)=1+4 t+31 t^{2}+40 t^{3}+31 t^{4}+4 t^{5}+t^{6} .
$$

In particular, the normalized volume of Gelfand-Tsetlin's polytope $G T_{4,0}$ is equal to

$$
112=2^{4} \cdot 7
$$

It seems interesting to compare the above formulae with the corresponding formulae for the Gelfand-Tsetlin polytope corresponding to the conjugate partition $\lambda^{\prime}=(4,4)$ and the same $\mu$ and $\eta$. It's not difficult to see that $\operatorname{dim} G T((4,4)$, $\left.\left(1^{8}\right)\right)=5, J_{(4,4),\left(1^{8}\right),\left(1^{8}\right)}(q)=\{12,14,15,16,18,20,24\}, P_{(4,4),\left(1^{8}\right),\left(1^{8}\right)}(t)=(1,8$, $22,8,1)$ and $P_{(4,4),\left(1^{8}\right),\left(1^{8}\right)}(q, t)$ is a reciprocal polynomial. In particular, the normalized volume of the polytope $G T\left((4,4),\left(1^{8}\right)\right)$ is equal to 40 .

(ii) Take $k=3, n=2$, then $\operatorname{dim} G T_{3,2}=14, a_{3,2}=10, b_{3,2}=130, c_{3,2}=$ $14, J_{3,2}(q)=q^{3}(1,1,2, \mathbf{3}, 2,2,2,1,1,1,1,1,1)$, but $K_{\left(2^{3}, 1^{2}\right),\left(1^{8}\right)}(q)=q^{3}(1,1,2, \mathbf{2}$, $3,3,4,3,3,2,2,1,1)$. Therefore, the difference $K_{\left(2^{3}, 1^{2}\right),\left(1^{8}\right)}(q)-J_{3,2}(q)$ is a polynomial with one negative coefficient. Moreover,

$$
\begin{aligned}
P_{3,2}(t)= & 1+13 t+225 t^{2}+1350 t^{3} \\
& +4088 t^{4}+5768 t^{5}+4088 t^{6}+1350 t^{7}+225 t^{8}+13 t^{9}+t^{10} .
\end{aligned}
$$

Therefore, the normalized volume of Gelfand-Tsetlin's polytope $G T_{3,2}$ is equal to $17112=2^{3} \cdot 3 \cdot 23 \cdot 31$.

On the other hand, for the conjugate partition $\lambda^{\prime}=(5,3)$ we have $\operatorname{dim} G T$ $\left((5,3),\left(1^{8}\right)\right)=6, J_{(5,3),\left(1^{8}\right),\left(1^{8}\right)}=\{13,14,15,16,17,18,19,22,23,25\}$ and $P_{(5,3),\left(1^{8}\right),\left(1^{8}\right)}(t)=(1,21,105,98,20)$, and therefore, the polynomial $P_{(5,3),\left(1^{8}\right),\left(1^{8}\right)}$ $(q, t)$ does not satisfy the condition (2) of Proposition 5.7.

(iii) Take $k=5, n=0$, then $\operatorname{dim} G T_{5,0}=16$, and

$$
\begin{gathered}
P_{5,0}(t)=(1,25,718,8059,43679,116840,161912,116840, \\
43679,8059,718,25,1) .
\end{gathered}
$$

In particular, the normalized volume of Gelfand-Tsetlin's polytope $G T_{5,0}$ is equal to 
$500556=2^{2} \cdot 3 \cdot 7 \cdot 59 \cdot 101$.

Note that $\operatorname{dim} G T\left((5,5),\left(1^{10}\right)\right)=7, P_{(5,5),\left(1^{10}\right),\left(1^{10}\right)}(t)=(1,34,295,565$, $295,34,1)$, and $J_{(5,5),\left(1^{10}\right),\left(1^{10}\right)}(q)=\{20,22,23,24,25,26,28,30,32,35,40\}$.

In particular, the normalized volume of the polytope $G T\left((5,5),\left(1^{10}\right)\right)$ is equal to $1225=35^{2}$. One can check that $P_{(5,5),\left(1^{10}\right),\left(1^{10}\right)}(q, t)$ is a reciprocal polynomial.

(3) It is interesting to note that the polytopes $G T\left(\left(n^{k}\right),\left(1^{k n}\right)\right)$ and $G T\left(\left(n^{k-1}, n-1\right),\left(1^{k n-1}\right)\right)$ have the same (normalized) volumes and the same $h$-polynomials, i.e.

$$
P_{\left(n^{k}\right),\left(1^{k n}\right),\left(1^{k n}\right)}(t)=P_{\left(n^{k-1}, n-1\right),\left(1^{k n-1}\right),\left(1^{k n-1}\right)}(t) .
$$

However, the polynomials $P_{\left(n^{k}\right),\left(1^{k n}\right),\left(1^{k n}\right)}(q, t)$ and $P_{\left(n^{k-1}, n-1\right),\left(1^{k n-1}\right),\left(1^{k n-1}\right)}$ $(q, t)$ are different.

For example, $P_{(3,3),\left(1^{6}\right),\left(1^{6}\right)}(q, t)=1+q^{10} t+q^{20} t^{2}$, but $P_{(3,2),\left(1^{5}\right),\left(1^{5}\right)}(q, t)=$ $1-q^{20} t^{3}$

Moreover, $J_{(3,3),\left(1^{6}\right),\left(1^{6}\right)}=\{6,8,9,12\}$, but $J_{(3,2),\left(1^{5}\right),\left(1^{5}\right)}=\{4,5,6,7,8\}$.

(\$) It seems an interesting problem to find under what assumptions on $\lambda$, $\mu$ and $\eta$ the polynomial $P_{\lambda \mu \eta}(q, t)$ is a reciprocal one, i.e. satisfies the condition (2) of Proposition 5.7. One necessary condition is clear: $P_{\lambda \mu \eta}(t)$ have to be a symmetric polynomial.

(W) We expect that the latter condition is also sufficient.

For example, the polynomials $P_{\left(2^{k}, 1^{n}\right),\left(1^{2 k+n}\right),\left(1^{2 k+n}\right)}(q, t)$ are reciprocal; we expect that polynomials $P_{\left(n^{k}\right),\left(1^{n k}\right),\left(1^{n k}\right)}(q, t)$ are also reciprocal. However, there are plenty of other cases. For example,

$P_{(4,3,2),(2,1,2,1,2,1),\left(2^{3}\right)}(q, t)=1+q^{5}(-3,2) t-3 q^{11}(1,-1,1) t^{2}+3 q^{16}(1,1$, $1,-2) t^{3}+3 q^{23}(-2,1,1,1) t^{4}-3 q^{29}(1,-1,1) t^{5}-q^{36}(-2,3) t^{6}+q^{42} t^{7}$.

We have also $J_{(4,3,2),(2,1,2,1,2,1),\left(2^{3}\right)}(q)=3 q^{5}(1,1,1)$.

( $)$ On the other hand, we expect that the polynomials $P_{(n, k),\left(1^{n+k}\right),\left(1^{n+k}\right)}$ $(q, t)$ are reciprocal if and only if $k=0,1, n-1, n$.

In the case $k=2$ we can say more:

Proposition 5.12. (1) $\operatorname{deg}_{t} P_{2, n}(q, t)=2 n, \operatorname{deg}_{q} P_{2, n}(q, t)=2 n(n+$ $4)$;

(2) $q^{2 n(n+4)} P_{2, n}\left(q^{-1}, t\right)=P_{2, n}(q, t)$;

(3) $P_{k, n}(q, t)$ is a polynomial with non-negative integer coefficients;

(4) $P_{2, n}(1,1)=C_{n} C_{n+1}$. In other words, the (normalized) volume of the Gelfand-Tsetlin polytope $G T_{2, n}$ is equal to the product of two consecutive 
Catalan numbers $C_{n}$ and $C_{n+1}$;

(5) $J_{2, n}(q)=q^{2}(1,1, \underbrace{2, \ldots, 2}_{n-1}, \underbrace{1, \ldots, 1}_{n+2})$, and

$$
K_{\left(2^{2}, 1^{n}\right),\left(1^{n+4}\right)}(q)-J_{2, n}(q)=q^{6}\left[\begin{array}{l}
n \\
2
\end{array}\right]_{q} .
$$

We end this Section by discussion of some properties of the LittlewoodRichardson coefficients $c_{\delta_{n}, \delta_{n}}^{\lambda}$, where $\delta_{n}=(n-1, n-2, \ldots, 1,0)$ denotes the staircase partition of height $n-1$.

Denote by $\kappa(n, m)$ the maximal value of the $L R$-number $c_{\delta_{n}, \delta_{n}}^{\lambda}$, where $\lambda$ runs over all partitions such that $l(\lambda) \leq m$. Let $v_{n, m}(r)$ denote the number of partitions $\lambda, l(\lambda) \leq m$, such that $c_{\delta_{n}, \delta_{n}}^{\lambda}=r$. It is well-known (theorem by Kostant) that $v_{n, n}(1)=2^{n-1}$.

( We expect that if $n \leq m \leq 2 n-2$, then $v_{n, m}(1)=3^{m-n} / 2^{m-2 n+1}$.

Problem 5.13. It is not difficult to see that $v_{n, n}(\kappa(n, n))=1$, i.e. there exists a unique partition $\lambda:=\lambda_{\max }, l(\lambda) \leq m$, with the maximal value of the Littlewood-Richardson coefficient $c_{\delta_{n}, \delta_{n}}^{\lambda}$.

Question. How does this unique partition $\lambda_{\max }$ look like?

(W) We expect that if $n=2 k+1, k \geq 1$, then

$$
\lambda_{\max }=(3 k+[(k+1) / 2]-1,3 k-1,3 k-2, \ldots, k+1,[k / 2]+1) .
$$

\section{§5.5. One dimensional sums and parabolic Kostka polynomials}

(\$) Polynomials $\mathcal{P}_{\lambda \mu}(q)$ and their interpretations [32]

In this Example we summarize different interpretations and some properties of an interesting family of polynomials $\mathcal{P}_{\lambda \mu}(q)$ which frequently appear in Combinatorics, Algebraic Geometry, Representation Theory, Statistical Mechanics, ....

Definition 5.14. The polynomials $\mathcal{P}_{\lambda \mu}(q)$ are defined as the transition coefficients between the modified Hall-Littlewood polynomials and the monomial symmetric functions

$$
Q_{\lambda}^{\prime}\left(X_{n} ; q\right)=\sum_{\mu} \mathcal{P}_{\lambda \mu}(q) m_{\mu}\left(X_{n}\right) .
$$


In other words,

$$
\mathcal{P}_{\lambda \mu}(q)=\sum_{\eta} K_{\eta \mu}(1) K_{\eta \lambda}(q)
$$

To put this another way, the polynomial $\mathcal{P}_{\lambda \mu}(q)$ is a $q$-analog of the multiplicity of weight $\lambda$ in the tensor product $\otimes_{i} V_{\mu_{i}}$.

The polynomials $\mathcal{P}_{\lambda \mu}(q)$ admit the following interpretations:

$\left(1^{0}\right)$ [Inhomogeneous unrestricted one dimensional sum with "special boundary conditions"]

$$
\mathcal{P}_{\lambda \mu}(q)=q^{n\left(\mu^{\prime}\right)} \sum_{m \in \mathcal{P}_{\lambda \mu}} q^{E(m)},
$$

summed over the set $\mathcal{P}_{\lambda \mu}$ of all transportation matrices $m$ of type $(\lambda ; \mu)$, i.e. the set of all matrices of non-negative integers with row sums $\lambda_{i}$ and column sums $\mu_{j} ; E(m)$ stands for the value of the energy function $E(p)$ of the path $p$ which corresponds to the transportation matrix $m$ under a natural identification, see [32], of the set of paths $\mathcal{P}_{\mu}\left(b_{\max }, \lambda\right)$ with that of transportation matrices $\mathcal{P}_{\lambda \mu}$. We refer the reader to [46], or [32] Subsection 3.1, Example $1^{0}$, for a definition of the set of paths $\mathcal{P}_{\mu}\left(b_{\max }, \lambda\right)$.

$\left(2^{0}\right)$ [Generating function of a generalized mahonian statistics $\varphi$ on the set of transportation matrices $\mathcal{P}_{\lambda \mu}$ ]

$$
\mathcal{P}_{\lambda \mu}(q)=q^{n\left(\mu^{\prime}\right)} \sum_{m \in \mathcal{P}_{\lambda \mu}} q^{\varphi(m)} .
$$

For the definition and examples of generalized mahonian statistics see [32]. For example, the energy function $E(m)$ defines a generalized mahonian statistics on the set of transportation matrices.

$\left(3^{0}\right)$ [The Poincare polynomial of the partial flag variety $\mathcal{F}_{\mu}^{\lambda} / \mathbf{C}$ ]

$$
\mathcal{P}_{\lambda \mu}(q)=\sum_{i \geq 0} q^{n(\lambda)-i} \operatorname{dim} H_{2 i}\left(\mathcal{F}_{\mu}^{\lambda} ; \mathbf{Z}\right) .
$$

This result is due to R. Hotta and N. Shimomura [25].

$\left(4^{0}\right)$ [The number of $\mathbf{F}_{q}-$ rational points of the partial flag variety $\left.\mathcal{F}_{\mu}^{\lambda} / \mathbf{F}_{q}\right]$

$$
q^{n(\lambda)} \mathcal{P}_{\lambda \mu}\left(q^{-1}\right)=\mathcal{F}_{\mu}^{\lambda}\left(\mathbf{F}_{q}\right)
$$


$\left(5^{0}\right)$ [The number of chains of subgroups]

$$
\{e\} \subseteq H^{(1)} \subseteq H^{(2)} \subseteq \cdots \subseteq H^{(m)} \subseteq G
$$

in a finite abelian $p$-group $G$ of type $\lambda$, such that each subgroup $H^{(i)}$ has order $p^{\mu_{1}+\cdots+\mu_{i}}$

$$
\alpha_{\lambda}(S ; p)=p^{n(\lambda)} \mathcal{P}_{\lambda \mu}\left(p^{-1}\right),
$$

where $S:=S(\mu)=\left(\mu_{1}, \mu_{1}+\mu_{2}, \ldots, \mu_{1}+\mu_{2}+\cdots+\mu_{m}\right)$, and $l(\mu)=m+1$. For more details, proofs and an interesting history of this result, see e.g [10].

$\left(6^{0}\right)$ [String function of affine Demazure's module $V_{w}\left(l \Lambda_{L}\right)$ corresponding to the element $w=r_{L n-1} r_{L n-2} \ldots r_{L+2} r_{L+1} r_{L}$ of the affine Weyl group $\left.W\left(A_{n-1}^{(1)}\right)\right]$

$$
\mathcal{P}_{\left(l^{L}\right) \mu}(q) \doteq \sum_{n \geq 0} \operatorname{dim} V_{w}\left(l \Lambda_{L}\right)_{\mu-n \delta} q^{n} .
$$

This result has been obtained in [46], where one can find necessary definitions, proofs and further details.

$\left(7^{0}\right)$ [Generalized $t$-supernomial coefficients $\left[\begin{array}{c}\lambda \\ \mu\end{array}\right]_{t}^{(0)}$ and $t$-multinomial coefficients $\left.T^{(0)}(\lambda ; \mu)\right]$

$$
\begin{aligned}
& {\left[\begin{array}{l}
\lambda \\
\mu
\end{array}\right]_{t}^{(0)}=\sum_{\eta} K_{\eta \mu} \widetilde{K}_{\eta \lambda}(t)=t^{n(\lambda)} \sum_{\eta} K_{\eta \mu} K_{\eta \lambda}\left(t^{-1}\right),} \\
& T^{(0)}(\lambda ; \mu)=t^{-E_{\min }} \mathcal{P}_{\lambda \mu}(t),
\end{aligned}
$$

for some known constant $E_{\text {min }}$.

The coefficients (5.55) and (5.56) are natural generalizations of those introduced by A. Schilling and S.O. Warnaar in the case $l(\mu)=2$, see $[31,62,63,71]$.

$\left(\mathbf{8}^{\mathbf{0}}\right)$ [Fermionic expression for polynomials $\left.\mathcal{P}_{\lambda \mu}(q)\right]$

Let $\lambda$ be a partition and $\mu$ be a composition, $l(\mu)=n$, then

$$
\mathcal{P}_{\lambda \mu}(q)=\sum_{\{\nu\}} q^{c(\{\nu\})} \prod_{k=1}^{n-1} \prod_{i \geq 1}\left[\begin{array}{c}
\left(\nu^{(k+1)}\right)_{i}^{\prime}-\left(\nu^{(k)}\right)_{i+1}^{\prime} \\
\left(\nu^{(k)}\right)_{i}^{\prime}-\left(\nu^{(k)}\right)_{i+1}^{\prime}
\end{array}\right]_{q}
$$

summed over all flags of partitions $\nu=\left\{0=\nu^{(0)} \subset \nu^{(1)} \subset \cdots \subset \nu^{(n)}=\lambda\right\}$, such that $\left|\nu^{(k)}\right|=\mu_{1}+\cdots+\mu_{k}, 1 \leq k \leq n$, and

$$
c(\{\nu\})=\sum_{k=0}^{n-1} \sum_{i \geq 1}\left(\begin{array}{c}
\left(\nu^{(k+1}\right)_{i}^{\prime}-\left(\nu^{(k)}\right)_{i}^{\prime} \\
2
\end{array}\right) .
$$


See [32], Sections 3 and 4, and [22], where further details and applications of the fermionic formula (5.57) can be found.

In particular, the fermionic formula (5.57) gives an explicit expression for the number $\left|\mathcal{F}_{\mu}^{\lambda}\left(\mathbf{F}_{q}\right)\right|$ of rational points of the partial flag variety $\mathcal{F}_{\mu}^{\lambda}$ over the finite field $\mathbf{F}_{q}$.

Problem 5.15. Deduce the fermionic formula (5.57) from the Lefschetz fixed points formula, applied to the Frobenius automorphism of the variety $\mathcal{F}_{\mu}^{\lambda}$.

$\left(9^{0}\right)$ [Truncated form or finitization of the characters and branching functions of (some) integrable representations of the affine Lie algebra of type $A_{n-1}^{(1)}$ ]

The observation that certain special limits of polynomials $\mathcal{P}_{\lambda \mu}(q)$ and Kostka-Foulkes polynomials may play an important role in the representation theory of affine Lie algebras originally was made in [31]. It was observed in [31], that the character formula for the level 1 vacuum representation $V\left(\Lambda_{0}\right)$ of the affine Lie algebra of type $A_{n-1}^{(1)}$ (see, e.g., [26], Chapter 13) can be obtained as an appropriate limit $N \rightarrow \infty$ of the modified Hall-Littlewood polynomials $Q_{\left(1^{N}\right)}^{\prime}\left(X_{n} ; q\right)$. The proof was based on the following well-known formula

$$
\mathcal{P}_{\left(1^{N}\right) \mu}(q)=q^{n\left(\mu^{\prime}\right)}\left[\begin{array}{c}
N \\
\mu_{1}, \ldots, \mu_{n}
\end{array}\right]_{q},
$$

see [31], (2.28).

The latter observation about a connection between the character $\operatorname{ch}\left(V\left(\Lambda_{0}\right)\right)$ and modified Hall-Littlewood polynomials $Q_{\left(1^{N}\right)}^{\prime}\left(X_{n} ; q\right)$, immediately implies that the level 1 branching functions $b_{\lambda}^{\Lambda_{0}}(q)$ can be obtained as an appropriate limit $\lambda_{N} \rightarrow \infty$ of the "normalized" Kostka-Foulkes polynomials $q^{-A_{N}} K_{\lambda_{N},\left(1^{N}\right)}(q)$. We refer the reader to [26], Chapter 12, for definitions and basic properties of the branching functions $b_{\lambda}^{\Lambda}(q)$ corresponding to an integrable representation $V(\Lambda)$ of an affine Lie algebra.

It was conjectured in [31], Conjecture 4, that the similar result should be valid for the branching functions $b_{\lambda}^{\Lambda}(q)$ corresponding to the integrable highest weight $\Lambda$ irreducible representation $V(\Lambda)$ of the affine Lie algebra $\widehat{s l}(n)$. This conjecture has been proved in [31] in the following cases: $\widehat{s l}(n)$ and $\Lambda=\Lambda_{0}$, $\widehat{s l}(2)$ and $\Lambda=l \Lambda_{0}$, and $\widehat{s l}(n)$ and $\Lambda=2 \Lambda_{0}$. It had not been long before A. Nakayashiki and Y. Yamada [58] proved this conjecture in the case $\widehat{s l}(n)$ and $\Lambda=l \Lambda_{i}, 0 \leq i \leq n-1$. See also [39] for another proof of the result by A. Nakayashiki and Y. Yamada in the case $i=0$. The general case has 
been investigated in [22]. It happened that in general the so-called thermodynamical Bethe ansatz limit of Kostka-Foulkes polynomials gives the branching function of a certain reducible integrable representation of $\widehat{s l}(n)$, see details in [22].

(\$) [Parabolic Kostka polynomials and 1D sums]

Let $\lambda, \mu$ be partitions, $|\lambda|=|\mu|$, and $n, N$ be natural numbers such that $l(\lambda)=r \leq n, l(\mu)=s \leq n$, and $N \geq \lambda_{1}+\mu_{1}$. Define partitions $\alpha_{N}=\left(N^{n}\right)$ and

$$
\beta_{N}=\left(N-\lambda_{r}, N-\lambda_{r-1}, \ldots, N-\lambda_{1}, \mu_{1}, \mu_{2}, \ldots, \mu_{s}\right) .
$$

Theorem 5.16 (Algebraic version of the Robinson-SchenstedKnuth correspondence).

Let $\lambda, \mu, n, N, \alpha_{N}$ and $\beta_{N}$ be as above. Then

i) $K_{\alpha_{N} \beta_{N}}(q) \leq K_{\alpha_{N+1} \beta_{N+1}}(q)$;

$$
\text { ii) If } N \geq|\lambda| \text {, then } K_{\alpha_{N} \beta_{N}}(q) \doteq \sum_{\eta} K_{\eta \lambda}(q) K_{\eta \mu}(q) \text {. }
$$

Theorem 5.17 (Algebraic version of the dual Robinson-Schensted-Knuth correspondence).

Let $\lambda, \mu$ be partitions, $|\lambda|=|\mu|, l(\lambda)=r \leq n, N \geq \lambda_{1}$. Define the rectangular shape partition $\alpha_{N}=\left(n^{N}\right)$ and dominant sequence of rectangular shape partitions $R_{N}=\left\{\mu,\left(1^{N-\lambda_{r}}\right), \ldots,\left(1^{N-\lambda_{1}}\right)\right\}$. Then

i) $K_{\alpha_{N} R_{N}}(q) \leq K_{\alpha_{N+1} R_{N+1}}(q)$;

$$
\text { ii) If } N \geq|\lambda| \text {, then } K_{\alpha_{N} R_{N}}(q) \doteq \sum_{\eta} \bar{K}_{\eta \lambda}(q) K_{\eta^{\prime} \mu}(q) \text {. }
$$

In particular, the following numbers

$$
K_{\left(N^{n}\right),\left((n-1)^{\left.N, 1^{N}\right)}\right.}(1)=\sum_{\lambda \vdash N, l(\lambda) \leq n}\left(K_{\lambda,\left(1^{N}\right)}(1)\right)^{2}
$$

are equal to the number of permutations $w \in \Sigma_{N}$ such that the all increasing subsequences in $w$ have the length at most $n$.

Theorem 5.18 (1D sums and parabolic Kostka polynomials).

(i) Let $\lambda$ and $\mu$ be partitions of the same size $n$. Define partition $\alpha_{N}=$ $\left(N^{n}\right)$ and sequence of compositions

$$
\widetilde{\boldsymbol{\mu}}_{N}=\left(\left(N-\lambda_{r}, 0^{r-1}\right),\left(N-\lambda_{r-1}, 0^{r-1}\right), \ldots,\left(N-\lambda_{1}, 0^{r-1}\right), \mu\right) .
$$

Then

$$
K_{\alpha_{N}, \widetilde{\boldsymbol{\mu}}_{N}}(q) \doteq \sum_{\eta} K_{\eta, \lambda}(1) K_{\eta, \mu}(q)=\mathcal{P}_{\mu, \lambda}(q)
$$


(ii) Keep notation of the previous item, but define

$$
\widetilde{\boldsymbol{\mu}}_{N}^{(0)}=\left(N-\lambda_{r}, N-\lambda_{r-1}, \ldots, N-\lambda_{1},\left(\mu_{1}, 0^{(r-1)}\right), \ldots,\left(\mu_{s}, 0^{(r-1)}\right)\right) .
$$

Then

$$
K_{\alpha_{N}, \widetilde{\boldsymbol{\mu}}_{N}^{(0)}}(q) \doteq \sum_{\eta} K_{\eta, \lambda}(q) K_{\eta, \mu}(1)=\mathcal{P}_{\lambda, \mu}(q) .
$$

Example 5.19. Take $n=6, \lambda=(2,2,2)$ and $\mu=(2,2,1,1)$. One can take $N=6$. Then $\alpha_{6}=(6,6,6), \boldsymbol{\mu}_{6}=((4),(4),(4),(2),(2),(1),(1))$, $\widetilde{\boldsymbol{\mu}}_{6}=((4,0,0),(4,0,0),(4,0,0),(2),(2),(1),(1)), \widetilde{\boldsymbol{\mu}}_{6}^{(0)}=((4),(4),(4),(2,0,0)$, $(2,0,0),(1,0,0),(1,0,0))$, and

$$
\begin{aligned}
& \sum_{\eta} K_{\eta, \lambda}(q) K_{\eta, \mu}(q)=q^{7}(1,1,3,3,5,4,6,3,3,2,1,0,1)=K_{\alpha_{6}, \boldsymbol{\mu}}(q), \\
& \sum_{\eta} K_{\eta, \lambda}(q) K_{\eta, \mu}(1)=q^{13}(1,4,8,9,7,3,1)=K_{\alpha_{6}, \widetilde{\boldsymbol{\mu}}_{6}^{(0)}}(q), \\
& \sum_{\eta} K_{\eta, \lambda}(1) K_{\eta, \mu}(q)=q^{31}(3,6,9,7,5,2,1)=K_{\alpha_{6}, \widetilde{\boldsymbol{\mu}}_{6}}(q) .
\end{aligned}
$$

Conjecture 5.20 (Summation formulas for parabolic Kostka polynomials).

(i) Let $\boldsymbol{\mu}=\left(\mu^{(a)}:=\left(\mu_{1}^{(a)}, \ldots, \mu_{\eta_{a}}^{(a)}\right)\right)_{a=1}^{r}$ and $\boldsymbol{\nu}$ be two sequences of partitions such that $|\boldsymbol{\mu}|=|\boldsymbol{\nu}|$. Take $n:=\sum_{a=1}^{r} \eta_{a}$ and $N \geq|\boldsymbol{\mu}|$, and define the sequence of partitions $\widetilde{\boldsymbol{\mu}}:=\left(\widetilde{\mu}^{(r)}, \widetilde{\mu}^{(r-1)}, \ldots, \widetilde{\mu}^{(1)}\right)$, where

$$
\widetilde{\mu}^{(a)}:=\left(N-\mu_{\eta_{a}}^{(a)}, \ldots, N-\mu_{2}^{(a)}, N-\mu_{1}^{(a)}\right) .
$$

Then

$$
K_{\left(N^{n}\right),(\widetilde{\boldsymbol{\mu}}, \boldsymbol{\nu})}(q) \doteq \sum_{\lambda} K_{\lambda, \boldsymbol{\mu}}(q) K_{\lambda, \boldsymbol{\nu}}(q)
$$

(ii) Define the sequence of partitions $\boldsymbol{\mu}_{0}=\left(\mu_{0}^{(a)}:=\left(\mu_{1}^{(a)}, \ldots, \mu_{\eta_{a}}^{(a)}, 0^{\left(N-\eta_{a}\right)}\right)\right)_{a=1}^{r}$ and in a similar way that $\boldsymbol{\nu}_{0}$. Then

$$
\begin{aligned}
& K_{\left(N^{n}\right),\left(\widetilde{\boldsymbol{\mu}}, \boldsymbol{\nu}_{0}\right)}(q) \doteq \sum_{\lambda} K_{\lambda, \boldsymbol{\mu}}(q) K_{\lambda, \boldsymbol{\nu}}(1), \\
& K_{\left(N^{n}\right),\left(\widetilde{\boldsymbol{\mu}}_{0}, \boldsymbol{\nu}\right)}(q) \doteq \sum_{\lambda} K_{\lambda, \boldsymbol{\mu}}(1) K_{\lambda, \boldsymbol{\nu}}(q) .
\end{aligned}
$$

\section{$\S 6 . \quad$ Parabolic Kostka Polynomials: Conjectures}

We keep notation of Section 2. Thus, $\lambda$ is a partition, $\mu$ and $\eta$ are compositions such that $|\lambda|=|\mu|,|\eta|=n$, and $l l(\mu) \leq n$. Let $K_{\lambda \mu \eta}(q)$ denote the parabolic Kostka polynomial as defined in Section 4. 
Definition 6.1. $\quad$ Let $\lambda, \mu$ and $\eta$ be as above, and assume that $K_{\lambda \mu \eta}(q) \neq$ 0 . Introduce non-zero numbers $b(\lambda, \mu \| \eta)$ and $d(\lambda, \mu \| \eta)$, and integer numbers $a(\lambda, \mu \| \eta)$ and $c(\lambda, \mu \| \eta)$ via the decomposition

$$
K_{\lambda \mu \eta}(q)=b(\lambda, \mu \| \eta) q^{a(\lambda, \mu \| \eta)}+\cdots+d(\lambda, \mu \| \eta) q^{c(\lambda, \mu \| \eta)} .
$$

If $K_{\lambda \mu \eta}(q)=0$, we put by definition, $a(\lambda, \mu \| \eta)=b(\lambda, \mu \| \eta)=c(\lambda, \mu \| \eta)=$ $d(\lambda, \mu \| \eta)=0$.

If a composition $\mu$ is the concatenation of partitions $\mu^{(1)}, \mu^{(2)}, \ldots, \mu^{(r)}$, we will use notation $a(\lambda, \boldsymbol{\mu}):=a(\lambda, \mu \| \eta), b(\lambda, \boldsymbol{\mu}):=b(\lambda, \mu \| \eta)$. If compositions $\mu$ and $\eta$ correspond to a (dominant) sequence of rectangular shape partitions $R$, we will write $a(\lambda, R)$ instead of $a(\lambda, \mu \| \eta), b(\lambda, R)$ instead of $b(\lambda, \mu \| \eta)$, and so on.

\section{§6.1. Non-vanishing conjecture}

Conjecture 6.2. $\quad$ Let $\lambda$ be a partition, $\mu$ and $\eta$ be a composition, $l l(\mu) \leq$ $|\eta|=n$. Then

$K_{\lambda \mu \eta}(q) \neq 0$, if and only if $\lambda-\mu \in Y_{\eta}$.

(\$) Moreover, $K_{\lambda \mu \eta}(q) \leq K_{\Phi(\eta)}(\lambda-\mu \mid q)$,

and the equality is attained on a certain polyhedral domain $\mathcal{D}_{\eta}$ in "the space of parameters" $Z_{\eta}=\left\{(\lambda, \mu) \in \mathbb{Z}_{\geq 0}^{n} \times \mathbb{Z}_{\geq 0}^{n} \mid \lambda_{1} \geq \cdots \geq \lambda_{n}, \lambda-\mu \in Y_{\eta}\right\}$.

\section{$\S 6.2$. Positivity conjecture}

Conjecture 6.3. Let $\lambda$ be a partition and $\mu$, and $\eta$ be compositions such that $|\lambda|=|\mu|, l l(\mu) \leq|\eta|$. Then

$$
d(\lambda, \mu \| \eta) \geq 0
$$

Remark 6.4. It may happen that the all coefficients of a parabolic Kostka polynomial $K_{\lambda, \boldsymbol{\mu}}(q)$, except that $d(\lambda, \boldsymbol{\mu})$, are negative. For example, take $\lambda=(2,2)$ and $\boldsymbol{\mu}=((0),(1,0),(1,0),(1),(1))$. Then

$$
K_{n \lambda, n \boldsymbol{\mu}}(q)=-q^{7 n-1}\left[n+\sum_{k=1}^{n}(2 n-2 k+1) q^{k}\right]+(n+1)^{2} q^{8 n} .
$$

Note, that in our example $b(n \lambda, n \boldsymbol{\mu})=-n, a(n \lambda, n \boldsymbol{\mu})=7 n-1, c(n \lambda, n \boldsymbol{\mu})=8 n$, $d(n \lambda, n \boldsymbol{\mu})=(n+1)^{2}, K_{n \lambda, n \boldsymbol{\mu}}(1)=n+1, K_{n \lambda, n \boldsymbol{\mu}}(-1)=(n+1)^{2}$, and $\sum_{n \geq 0} K_{n \lambda, n \boldsymbol{\mu}}(q) t^{n}=\left(1-q^{6}\left(1+3 q-q^{2}\right) t+3 q^{14} t^{2}-q^{23} t^{3}\right) /\left(1-q^{7} t\right)^{2}\left(1-q^{8} t\right)^{3}$. 
On the other hand,

$$
K_{(2 n, 2 n),(n, n, n, n)}(q)=q^{2 n}\left[\begin{array}{c}
n+1 \\
1
\end{array}\right]_{q^{2}} .
$$

\section{$\S 6.3$. Generalized saturation conjecture for parabolic Kostka polynomials}

\section{Conjecture 6.5 $($ ) (Generalized Saturation Conjecture).}

Let $\lambda$ be a partition, and $\mu$ and $\eta$ be compositions, then for any integer $N \geq 1$

$$
c(N \lambda, N \mu \| \eta)=N c(\lambda, \mu \| \eta)
$$

$(\downarrow)$ Let $\lambda$ and $\mu$ be partitions and $\eta$ be a composition, then for any integer $N \geq 1$

$$
a(N \lambda, N \mu \| \eta)=N a(\lambda, \mu \| \eta)
$$

$(\diamond \diamond)$ More generally, let $\lambda^{(1)}, \lambda^{(2)}, \ldots, \lambda^{(s)}$ be a sequence of partitions, $\eta$ be a composition and $\mu^{(1)}, \mu^{(2)}, \ldots, \mu^{(s)}$ be a sequence of compositions such that $\left|\lambda^{(j)}\right|=\left|\mu^{(j)}\right|$ and $l l\left(\mu^{(j)}\right) \leq|\eta|$ for all $j$. Let $N, p_{1}, p_{2}, \ldots, p_{s}$ be positive integer numbers.

For each $i, 1 \leq i \leq N$, define partitions

$$
\begin{aligned}
\widehat{\lambda}^{(i)} & :=\left[\left(\sum_{j \geq 1}^{s} p_{j} \lambda^{(j)}+N-i\right) / N\right] \text { and } \widehat{\mu}^{(i)} \\
& :=\left[\left(\sum_{j \geq 1}^{s} p_{j} \mu^{(j)}+N-i\right) / N\right] .
\end{aligned}
$$

Assume that $\left|\widehat{\lambda}^{(j)}\right|=\left|\widehat{\mu}^{(j)}\right|$ for all $j$. Then

$$
\sum_{j=1}^{s} p_{j} c\left(\lambda^{(j)}, \mu^{(j)} \| \eta\right)=\sum_{i=1}^{N} c\left(\widehat{\lambda}^{(i)}, \widehat{\mu}^{(i)} \| \eta\right) .
$$

( If $\lambda$ and $\mu$ are partitions, then we expect the similar conjecture for the numbers $a(\lambda, \mu \| \eta)$.

Remember that for any real number $x$ the symbol $[x]$ denotes the integer part of $x$. 
Remark 6.6. It is not true in general that the inequality

$$
\operatorname{deg} K_{\Phi(\eta)}(w(\lambda+\delta)-\mu-\delta \mid q)<\operatorname{deg} K_{\Phi(\eta)}(\lambda-\mu \mid q)
$$

holds for any permutation $w \in \Sigma_{n}, w \neq i d$, as it happens in the case $\eta=\left(1^{n}\right)$, see Example 4.2. If it would be so, the Generalized Saturation Conjecture would follow easily from Saturation Theorem for the parabolic $q$-Kostant partition function, see Corollary 3.14. It is also not true in general that

$$
c(\lambda, \mu \| \eta):=\operatorname{deg} K_{\lambda \mu \eta}(q)=\operatorname{deg} K_{\Phi(\eta)}(\lambda-\mu \mid q),
$$

even if $\mu$ is a dominant sequence of rectangular shape partitions of the same length which is compatible with $\eta$, see Example 4.2. In fact, it looks a difficult problem to find an explicit formula for the numbers $c(\lambda, \mu \| \eta)$.

( However, we expect the validity of the following inequality

$$
K_{\lambda \mu \eta}(q) \leq K_{\Phi(\eta)}(\lambda-\mu \mid q)
$$

and if $\lambda$ is a partition and $R=\left(R_{a}:=\left(\mu_{a}^{k}\right)_{a=1}^{p}\right)$ is a dominant sequence of rectangular shape partitions of the same length $k$, then $d(\lambda, R)=1$.

( By duality, we expect that if $R=\left(R_{a}:=\left(k^{\eta_{a}}\right)_{a=1}^{p}\right)$ is a sequence of rectangular shape partitions of the same width $k$, then $b(\lambda, R)=1$.

\section{$\S 6.4$. Rationality conjecture}

Conjecture 6.7. Let $\lambda$ be a partition, and $\mu$ and $\eta$ be compositions such that $\lambda-\mu \in Y_{\eta}$, and (according to Theorem 4.14)

- $\sum_{n \geq 0} K_{n \lambda, n \mu, \eta}(q) t^{n}=P_{\lambda \mu \eta}(q, t) / Q_{\lambda \mu \eta}(q, t)$,

where $P_{\lambda \mu \eta}(q, t)$ and $Q_{\lambda \mu \eta}(q, t)$ are mutually prime polynomials with integer coefficients, $P_{\lambda \mu \eta}(0,0)=1$,

- $Q_{\lambda \mu \eta}(q, t)=\prod_{j \in J}\left(1-q^{j} t\right)^{n_{j}}$

for some finite set of integers $J:=J_{\lambda \mu \eta}=\left\{j_{\min }=j_{1}<j_{2}<\cdots<j_{s}=j_{\max }\right\}$, and a set of non-negative integers $\left\{n_{j}\right\}_{j \in J}$.

(४) Let $P_{\lambda \mu \eta}(q, t)=\sum_{k \geq 0} P_{\lambda \mu \eta}^{(k)}(q) t^{k}, P_{\lambda \mu \eta}^{(0)}(q)=1$, and $\left(\right.$ if $\left.P_{\lambda \mu \eta}^{(k)}(q) \neq 0\right)$

$$
P_{\lambda \mu \eta}^{(k)}(q)=\beta_{k}(\lambda \mu \eta) q^{\alpha_{k}(\lambda \mu \eta)}+\cdots+\delta_{k}(\lambda \mu \eta) q^{\gamma_{k}(\lambda \mu \eta)} .
$$

Then, for all $k>0$ such that $P_{\lambda \mu \eta}^{(k)}(q) \neq 0$, the following inequalities

$$
\gamma_{k}(\lambda \mu \eta) \leq k j_{\max }
$$


have to be valid. Moreover, if the equality is attained for some value of $k$, then for the corresponding value of $k$ one should have $\delta_{k}(\lambda \mu \eta) \geq 0$.

$(\diamond)$ If $\lambda$ and $\mu$ are partitions, then additionally, for all $k>0$ such that $P_{\lambda \mu \eta}^{(k)}(q) \neq 0$, the following inequalities

$$
\alpha_{k}(\lambda \mu \eta) \geq k j_{\min }
$$

have to be valid, and if the equality is attained for some $k$, then for the corresponding value of $k$ one should have $\beta_{k}(\lambda \mu \eta) \geq 0$.

It follows from Remark 4.22, (\$), that the polynomial $P_{\lambda \mu \eta}^{(1)}(q)$ may have negative integer coefficients.

It is easily seen that Rationality Conjecture, item (i), implies both Positivity and Generalized Saturation Conjectures. Rationality Conjecture, item, (ii), implies the item (ii) of Conjecture 6.5.

Question 6.8. $\quad$ Does there exist a "nice" combinatorial interpretation of the set $J:=J_{\lambda \mu \eta}$ and the exponents $\left\{n_{j}\right\}_{j \in J}$ which have appeared in Rationality Conjecture?

Examples 6.9. For the reader's convenience, we list below a few examples of the set $J_{\lambda \mu \eta}$.

(i) $J_{(3,3,2,1),(2,1,2,1,2,1),\left(2^{3}\right)}=\left\{3^{2}, 4^{3}, 5^{3}, 6^{2}\right\}, \operatorname{deg}_{t} P_{\lambda \mu \eta}(q, t)=8$.

(ii) $J_{(4,2,2,1),(2,1,2,1,2,1),\left(2^{3}\right)}=\left\{4^{4}, 5^{6}, 6^{3}\right\}, \operatorname{deg}_{t} P_{\lambda \mu \eta}(q, t)=10$.

(iii) $J_{(5,4,2,1),\left(3,2^{4}, 1\right),\left(2^{3}\right)}=\left\{4,5^{2}, 6^{3}, 7^{2}\right\}$, $\operatorname{deg}_{t} P_{\lambda \mu \eta}(q, t)=5$.

(iv) $J_{(5,4,2,1),\left(3,2^{4}, 1\right),\left(2,1^{2}, 2\right)}=\left\{4,5^{2}, 6^{3}, 7^{2}, 8^{2}, 9^{2}\right\}, \operatorname{deg}_{t} P_{\lambda \mu \eta}(q, t)=9$.

(v) $J_{(2,2),\left(0^{4}, 1,3\right),\left(1^{6}\right)}=\left\{5,6,7,8,9^{2}, 10,11,12,13,15,17\right\}, \operatorname{deg}_{t} P_{\lambda \mu \eta}(q, t)$ $=12$.

(vi) $J_{(4,4,2,2),\left(2^{6}\right),\left(1^{6}\right)}=\left\{4,6,8^{3}, 10,12,14,16\right\}, \operatorname{deg}_{t} P_{\lambda \mu \eta}(q, t)=7$ and $P_{(4,4,2,2),\left(2^{6}\right),\left(1^{6}\right)}(q, t)$ is a reciprocal polynomial.

(vii) $J_{(4,3,2,1),\left(2^{5}\right),\left(1^{5}\right)}=\{3,4,5,6,7,8,9,10\}, \operatorname{deg}_{t} P_{\lambda \mu \eta}(q, t)=6$ and $J_{(4,3,2,1),\left(2^{5}\right),\left(1^{5}\right)}(q, t)$ is a reciprocal polynomial.

(viii) $J_{(4,3,2,1),\left(1^{10}\right),\left(2^{5}\right)}=\left\{10,11^{3}, 12^{3}, 13^{2}, 14^{2}, 15^{2}, 16,17\right\}, \operatorname{deg}_{t} P_{\lambda \mu \eta}(q, t)$ $=8$, but $P_{(4,3,2,1),\left(1^{10}\right),\left(2^{5}\right)}(q, t)$ is not a reciprocal polynomial. $45,50\}$,

(ix) $J_{(6,5),\left(1^{11}\right),\left(1^{11}\right)}=\{25,26,27,28,29,30,31,33,34,35,37,40,41,43$, $\operatorname{deg}_{t} P_{\lambda \mu \eta}(q, t)=16$ and $P_{(6,5),\left(1^{11}\right),\left(1^{11}\right)}(q, t)$ is a reciprocal polynomial.

(x) $J_{\left(2^{4}, 1\right),\left(1^{9}\right),\left(1^{9}\right)}(q)=q^{4}(1,1,1,2,3,2,2,2,3,2,2,2,1,1,1,1,1), K_{\left(2^{4}, 1\right),\left(1^{9}\right)}$ $(q)-J_{\left(2^{4}, 1\right),\left(1^{9}\right),\left(1^{9}\right)}(q)=q^{9}(1,2,2,1,2,2,1,2,1), \operatorname{deg}_{t} P_{\lambda \mu \eta}(q, t)=23$, and $P_{\left(2^{4}, 1\right),\left(1^{9}\right),\left(1^{9}\right)}(q, t)$ is a reciprocal polynomial. 
(W) We expect that if $\eta_{1}$ and $\eta_{2}$ are two compositions such that $\eta_{2}$ is a subdivision of $\eta_{1}$, then $J_{\lambda, \mu, \eta_{1}} \subseteq J_{\lambda, \mu, \eta_{2}}$.

\section{§6.5. Polynomiality conjecture}

Conjecture 6.10. ( $\quad$ Let $\lambda$ be a partition, $\mu$ and $\eta$ be compositions. Then $d(N \lambda, N \mu \| \eta)$ is a polynomial in $\mathrm{N}$ with non-negative rational coefficients of the following form:

there exist a non-negative integer $D$ and a sequence of non-negative integers

$h_{0}=1, h_{1}, \ldots, h_{D}(\neq 0)$ such that

$$
d(N \lambda, N \mu \| \eta)=\sum_{k=0}^{D} h_{k}\left(\begin{array}{c}
N+D-k \\
D
\end{array}\right) .
$$

$(\diamond)$ Let $\lambda$ be a partition, $\mu$ and $\eta$ be compositions, and

$$
q^{c(n \lambda, n \mu \| \eta)} K_{n \lambda, n \mu, \eta}\left(q^{-1}\right)=\sum_{k \geq 0} d_{\lambda \mu \eta}(k ; n) q^{k},
$$

so that $d_{\lambda \mu \eta}(0 ; N)=d(N \lambda, N \mu \| \eta)$.

Then for a fixed $k \geq 0$, there exists a polynomial with rational coefficients $D_{\lambda \mu \eta}^{(k)}(t)$ of degree depending only on $\lambda, \mu$ and $\eta$, but not $k$, such that if $N \geq k$, then $d_{\lambda \mu \eta}(k ; N)=D_{\lambda \mu \eta}^{(k)}(N)$.

Hence, there exists the limit

$$
\lim _{n \rightarrow \infty} q^{c(n \lambda, n \mu \| \eta)} K_{n \lambda, n \mu, \eta}\left(q^{-1}\right) / d(n \lambda, n \mu \| \eta) .
$$

( $)$ Moreover, we expect that $D_{\lambda \mu \eta}^{(0)}(t)$ and $D_{\lambda \mu \eta}^{(1)}(t)$ have non-negative coefficients.

$(\diamond \diamond)$ Let $\lambda$ and $\mu$ be partitions, and

$$
K_{n \lambda, n \mu, \eta}(q)=q^{a(n \lambda, n \mu \| \eta)}\left\{\sum_{k \geq 0} b_{\lambda \mu \eta}(k ; n) q^{k}\right\},
$$

so that $b_{\lambda \mu \eta}(0 ; N)=b(N \lambda, N \mu \| \eta)$.

Then for a fixed $k \geq 0$, there exists a polynomial with rational coefficients $B_{\lambda \mu \eta}^{(k)}(t)$ of degree depending only on $\lambda, \mu$ and $\eta$, but not $k$, such that if $N \geq k$, then $b_{\lambda \mu \eta}(k ; N)=B_{\lambda \mu \eta}^{(k)}(N)$.

Hence, there exists the limit

$$
\lim _{n \rightarrow \infty} q^{-a(n \lambda, n \mu \| \eta)} K_{n \lambda, n \mu, \eta}(q) / b(n \lambda, n \mu \| \eta) .
$$


(W) Moreover, we expect that $B_{\lambda \mu \eta}^{(0)}(t)$ and $B_{\lambda \mu \eta}^{(1)}(t)$ have non-negative coefficients.

$(\triangleleft \diamond \triangleleft)$ Let $\left\{\left(\lambda_{a}, \mu^{(a)}\right)\right\}_{a=1}^{(r)}$ be a collection of pairs $\left(\lambda_{a}, \mu^{(a)}\right)$, where for all $a, 1 \leq a \leq r, \lambda_{a}$ is a partition and $\mu^{(a)}$ is a composition of the fake length at most $n$. Let $\eta$ be a composition of size $n$. Then, there exists a piecewise polynomial function $\mathcal{M}\left(t_{1}, \ldots, t_{r}\right)$ with rational coefficients such that for each $r$-tuples of non-negative integers $\left(n_{1}, \ldots, n_{r}\right)$ one has

$$
\mathcal{M}\left(n_{1}, \ldots, n_{r}\right)=d\left(n_{1} \lambda_{1}+\cdots+n_{r} \lambda_{r}, n_{1} \mu_{1}+\cdots+n_{r} \mu_{r} \| \eta\right) .
$$

(W) Moreover, we expect that if all compositions $\mu^{(a)}$ 's are in fact partitions, then the restriction of $\mathcal{M}\left(t_{1}, \ldots, t_{r}\right)$ on "the dominant chamber" $\left\{\left(n_{1} \geq \cdots \geq\right.\right.$ $\left.\left.n_{r}\right) \in \mathbb{Z}_{\geq 0}^{r}\right\}$ is a polynomial wit non-negative rational coefficients.

Let us note that Polynomiality Conjecture, items $(\bullet)-(\downarrow \downarrow)$, follow from Rationality Conjecture, except the statements about non-negativity.

Example 6.11. Take $\lambda=(5,3,3,2), \mu=(3,3,3,2,1,1)$ and $\eta=\left(1^{6}\right)$. Based on formulas from Example 4.17 (i), one can find that

$$
\lim _{n \rightarrow \infty} q^{-a(n \lambda, n \mu \| \eta)} K_{n \lambda, n \mu, \eta}(q) / b(n \lambda, n \mu \| \eta)=(1-q)^{-6}(1+q)^{-2} .
$$

Remark 6.12. Even in the case when $\boldsymbol{\mu}$ is a dominant sequence of rectangular shape partitions, the sequence $\left(h_{0}, h_{1}, \ldots, h_{D}\right)$ does not necessarily turn out to be unimodal. For example, take $\lambda=(5,4,3,2,1), \mu=(2,2,2)$ and $\nu=(6,5,4,3,2,1)$. It is not difficult to compute the corresponding LittlewoodRichardson numbers:

$$
\begin{aligned}
c_{n \lambda, n \mu}^{n \nu} & =K_{\left(n(2,2,2), n\left(1^{6}\right)\right)}(1)=\left(\begin{array}{c}
n+4 \\
4
\end{array}\right)+\left(\begin{array}{c}
n+2 \\
4
\end{array}\right) \\
& =(n+1)(n+2)\left(n^{2}+3 n+6\right) / 12 .
\end{aligned}
$$

Hence, in this case $D=2$ and $\left(h_{0}, h_{1}, h_{2}\right)=(1,0,1)$. It is not difficult to check that

$\sum_{n \geq 0} K_{n(2,2,2), n\left(1^{6}\right)}(q) t^{n}=\left(1+q^{15} t^{2}\right) /\left(1-q^{3} t\right)\left(1-q^{5} t\right)\left(1-q^{6} t\right)\left(1-q^{7} t\right)\left(1-q^{9} t\right)$.

We see that in our example $P_{\lambda \mu \eta}^{(1)}(q)=0$. We can show that $P_{\left(2^{4}\right),\left(1^{8}\right),\left(1^{8}\right)}^{(1)}(q)=0$ as well.

(W) However, we expect that if $n \geq 5$, then $P_{\left(2^{n}\right),\left(1^{2 n}\right),\left(1^{2 n}\right)}^{(1)}(q) \neq 0$.

For example, $P_{\left(2^{5}\right),\left(1^{10}\right),\left(1^{10}\right)}^{(1)}(q)=q^{10}(-1,1,1,2,2,2,2,3,1,2,1)$. 
Let us observe that $c_{(5,4,3,2,1),(2,2,2)}^{(6,5,4,2,2)}=5$ is equal to the third Catalan number $C_{3}$. More generally, one can show that

$$
c_{(2 n-1,2 n-2, \ldots, 2,1),\left(2^{n}\right)}^{(2 n, 2 n-1, \ldots, 2,1)}=K_{\left(2^{n}\right),\left(1^{2 n}\right)}(1)=\frac{1}{n+1}\left(\begin{array}{c}
2 n \\
n
\end{array}\right)=C_{n}
$$

is equal to the $n$-th Catalan number.

For definition of unimodal sequences/polynomials see e.g. [66], where one can find a big variety of examples of unimodal sequences which frequently appear in Algebra, Combinatorics and Geometry.

Remark 6.13. In the particular case when $b(\Lambda, R)=c_{\lambda, \mu}^{\nu}$, see Section 5.2 , the fact that the function $f_{\lambda, \mu}^{\nu}(N):=c_{N \lambda, N \mu}^{N \nu}$ is a polynomial in $N$ with rational coefficients follows from Polynomiality Theorem for parabolic Kostka polynomials, see Corollary 4.15, and has been proved independently by the several authors: A. Knutson (unpublished), H. Derksen and J. Weyman [15], E. Rassart [61], ... .

We would like to state separately two particular cases of Conjecture 6.10.

§6.6. The generalized Fulton, $d(\lambda, \mu \| \eta)=2$ and $d(\lambda, \mu \| \eta)=3$

\section{conjectures}

\section{Conjecture 6.14 $($ ) (The generalized Fulton conjecture).}

If $d(k \lambda, k \mu \| \eta)=1$ for some positive integer $k$, then $d(N \lambda, N \mu \| \eta)=1$ for all positive integers $N$.

$(\diamond)$ If $d(\lambda, \mu \| \eta)=2$, then $d(N \lambda, N \mu \| \eta)=N+1$ for all positive integers $N$.

If $d(\lambda, \mu \| \eta)=3$, we expect that there are only two possibilities:

either $d(N \lambda, N \mu \| \eta)=2 N+1$, or $d(N \lambda, N \mu \| \eta)=\left(\begin{array}{c}N+2 \\ 2\end{array}\right)$.

(W) Therefore, we expect that the cases $d(N \lambda, N \mu \| \eta)=\left(\begin{array}{c}N+2 \\ 2\end{array}\right)$ $+k\left(\begin{array}{c}N \\ 2\end{array}\right)$

$1 \leq k \leq 3$, do not occur. For example, we don't know whether or not there exist a partition $\lambda$ and a dominant sequence of rectangular shape partitions $R$ such that $d(\lambda, R)=3$, but $d(2 \lambda, 2 R) \geq 7$. 
Remark 6.15. In the case when the numbers $b(\lambda, R)$ coincide with the Littlewood-Richardson numbers, see Section 5.2, the Fulton conjecture has been proved by A. Knutson, T. Tao and C. Woodward [44].

Remark 6.16. If $\mu$ is a composition, but not a partition, then Conjecture $6.14(\checkmark)$ is not, in general, valid for the numbers $b(\lambda, \mu \| \eta)$. For example, take $\lambda=(3,2,1)$ and $\boldsymbol{\mu}=((0),(2,0),(2),(2))$, see Examples 4.6. Then $a(\lambda, \boldsymbol{\mu})=3, b(\lambda, \boldsymbol{\mu})=1$, but $a(2 \lambda, 2 \boldsymbol{\mu})=7, b(2 \lambda, 2 \boldsymbol{\mu})=3$ and $a(3 \lambda, 3 \boldsymbol{\mu})=$ $8, b(3 \lambda, 3 \boldsymbol{\mu})=-1$. In fact, if $n \geq 3$, then $a(n \lambda, n \boldsymbol{\mu})=3 n-1, b(n \lambda, n \boldsymbol{\mu})=2-n$. On the other hand, $c(n \lambda, n \boldsymbol{\mu})=9 n$ and $d(n \lambda, n \boldsymbol{\mu})=n+1, \forall n \geq 1$. In particular, we see that $b(N \lambda, N \boldsymbol{\mu})$ becomes a polynomial in $N$ only starting from $N=3$.

\section{$\S 6.7 . \quad q$-Log concavity and $P$-positivity conjectures}

\section{Conjecture 6.17 ( $q$-Log concavity and $P$-positivity conjectures).} als)

$(\checkmark)$ ( $q$ Log concavity conjecture for parabolic Kostka polynomi-

(a) Let $\lambda$ and $\mu$ be partitions and $\eta$ be a composition. Consider the function $g_{N}(q):=g_{N}^{\lambda \mu \eta}(q)=K_{N \lambda, N \mu, \eta}(q)$. Then

$$
\left(g_{N}(q)\right)^{2} \geq g_{N-1}(q) g_{N+1}(q) .
$$

(W) Moreover, we expect that if a composition $\eta_{2}$ is a subdivision of that $\eta_{1}$, then

$$
\left(g_{N}^{\lambda \mu \eta_{2}}(q)\right)^{2}-g_{N-1}^{\lambda \mu \eta_{2}}(q) g_{N+1}^{\lambda \mu \eta_{2}}(q) \geq\left(g_{N}^{\lambda \mu \eta_{1}}(q)\right)^{2}-g_{N-1}^{\lambda \mu \eta_{1}}(q) g_{N+1}^{\lambda \mu \eta_{1}}(q) \geq 0 .
$$

(b) More generally, let $\lambda^{(1)}, \lambda^{(2)}, \ldots, \lambda^{(s)}$ and $\mu^{(1)}, \ldots, \mu^{(s)}$ be two sequences of partitions, and $\eta$ be a composition such that $\left|\lambda^{(j)}\right|=\left|\mu^{(j)}\right|$ and $l l\left(\mu^{(j)}\right) \leq|\eta|, 1 \leq j \leq s$. Let $N, p_{1}, p_{2}, \ldots, p_{s}$ be positive integer numbers. Assume that $\left|\widehat{\lambda}^{(j)}\right|=\left|\widehat{\mu}^{(j)}\right|$ for all $j$. Then

$$
\prod_{j=1}^{s}\left(K_{\lambda^{(j)}, \mu^{(j)}, \eta}(q)\right)^{p_{j}} \leq \prod_{i=1}^{N} K_{\widehat{\lambda}^{(i)}, \widehat{\mu}^{(i)}, \eta}(q) .
$$

See Conjecture $6.5,(\triangleleft \triangleleft),(6.62)$, for the explanation of notation $\widehat{\lambda}^{(i)}$ and $\widehat{\mu}^{(i)}$.

( In particular, we expect that if $\lambda:=\left(\lambda^{(1)}+\cdots+\lambda^{(s)}\right) / N$ and $\mu:=$ $\left(\mu^{(1)}+\cdots+\mu^{(s)}\right) / N$ are partitions, then

$$
\prod_{j=1}^{s} K_{\lambda^{(j)}, \mu^{(j)}, \eta}(q) \leq\left(K_{\lambda \mu \eta}(q)\right)^{N} .
$$


(c) (Strong $q$-log concavity conjecture for parabolic Kostka polynomials)

Let $l \geq k \geq r \geq 1$ be integers, $\lambda, \mu$ and $\eta$ be as in Conjecture $6.17(a)$, $g_{n}(q)=K_{n \lambda, n \mu, \eta}(q)$. Then

$$
g_{k}(q) g_{l}(q) \geq g_{k-r}(q) g_{l+r}(q)
$$

( M) Moreover, we expect that the difference $g_{k}(q) g_{l}(q)-g_{k-r}(q) g_{l+r}(q)$ is a unimodal polynomial.

$(\downarrow)(P$-positivity conjecture for parabolic Kostka numbers)

Let $\alpha \supset \beta$ be partitions, $l(\alpha)=r$. Consider the following polynomial:

$$
g_{\alpha \backslash \beta}(q):=g_{\alpha \backslash \beta}^{\lambda \mu \eta}(q)=\operatorname{det}\left(g_{\alpha_{i}-\beta_{j}-i+j}(q)\right)_{1 \leq i, j \leq r} .
$$

Then $g_{\alpha \backslash \beta}(1) \geq 0$. Equivalently, $\left\{g_{N}^{\lambda \mu \eta}(1)\right\}_{N \geq 1}$ is a Pólya frequency sequence.

Remark 6.18. If $r \geq 3$, then it's not true, in general, that all the coefficients of polynomial $g_{\alpha \backslash \beta}(q)$ are non-negative. For example, take $\lambda=$ $(4,3,2,1), R=((2,2),(2),(2),(1,1))$ and $\alpha=(2,2,2)$. Then $g_{\alpha}(q)=4 q^{22}+$ $28 q^{23}+\cdots+7 q^{34}-q^{35}$.

We want to state some special cases of Conjecture 6.17 in its own right.

Conjecture 6.19 $($ ) (The generalized Okounkov conjecture, I).

Let $\lambda$ be a partition and $R$ be a dominant sequence of rectangular shape partitions. Then

$$
(b(N \lambda, N R))^{2} \geq b((N-1) \lambda,(N-1) R) b((N+1) \lambda,(N+1) R) .
$$

$(\diamond)$ More generally, let $\lambda$ and $\mu$ be partitions, and $\eta$ be a composition, then the power series

$$
B(t)=\sum_{n \geq 0} b(n \lambda, n \mu \| \eta) t^{n}
$$

is a $P$-series.

Remind that a power series $B(t)=\sum_{n \geq 0} b_{n} t^{n}$ is called a $P$-series, if $\operatorname{det}\left(b_{\lambda_{i}-i+j}\right) \geq 0$ for any partition $\lambda$.

$(\diamond \diamond)$ Let $\lambda, \mu, \nu$ be partitions, then

$$
c_{[(\lambda+\mu+1) / 2],[(\lambda+\mu) / 2]}^{\nu} \geq c_{\lambda, \mu}^{\nu} .
$$

For a more general conjecture, see Section 6.8. 
In the case then $(\lambda+\mu) / 2$ is a partition, Conjecture 6.19 , $(\diamond \diamond)$, was stated by A. Okounkov [60], Section 2.5. More generally,

( $)$ we expect that for a sequence of partitions $\lambda^{(1)}, \ldots, \lambda^{(p)}$, the difference of products of Schur functions

$$
\prod_{k=1}^{p} s_{\left[\left(\sum_{j} \lambda^{(j)}+p-k\right) / p\right]}-\prod_{j=1}^{p} s_{\lambda^{(j)}}
$$

is a Schur or s-positive, i.e. the latter difference can be written as a linear combination of Schur functions with non-negative (integer) coefficients, cf Conjecture $6.23(\diamond)$.

In the case of the Littlewood-Richardson numbers Conjecture 6.18, ( ), was stated by A. Okounkov [60].

Remark 6.20. The log-concavity of numbers

$$
\operatorname{dim} V_{\lambda}^{\mathfrak{g} l(n)}=s_{\lambda}(\underbrace{1, \ldots, 1}_{n})=\left(\begin{array}{c}
n \\
\lambda^{\prime}
\end{array}\right),
$$

which can be in a natural way identified with certain numbers $b(\lambda, R)$ for some partitions $\lambda$ and dominant sequences of rectangular shape partitions $R$, see e.g. [30], has been proved by A. Okounkov [59].

The $q$-log-concavity of the generalized $q$-Gaussian coefficients for general partition $\lambda$ has been proved by A. Okounkov [59], and earlier for some special cases, by L. Butler, C. Krattenthaller, B. Sagan and others. In fact, A. Okounkov has proved more fine result, namely, that not only the dimension of an irreducible representation (or its $q$-dimension), but the whole skew Schur function is log-concave.

( We expect, that the modified parabolic skew Hall-Littlewood function is $q$-log-concave as well.

\section{$\S 6.8$. The generalized Fomin-Fulton-Li-Poon conjectures}

Let $A=\Lambda \backslash \lambda$ and $B=M \backslash \mu$ be skew diagrams and $\nu$ be a partition. Let $\theta$ be a composition such that $l(\nu) \leq|\theta|$. Define partitions

$$
\alpha=\alpha(A, B):=\left(\left(M_{1}^{\Lambda_{1}^{\prime}}\right)+\Lambda, M\right), \eta=\left(\Lambda_{1}^{\prime}+M_{1}^{\prime}, \theta\right)
$$

and the composition $\beta=\beta(A, B):=\left(\left(M_{1}^{\Lambda_{1}^{\prime}}\right)+\lambda, \mu, 0^{M_{1}^{\prime}-\mu_{1}^{\prime}}, \nu\right)$. 
One can prove that the ratio

$$
K_{A, B, \theta}^{\nu}(q):=q^{-|\nu|} K_{\alpha \beta \eta}(q)
$$

is in fact a polynomial in $q$ with non-negative integer coefficients.

More generally, cf Section 5.2, let $A^{(1)}=\Lambda^{(1)} \backslash \lambda^{(1)}, \ldots, A^{(k)}=\Lambda^{(k)} \backslash \lambda^{(k)}$ be a $k$-tuples of skew diagrams, $\nu$ and $\theta$ be compositions such that $l l(\nu) \leq|\theta|$. Define new partitions $\alpha=\alpha\left(A^{(1)}, \ldots, A^{(k)}\right)$ and $\beta=\beta\left(A^{(1)}, \ldots, A^{(k)}\right)$ in the following way:

$$
\begin{aligned}
& \text { if } \sum_{i \leq r-1} \Lambda_{1}^{(i)^{\prime}}<j \leq \sum_{i \leq r} \Lambda_{1}^{(i)^{\prime}}, \text { for some } 1 \leq r \leq k, \\
& \text { then } \alpha_{j}=\sum_{i=r+1}^{k} \Lambda_{1}^{(i)}+\Lambda_{j}^{(r)}, \beta_{j}=\sum_{i=r+1}^{k} \Lambda_{1}^{(i)}+\lambda_{j}^{(r)},
\end{aligned}
$$

where we put by definition, $\Lambda^{(0)}=\lambda^{(0)}=\emptyset$. In addition, define $\eta=\left(\sum_{i=1}^{k-1} \Lambda_{1}^{(i)^{\prime}}\right.$ $\left.+\lambda_{1}^{(k)^{\prime}}, \theta\right)$.

One can prove that the ratio

$$
K_{A^{(1)}, \ldots, A^{(k)}, \theta}^{\nu}(q):=q^{-|\nu|} K_{\alpha \beta \eta}(q)
$$

is a polynomial in $q$ with non-negative integer coefficients.

The main intention of this Section is to state a few results, examples and conjectures about the latter polynomials.

Proposition 6.21. If $\theta=\left(1^{|\nu|}\right)$, then

(a) $K_{A^{(1)}, \ldots, A^{(k)}, \theta}^{\nu}(0)=c_{A^{(1)}, \ldots, A^{(k)}}^{\nu}$,

where $c_{A^{(1)}, \ldots, A^{(k)}}^{\nu}:=\left\langle s_{A^{(1)}} \ldots s_{A^{(k)}}, s_{\nu}\right\rangle$. Remember, that $s_{A^{(i)}}$ denotes the skew Schur function corresponding to the skew diagram $A^{(i)}$, and $\langle$,$\rangle denotes$ the scalar product (the so-called Redfield-Hall scalar product) on the ring of symmetric functions, see e.g. [53], Chapter I, Section 4.

In particular, if $\lambda^{(1)}=\lambda^{(2)}=\emptyset$, then $K_{A^{(1)}, A^{(2)}, \theta}^{\nu}(0)$ is equal to the $L R$ number $c_{\Lambda^{(1)}, \Lambda^{(2)}}^{\nu}$.

(\$p) If $\lambda^{(1)}=\cdots=\lambda^{(k)}=\emptyset$, then the number $K_{A^{(1)}, \ldots, A^{(k)}, \theta}^{\nu}(1)$ is equal to the number of semistandard $k$-rim hook tableaux of content $\nu$ and a certain shape, see details in Section 5.2.

\section{Conjecture 6.22 (Strong $q$-log concavity conjecture for polyno-} mials $\left.K_{A^{(1)}, \ldots, A^{(k)}, \theta}^{\nu}(q)\right)$.

$\left\{K_{m A^{(1)}, \ldots, m A^{(k)}, \theta}^{m \nu}(q)\right\}_{m \geq 1}$ is a strong $q$-log concave sequence.

In particular,

$$
\begin{aligned}
& \left(K_{m A^{(1)}, \ldots, m A^{(k), \theta}}^{m \nu}(q)\right)^{2} \\
& \geq K_{(m+1) A^{(1)}, \ldots,(m+1) A^{(k)}, \theta}^{(m+1) \nu}(q) K_{(m-1) A^{(1)}, \ldots,(m-1) A^{(k)}, \theta}^{(m-1) \nu}(q) .
\end{aligned}
$$


Now we are going to state a generalization of the Fomin-Fulton-Li-Poon conjectures I and II, concerning the $L R$-numbers $c_{A, B}^{\nu}$, see $[18,60]$. To start with, we need a bit more notation from the papers quoted above.

(\$) For an ordered $k$-tuples $\left(\lambda^{(1)}, \ldots, \lambda^{(k)}\right)$ of partitions with the same number of components $p$, let $\gamma=\cup_{j=1}^{k} \lambda^{(j)}=\left(\gamma_{1} \geq \gamma_{2} \geq \cdots \geq \gamma_{k p}\right)$ be the decreasing rearrangement of the $\lambda_{i}^{(j)}{ }^{\prime} s, 1 \leq j \leq k, 1 \leq i \leq p$. Define partitions

$$
\widetilde{\lambda}^{(j)}=\left(\gamma_{j}, \gamma_{j+k}, \gamma_{j+2 k}, \ldots, \gamma_{j+(p-1) k}\right), 1 \leq j \leq k .
$$

Now suppose that $\left(A^{(1)}=\Lambda^{(1)} \backslash \lambda^{(1)}, \ldots, A^{(k)}=\Lambda^{(k)} \backslash \lambda^{(k)}\right)$ is an ordered $k$-tuples of skew diagrams and $\theta$ is a composition. Construct a new ordered $k$-tuples $\left(\widetilde{\Lambda}^{(1)}, \ldots, \widetilde{\Lambda}^{(k)}\right)$ from the $k$-tuples $\left(\Lambda^{(1)}, \ldots, \Lambda^{(k)}\right)$, and $\left(\widetilde{\lambda}^{(1)}, \ldots, \widetilde{\lambda}^{(k)}\right)$ from the $k$-tuples $\left(\lambda^{(1)}, \ldots, \lambda^{(k)}\right)$. It is easy to see that $\widetilde{\lambda}^{(j)} \subset \widetilde{\Lambda}^{(j)}, \forall j$. Finally, define $\widetilde{A}^{(j)}=\widetilde{\Lambda}^{(j)} \backslash \widetilde{\lambda}^{(j)}, 1 \leq j \leq k$ and $\widetilde{\eta}=\left(\widetilde{\lambda}_{1}^{(k)}+\sum_{j \geq 2}^{k} \widetilde{\Lambda}_{1}^{(j)}, \theta\right)$.

It is useful to consider the following modification of the above construction. Namely, for any an ordered $k$-tuples $\left(\lambda^{(1)}, \ldots, \lambda^{(k)}\right)$ of partitions with the same number of components, define a new ordered $k$-tuples of partitions $\left(\lambda^{\dagger}(1), \ldots, \lambda^{\dagger(k)}\right):=\left(\left(\widetilde{\lambda^{(1)^{\prime}}}\right)^{\prime}, \ldots, \widetilde{\left(\lambda^{(k)^{\prime}}\right)^{\prime}}\right)$. In a similar way, for an ordered $k$-tuples $\left(A^{(1)}, \ldots, A^{(k)}\right)$ of skew diagrams one can define a new an ordered $k$-tuples of skew diagrams $\left(A^{\dagger^{(1)}}, \ldots, A^{\dagger^{(k)}}\right)$.

Remember that for any partition $\lambda$ the symbol $\lambda^{\prime}$ stands for the conjugate of the partition $\lambda$.

(\$) For an ordered pair $(\lambda, \mu)$ of partitions with the same number of components, define a new ordered pair $\left(\lambda^{*}, \mu^{*}\right)$ as follows:

$\lambda_{k}^{*}=\lambda_{k}-k+\#\left\{j \mid \mu_{j}-j \geq \lambda_{k}-k\right\}, \quad \mu_{j}^{*}=\mu_{j}-j+1+\#\left\{k \mid \lambda_{k}-k>\mu_{j}-j\right\}$.

One can show, see [18], that $\lambda^{*}$ and $\mu^{*}$ are partitions and $\left|\lambda^{*}\right|+\left|\mu^{*}\right|=|\lambda|+|\mu|$.

Now suppose that $A=\Lambda \backslash \lambda, B=M \backslash \mu$ are two skew shapes and $\theta$ is a composition. Construct ${ }^{2} \Lambda^{*}$ and $M^{*}$ from the pair $(\Lambda, M)$, and $\lambda^{*}$ and $\mu^{*}$ from the pair $(\lambda, \mu)$. It is not difficult to see that $\lambda^{*} \subset \Lambda^{*}$ and $\mu^{*} \subset M^{*}$. Finally, define $A^{*}=\Lambda^{*} \backslash \lambda^{*}, B^{*}=M^{*} \backslash \mu^{*}$ and $\eta^{*}=\left(\lambda_{1}^{*}+M_{1}^{*}, \theta\right)$.

Similarly to the previous case $(\boldsymbol{A})$, for an ordered pair $(\lambda, \mu)$ of partitions, construct a new ordered pair of partitions $\left(\lambda^{\ddagger}, \mu^{\ddagger}\right):=\left(\left(\left(\lambda^{\prime}\right)^{*}\right)^{\prime},\left(\left(\mu^{\prime}\right)^{*}\right)^{\prime}\right)$, and for an ordered pair $(A, B)$ of skew diagrams define a new pair of skew diagrams $\left(A^{\ddagger}, B^{\ddagger}\right)$.

\footnotetext{
${ }^{2}$ As we learned from the referee, a similar construction was also considered by F. Bergeron, R. Biagnoli and M. Rosas, see e.g. [6, 7], or [55].
} 
(a) One can show, cf [18], Section 5.1, that

$$
\text { if }\left(A^{*}, B^{*}\right)=(A, B) \text {, then }\left(A^{*}, B^{*}\right)=\sigma(\widetilde{A}, \widetilde{B}) ; \quad\left((\tilde{A})^{*},(\tilde{B})^{*}\right)=\sigma(\widetilde{A}, \widetilde{B}) \text {, }
$$

where $\sigma$ denotes the twist $\sigma(X, Y)=(Y, X) .{ }^{3}$

Let us remark that the transformation $(\lambda, \mu) \rightarrow\left(\lambda^{*}, \mu^{*}\right):=(\lambda, \mu)^{*}$ is not one-to-one in general, e.g. $((4,4),(5,3,1))^{*}=((5,4),(4,2,1))^{*}=((4,3)$, $(5,4,1))^{*}$.

(M) For an ordered $k$-tuples $\left(\lambda^{(1)}, \ldots, \lambda^{(k)}\right)$ of partitions with the same number of components $p$, define a new ordered $k$-tuples of partitions $\left(\left\lceil\lambda^{(1)}\right\rceil, \cdots,\left\lceil\lambda^{(k)}\right\rceil\right), \operatorname{cf}(6.65)$, as follows:

$$
\left(\left\lceil\lambda^{(j)}\right\rceil\right)_{i}=\left[\left(\sum_{s=1}^{k} \lambda_{i}^{(s)}+k-j\right) / k\right], 1 \leq j \leq k, 1 \leq i \leq p .
$$

Now suppose that $\left(A^{(1)}=\Lambda^{(1)} \backslash \lambda^{(1)}, \ldots, A^{(k)}=\Lambda^{(k)} \backslash \lambda^{(k)}\right)$ is an ordered $k$-tuples of skew diagrams and $\theta$ is a composition. Construct in an obvious way a new ordered $k$-tuples of skew diagrams $\left(\left\lceil A^{(1)}\right\rceil, \cdots,\left\lceil A^{(k)}\right\rceil\right)$ from the $k$-tuples $\left(\Lambda^{(1)}, \ldots, \Lambda^{(k)}\right)$ and that $\left(\lambda^{(1)}, \ldots, \lambda^{(k)}\right)$, and put $\eta=\left(\lambda_{1}^{(1)}+\sum_{j \geq 2}^{k} \Lambda_{1}^{(j)}, \theta\right)$.

By analogy with the case $(\mathbf{\uparrow})$, for any an ordered $k$-tuples $\left(\lambda^{(1)}, \ldots, \lambda^{(k)}\right)$ of partitions with the same number of components, define a new ordered $k$ tuples of partitions

$$
\left(\lambda^{\#(1)}, \ldots, \lambda^{\#(k)}\right):=\left(\left(\left\lceil\lambda^{(1) \prime}\right\rceil\right)^{\prime}, \ldots,\left(\left\lceil\lambda^{(k) \prime}\right\rceil\right)^{\prime}\right) .
$$

In a similar way, for an ordered $k$-tuples $\left(A^{(1)}, \ldots, A^{(k)}\right)$ of skew diagrams one can define a new ordered $k$-tuples of skew diagrams $\left(A^{\#(1)}, \ldots, A^{\#}{ }^{(k)}\right)$.

Theorem 6.23. For an ordered $k$-tuples of skew diagrams $\left(A^{(1)}, \ldots\right.$, $\left.A^{(k)}\right)$ we have the following equalities:

$$
\begin{aligned}
\left(\left\lceil A^{(1)}\right\rceil, \ldots,\left\lceil A^{(k)}\right\rceil\right) & =\left(A^{\dagger}(1), \ldots, A^{\dagger}(k)\right),\left(\widetilde{A}^{(1)}, \ldots, \widetilde{A}^{(k)}\right) \\
& =\left(A^{\#(1)}, \ldots, A^{\#}(k)\right) .
\end{aligned}
$$

Conjecture 6.24 $(\checkmark)$ (The generalized Fomin-Fulton-Li-Poon conjecture I, cf $[18,60]$, and (6.66)).

Let $A^{(1)}, \ldots, A^{(k)}$ be skew diagrams, $\theta$ be a composition and $\nu$ be a partition. Then

$$
K_{\widetilde{A}^{(1)}, \ldots, \widetilde{A}^{(k)}, \theta}^{\nu}(q) \geq K_{A^{(1)}, \ldots, A^{(k)}, \theta}^{\nu}(q) .
$$

\footnotetext{
${ }^{3}$ As it was pointed by the referee, the equalities (6.69) was also proved by F. Bergeron, R. Biagnoli and M.Rosas, see e.g. [6, 7].
} 
Equivalently,

$$
K_{\left\lceil A^{(1)}\right\rceil, \ldots,\left\lceil A^{(k)}\right\rceil, \theta}^{\nu}(q) \geq K_{A^{(1)}, \ldots, A^{(k)}, \theta}^{\nu}(q) .
$$

In particular, $c_{\widetilde{A}^{(1)}, \ldots, \widetilde{A}^{(k)}}^{\nu} \geq c_{A^{(1)}, \ldots, A^{(k)}}^{\nu}, \quad c_{A^{\dagger(1)}, \ldots, A^{\dagger(k)}}^{\nu} \geq c_{A^{(1)}, \ldots, A^{(k)}}^{\nu}$.

(d) We see that the generalized Fomin-Fulton-Li-Poon conjecture I, (6.71), is equivalent to our conjecture (6.67), which in turn, is a generalization of that (6.66). As it was mentioned, in the case when $(\lambda+\mu) / 2$ is a partition, the conjecture (6.66) was stated by A. Okounkov, [60]. $[18])^{4}$

$(\downarrow)$ (The generalized Fomin-Fulton-Li-Poon conjecture II, cf

Let $A, B, \Lambda, M, \lambda, \mu$ and $\theta$ be as in (M), then

$$
K_{A^{*}, B^{*}, \theta}^{\nu}(q) \geq K_{A, B, \theta}^{\nu}(q)
$$

In particular, $\quad c_{A^{*}, B^{*}}^{\nu} \geq c_{A, B}^{\nu}, \quad c_{A^{\ddagger}, B^{\ddagger}}^{\nu} \geq c_{A, B}^{\nu}$.

\section{$(\downarrow \downarrow)$ (The generalized Okounkov conjecture II)}

Let $\left(A^{(1)}, \ldots, A^{(k)}\right)$ be an ordered $k$-tuples of skew diagrams, $\nu$ be a partition and $\theta$ be a composition. Let $p_{1}, \ldots, p_{k}$ be non-negative rational numbers, $p_{1}+\cdots+p_{k}=1$. Define $\check{A}^{(i)}=\sum_{j=1}^{k} p_{k+1-i+j} A^{(j)}, 1 \leq i \leq k$. Assume that the all $\check{A}^{(1)}, \ldots, \check{A}^{(k)}$ are skew diagrams. Then

$$
K_{\check{A}^{(1)}, \ldots, \check{A}^{(k)}, \theta}^{\nu}(q) \geq K_{A^{(1)}, \ldots, A^{(k)}, \theta}^{\nu}(q) .
$$

In particular, $c_{\check{A}^{(1)}, \ldots, \check{A}^{(k)}}^{\nu} \geq c_{A^{(1)}, \ldots, A^{(k)}}^{\nu}$.

Examples 6.25. We elucidate Conjecture 6.23 in the case $k=2$. To simplify notation we will write $A, B, \Lambda, M, \lambda$ and $\mu$ instead of $A^{(1)}, A^{(2)}, \Lambda^{(1)}$, $\Lambda^{(2)}, \lambda^{(1)}$ and $\lambda^{(2)}$ correspondingly.

(i) Take $\Lambda=(5,1), M=(4,3,1), \nu=(6,5,2,1), \theta=\left(1^{4}\right)$ and $\lambda=\mu=\emptyset$. It is easy to check that

$$
\begin{aligned}
& (\widetilde{\Lambda}, \widetilde{M})=((5,3,1),(4,1))=\left(\Lambda^{\#}, M^{\#}\right) ;\left(\Lambda^{*}, M^{*}\right)=((4,1),(5,3,1)) ; \\
& (\lceil\Lambda+M\rceil,[\Lambda+M])=((5,2,1),(4,2))=\left(\Lambda^{\dagger}, M^{\dagger}\right) ; \\
& \left(\Lambda^{\ddagger}, M^{\ddagger}\right)=((4,2),(5,2,1)) \text { and } \eta=\left(4,1^{4}\right) .
\end{aligned}
$$

Using the fermionic formula (5.44) for Kostka-Foulkes polynomials, one can find that

\footnotetext{
${ }^{4}$ As it was pointed by the referee, a generalization of the original Fomin-Fulton-Li-Poon conjecture II, [18], Conjecture 5.1, to the case of skew diagrams has been stated also by F. Bergeron, R. Biagnoli and M. Rosas, see e.g. [6, 7]; see also [55].
} 
$K_{\Lambda^{*}, M^{*}, \theta}^{\nu}(q)=K_{\widetilde{\Lambda}, \widetilde{M}, \theta}^{\nu}(q)=(3,11,18,17,11,4,1), K_{\lceil\Lambda+M\rceil,[\Lambda+M], \theta}^{\nu}(q)=$ $(3,12,19,18,11,4,1)$, $K_{\Lambda, M, \theta}^{\nu}(q)=(1,6,12,14,10,4,1)$.

Therefore, the difference $K_{\widetilde{\Lambda}, \widetilde{M}, \theta}^{\nu}(q)-K_{\Lambda, M, \theta}^{\nu}(q)$ is equal to $(2,5,6,3,1)$.

Similar computations show that if we take $\theta_{1}=\left(1^{2}, 2\right)$, then (with $\eta_{1}=$ $\left.\widetilde{\eta}_{1}=\left(4,1^{2}, 2\right)\right)$ and

$K_{\Lambda^{*}, M^{*}, \theta_{1}}^{\nu}(q)=K_{\widetilde{\Lambda}, \widetilde{M}, \theta_{1}}^{\nu}(q)=(3,9,13,10,5,1), K_{\Lambda, M, \theta_{1}}^{\nu}(q)=(1,5,9,9,5,1)$

$K_{\lceil\Lambda+M\rceil,[\Lambda+M], \theta_{1}}^{\nu}(q)=(3,10,14,11,5,1)$.

(ii) Take $A=(5,5,2,2) \backslash(3,1), B=(1,1) \backslash(1), \nu=(5,3,2,1)$ and $\theta=\left(1^{4}\right)$. It is easy to check that

$$
\begin{aligned}
& (\widetilde{A}, \widetilde{B})=((5,2,1) \backslash(3,1),(5,2,1) \backslash(1))=\left(A^{\#}, B^{\#}\right) ; \\
& \left(A^{*}, B^{*}\right)=((4,3,1) \backslash(2),(3,2,2,1) \backslash(2,1)) ;\left(A^{\ddagger}, B^{\ddagger}\right)=((2,2,1) \backslash(1),(5,4, \\
& 1,1) \backslash(3,1)) ; \\
& (\lceil A+B\rceil,[A+B])=((3,3,1,1) \backslash(2,1),(3,3,1,1) \backslash(2))=\left(A^{\dagger}, B^{\dagger}\right) .
\end{aligned}
$$

Using the fermionic formula (5.44) for Kostka-Foulkes polynomials, one can find that

$K_{A^{*}, B^{*}, \theta}^{\nu}(q)=(33,82,86,53,21,6,1), K_{\lceil A+B\rceil,[A+B], \theta}^{\nu}(q)=(12,20,14$,

$5,1)$,

$K_{\widetilde{A}, \widetilde{B}, \theta}^{\nu}(q)=(20,86,139,131,86,43,17,5,1), K_{A^{\ddagger}, B^{\ddagger}, \theta}^{\nu}(q)=(22,56,61,40$, $17,5,1)$,

$K_{A, B, \theta}^{\nu}(q)=(4,9,9,4,1)$.

Similar computations show that if we take $\eta_{1}=(1,2,1)$, then

$\left.K_{A^{*}, B^{*}, \theta_{1}}^{\nu}(q)=(33,64,41,9), K_{A, B, \theta_{1}}^{\nu}(q)=(4,7,3)\right), K_{\lceil A+B\rceil,[A+B], \theta_{1}}^{\nu}(q)$ $=(12,15,5)$,

$K_{\widetilde{A}, \widetilde{B}, \theta_{1}}^{\nu}(q)=(20,73,87,49,13,1), K_{A^{\ddagger}, B^{\ddagger}, \theta_{1}}^{\nu}(q)=(22,45,32,9)$.

These examples show that, probably, there are no simple relationships between polynomials $K_{A^{*}, B^{*}, \theta}^{\nu}(q), K_{\widetilde{A}, \widetilde{B}, \theta}^{\nu}(q), K_{A^{\ddagger}, B^{\ddagger}, \theta}^{\nu}(q)$ and $K_{\lceil A+B\rceil,[A+B], \theta}^{\nu}(q)$.

( However, based on examples, we expect that $\max \left\{c_{A^{*}, B^{*}}^{\nu}, c_{\widetilde{\Lambda}, \widetilde{M}}^{\nu}\right\} \geq$ $c_{\lceil A+B],[A+B]}^{\nu}$.

(4) We expect that if $\theta_{1}$ and $\theta_{2}$ are compositions such that $\theta_{2}$ is a subdivision of $\theta_{1}$, see Section 1 , Notation, then

$$
\begin{aligned}
& K_{\widetilde{A}^{(1)}, \ldots, \widetilde{A}^{(k)}, \theta_{2}}^{\nu}(q)-K_{A^{(1)}, \ldots, A^{(k)}, \theta_{2}}^{\nu}(q) \\
& \quad \geq K_{\widetilde{A}^{(1)}, \ldots, \widetilde{A}^{(k)}, \theta_{1}}^{\nu}(q)-K_{A^{(1)}, \ldots, A^{(k)}, \theta_{1}}^{\nu}(q) \geq 0,
\end{aligned}
$$




$$
\begin{aligned}
& K_{A^{*}, M^{*}, \theta_{2}}^{\nu}(q)-K_{A, B, \theta_{2}}^{\nu}(q) \\
& \quad \geq K_{A^{*}, B^{*}, \theta_{1}}^{\nu}(q)-K_{A, B, \theta_{1}}^{\nu}(q) \geq 0, \\
& K_{A^{\ddagger}, M^{\ddagger}, \theta_{2}}^{\nu}(q)-K_{A, B, \theta_{2}}^{\nu}(q) \\
& \quad \geq K_{A^{\ddagger}, B^{\ddagger}, \theta_{1}}^{\nu}(q)-K_{A, B, \theta_{1}}^{\nu}(q) \geq 0, \\
& K_{\left\lceil A^{(1)}\right\rceil, \ldots,\left[A^{k)}\right], \theta_{2}}^{\nu}(q)-K_{A^{(1)}, \ldots, A^{(k)}, \theta_{2}}^{\nu}(q) \\
& \quad \geq K_{\left\lceil A^{(1)}\right\rceil, \ldots,\left[A^{(k)}\right], \theta_{1}}^{\nu}(q)-K_{A^{(k)}, \ldots, A^{(k)}, \theta_{1}}^{\nu}(q) \geq 0, \\
& K_{\check{A}^{(1)}, \ldots, \check{A}^{(k)}, \theta_{2}}^{\nu}(q)-K_{A^{(1)}, \ldots, A^{(k)}, \theta_{2}}^{\nu}(q) \\
& \quad \geq K_{\check{A}^{(1)}, \ldots, \check{A}^{(k)}, \theta_{1}}^{\nu}(q)-K_{A^{(1)}, \ldots, A^{(k)}, \theta_{1}}^{\nu}(q) \geq 0 .
\end{aligned}
$$

Remark 6.26. We expect that Conjecture 6.3 (Positivity), Conjecture 6.5 (Saturation), Conjecture 6.7 (Rationality), Conjecture 6.10 (Polynomiality), Conjecture 6.17 ( $q$-Log concavity and $P$-positivity), Conjecture 6.24 (Generalized Fomin-Fulton-Li-Poon's conjectures I and II) are still valid for the level $l$-restricted parabolic Kostka polynomials $K_{\lambda \mu \eta}^{(l)}(q)$, see Remark 4.28 for the definition of the latter.

\section{§6.9. Miscellany}

Conjecture 6.27 (Rationality conjecture for the $L L T q$-analog of $L R$-numbers).

Let $\lambda, \mu$ and $\nu$ be partitions, and $c_{\lambda, \mu}^{\nu}(q)$ stands for the $q$-analog of Littlewood-Richardson numbers defined in [11], [47]. Then

$$
\sum_{n \geq 0} c_{n \lambda, n \mu}^{n \nu}(q) t^{n}=P_{\lambda, \mu}^{\nu}(q, t) / Q_{\lambda, \mu}^{\nu}(q, t),
$$

where $P_{\lambda, \mu}^{\nu}(q, t)$ and $Q_{\lambda, \mu}^{\nu}(q, t)$ are mutually prime polynomials with integer coefficients. Moreover,

$$
Q_{\lambda, \mu}^{\nu}(q, t)=\prod_{i \in I}\left(1-q^{i} t\right)^{n_{i}}
$$

for some finite set of integers $I=I_{\lambda, \mu}^{\nu}$, and a set of positive integers $n_{i}, i \in I$.

( We expect the similar conjecture for the parabolic Kazhdan-Lusztig polynomials, see e.g. [49] for the definition of the latter.

Conjecture 6.28 (Saturation conjecture for the structural constants of the multiplication of the Schubert polynomials).

For each $n \geq 1$, let $\Sigma^{(n)}$ denote the set of all permutations $w$ such that the code of $w$ has length at most $n$. Denote by $\Sigma^{(\infty)}$ the union $\bigcup_{n \geq 1} \Sigma^{(n)}$. 
If $w \in \Sigma^{(n)}$ and $N \geq 1$ is an integer, define the permutation $N * w \in \Sigma^{(N n)}$ to be a unique permutation with the code $\left(N c_{1}, \ldots, N c_{n}\right)$, where $\left(c_{1}, \ldots, c_{n}\right)$ is the code of $w$.

For each $w \in \Sigma^{(n)}$ denote by $\mathfrak{S}_{w} \in P_{n}:=\mathbb{Z}\left[x_{1}, \ldots, x_{n}\right]$ the corresponding Schubert polynomial. It is well-known that the $\mathfrak{S}_{w}, w \in \Sigma^{(n)}$, form a $\mathbb{Z}$-basis of $P_{n}$.

Finally, if $u, v$ are permutations which belong to the infinite symmetric group $\Sigma^{(\infty)}$, denote by $c_{u, v}^{w}$ the structural constants for the multiplication of Schubert polynomials:

$$
\mathfrak{S}_{u} \mathfrak{S}_{v}=\sum_{w \in \Sigma^{(\infty)}} c_{u, v}^{w} \mathfrak{S}_{w}
$$

Then

(a) $c_{N * u, N * v}^{N * w} \neq 0$ for some integer $N \geq 1$ if and only if $c_{u, v}^{w} \neq 0$.

(4) We expect that the formal power series

$$
\sum_{N \geq 1} c_{N * u, N * v}^{N * w} t^{N}
$$

is a rational function in $t$ (with the only possible pole at $t=1$ ??). In other words, the function $N \longrightarrow c_{N * u, N * v}^{N * w}$ is a polynomial in $N$ with rational (nonnegative ??) coefficients.

Problem 6.29 (Generalized saturation problem for KazhdanLusztig's polynomials).

Let $u, w \in \Sigma_{n}$ be two permutations, denote by

$$
P_{u, w}(q)=1+\cdots+d(u, w) q^{c(u, w)}, \quad d(u, w) \neq 0,
$$

the corresponding Kazhdan-Lusztig polynomial [27].

(a) Prove (or disprove) that

(1) $c(N * u, N * w)=N c(u, w)$ for any positive integer $N$;

(2) $d(N * u, N * w)=1$ for some positive integer $N$ if and only if $d(u, w)$ $=1$.

The similar Problem can be stated for the Kazhdan-Lusztig polynomials corresponding to the affine symmetric group.

However, we didn't extensively test Conjecture 6.27 and Problem 6.28 on a computer. 
We want to end this Section by the following question and problem:

Question 6.30 (A $q$-analog of the structural constants $c_{u, v}^{w}$ ).

Does there exist a natural $q$-analog $c_{u, v}^{w}(q) \in \mathbb{N}[q]$ of the structural constants $c_{u, v}^{w}$, so that $c_{u, v}^{w}=c_{u, v}^{w}(1)$, which for the grassmannian permutations $u$, $v$ and $w$ coincides with the $q$-analog $c_{\lambda(u), \lambda(v)}^{\lambda(w)}(q)$ of the LR-numbers?

Here $\lambda(w)$ denotes the shape of a permutation $w$, see [52] for a detailed account to the theory of Schubert polynomials. As for a definition of the $q$ analog $c_{\lambda, \mu}^{\nu}(q)$ of the LR-numbers, see e.g. $[11,47]$.

Problem 6.31 (Define the polynomials $c_{\lambda, \mu}^{\nu}(q)$ through the geometry of Schubert varieties).

Let $n \geq m$ be fixed positive integers, and $\lambda, \mu$ and $\nu$ be three partitions such that $\max (l(\lambda), l(\mu), l(\nu)) \leq m, \max \left(\lambda_{1}, \mu_{1}, \nu_{1}\right) \leq n$, and $|\lambda|+|\mu|=|\nu|$.

It is well-known that the LR-number $c_{\lambda, \mu}^{\nu}$ counts the number of (isolated) points in the triple intersection $\mathfrak{S}_{\lambda} \cap \mathfrak{S}_{\mu} \cap \mathfrak{S}_{\nu^{*}}$ of the Schubert varieties $\mathfrak{S}_{\lambda}$, $\mathfrak{S}_{\mu}$ and $\mathfrak{S}_{\nu^{*}}$ in the Grassmannian variety $G(m, n+m)$, see e.g. [19] for the explanations of omitted notation, definitions and details.

(\$) Find a geometric way to attach to each intersection point $x \in \mathfrak{S}_{\lambda} \cap$ $\mathfrak{S}_{\mu} \cap \mathfrak{S}_{\nu^{*}}$ an integer number $c(x)$ such that the generating function

$$
\sum_{x \in \mathfrak{S}_{\lambda} \cap \mathfrak{S}_{\mu} \cap \mathfrak{S}_{\nu^{*}}} q^{c(x)}
$$

coincides with the LLT q-analog $c_{\lambda, \mu}^{\nu}(q)$ of the Littlewood-Richardson number $c_{\lambda, \mu}^{\nu}$

\section{Acknowledgements}

This note presents an extended version of my talks given at

- The International Institute for Advanced Study, Japan, January 29-30 1999; The International Workshop "Physical Combinatorics".

- The Nagoya University, Japan, August 23-27; The International Workshop "Physics and Combinatorics".

- The Erwin Schroedinger International Institute of Mathematical Physics, Vienna, Austria, July 2-15, 2000; The International Workshop "Representation Theory 2000".

- The Isaac Newton Institute for Mathematical Sciences, Cambridge, UK, June 28-July 4, 2001; The International Workshop "Symmetric Functions and Macdonald Polynomials". 
- The Research Institute for Mathematical Sciences, Kyoto, Japan, August 12-16, 2001; The International Workshop "Integrable Models, Combinatorics and Representation Theory".

- The Research Institute for Mathematical Sciences, Kyoto, Japan, May 22, 2002; Colloquium talk.

- The Kyushu University, Japan, November 17-21, 2003; The Second East Asian Conference on Algebra and Combinatorics.

I am grateful to the organizes of these Workshops and Conference for a kind invitation, financial support and the opportunity to talk about one of my the most favorite subjects.

Finally, I would like to thank the referee for many valuable remarks and comments, in particular, for sending the references on the preprints $[6,7]$ and [55], which have some overlaps with the content of our Section 6.8.

\section{References}

[1] Agaoka, Y., An algorithm to calculate plethysms of Schur functions and the table up to total degree 16, Tech. Rep. 46, October 1995, Hiroshima University.

[2] Baldoni-Silva, W. and Vergne, M., Residues formulae for volumes and Ehrhart polynomials of convex polytopes, Preprint, 2001, CO/0103097.

[3] Beck Matthias and Pixton, D., The Ehrhart polynomial of the Birkhoff polytope, Preprint, 2002, CO/0202267.

[4] Belkale P., Geometric proofs of Horn and saturation conjectures, Preprint, 2002, AG/0208107.

[5] Berezin, F. and Gelfand, I. M., Some remarks on the theory of spherical functions on symmetric Riemannian manifolds, (Russian) Trudy Moskov. Mat. Ob., 5 (1956), 311-351.

[6] Bergeron, F., On a conjecture concerning Littlewood-Richardson coefficients, Conference transparencies (http://bergeron.math.uqam.ca/dartmouth.pdf).

[7] Bergeron, F., Biagnoli, R. and Rosas, M., Inequalities between Littlewood-Richardson coefficients, Preprint, 2004, CO/0403541.

[8] Billey, S., Guillemin, V. and Rassart, E., A vector partition function for the multiplicities $s l_{k}(\mathbb{C})$, Preprint, 2003, CO/0307227.

[9] Buch Anders Skovsted, The saturation conjecture (after A. Knutson and T. Tao). With an appendix by William Fulton, Enseign. Math. (2), 46 (2000), 43-60.

[10] Butler, L., Subgroup Lattices and Symmetric Functions, Mem. AMS, 539 (1994).

[11] Carre, C. and Leclerc, B., Splitting the square of a Schur function into its symmetric and antisymmetric parts, J. Algebraic Comb., 4 (1995), 201-231.

[12] De Loera, J. and McAllister, T., Vertices of Gelfand-Tsetlin polytopes, Preprint $\mathrm{CO} / 0309329$.

[13] De Loera, J. and Sturmfels, B., Algebraic unimodal counting, Algebraic and geometric methods in discrete optimization. Math. Program. Ser. B, 96 (2003), 183-203.

[14] Derksen, H. and Weyman, J., Semi-invariants of quivers and saturation for LittlewoodRichardson coefficients, J. Amer. Math. Soc., 13 (2000), 467-479.

[15] - On the Littlewood-Richardson polynomials, J. Algebra 255, (2002), 247-257.

[16] Désarménien, J., Leclerc, B. and Thibon, J.-Y., Hall-Littlewood functions and KostkaFoulkes polynomials in representation theory, Sem. Lothar. Combin., 32, (1994)(electronic). 
[17] Dvir Yoav, On the Kronecker product of $S_{n}$ characters, J. Algebra, 154 (1993), 125-140.

[18] Fomin, S., Fulton, W., Li Chi-Kwong and Poon Yiu-Tung, Eigenvalues, singular values, and Littlewood-Richardson coefficients, Preprint, 2003, AG/0301307.

[19] Fulton, W., Young Tableaux, Cambridge Univ. Press, Cambridge, 1997.

[20] Eigenvalues, invariant factors, highest weights, and Schubert calculus, Bull. Amer. Math. Soc. (N.S.), 37 (2000), 209-249.

[21] Eigenvalues of sums of Hermitian matrices (after A. Klyachko), Séminaire Bourbaki. Vol. 1997/98. Astérisque, 252 (1998), Exp. No. 845, 5, 255-269.

[22] Hatayama, G., Kirillov, A. N., Kuniba, A., Okado, M., Takagi, T. and Yamada, Y. Character formulae of $\widehat{s} l(n)$-modules and inhomogeneous paths, Nucl. Phys. B, 536 (1999), 575-616.

[23] Heckman, G. J., Projections of orbits and asymptotic behavior of multiplicities for compact connected Lie groups, Invent. Math., 67 (1982), 333-356.

[24] Hibi, T., Algebraic combinatorics on convex polytope, Carslaw Publications, 1992.

[25] Hotta, R. and Shimomura, N., The fixed-point subvarieties of unipotent transformations on generalized flag varieties and the Green functions. Combinatorial and cohomological treatments, Math. Ann., 241 (1979), 193-208.

[26] Kac, V., Infinite dimensional Lie algebras, 3rd ed., Cambridge Univ. Press, 1994.

[27] Kazhdan, D. and Lusztig, G., Representations of Coxeter groups and Hecke algebras, Invent. Math., 53 (1979), 165-184.

[28] Kirillov, A. N., Completeness of the states for the generalized Heisenberg model (Russian), Zap. Nauch. Semin. LOMI, 134 (1985), 169-189, translation in J. Soviet Math., 36 (1987), 115-128.

[29] Geom. Phys., 5 (1988), 365-389.

[30] Unimodality of generalized Gaussian coefficients, C. R. Acad. Sci. Paris, Serie I, 315 (1992), 497-501.

[31] Dilogarithm identities, Progr. Theor. Phys. Suppl., 118 (1995), 61-142.

[32] Wew combinatorial formula for modified Hall-Littlewood polynomials, Contemp. Mathematics, 254 (2000), 283-333.

[33] Combinatorics of Young tableaux and configurations (Russian), Transactions of SPb Math. Soc., 7 (2000), 23-115.

[34] Kostant partition functions for the root system of type A, Preprint, 1999, 20p.

[35] - Ubiquity of Kostka polynomials, Physics and combinatorics 1999 (Nagoya), 85-200, World Sci. Publishing, River Edge, NJ, 2001.

[36] - Introduction to tropical combinatorics, Physics and combinatorics, 2000 (Nagoya), 82-150, World Sci. Publishing, River Edge, NJ, 2001.

[37] - On some quadratic algebras and Kostant partition function, in preparation.

[38] Kirillov, A. N. and Berenstein, A. D., Groups generated by involutions, Gelfand-Tsetlin patterns, and combinatorics of Young tableaux, St. Petersburg Math. J., 7 (1996), $77-$ 127 .

[39] Kirillov, A. N., Kuniba, A. and Nakanishi, T., Skew Young diagram method in spectral decomposition of integrable lattice models II: Higher levels, Nucl. Phys. B, 529 (1998), $247-271$.

[40] Kirillov, A. N. and Reshetikhin, N. Yu., The Bethe ansatz and combinatorics of the Young tableaux (Russian), Zap. Nauch. Semin. LOMI, 155 (1986), 65-115, translation in Journal of Soviet Math., 41 (1988), 925-955.

[41] Kirillov, A. N. and Shimozono, M., A generalization of the Kostka-Foulkes polynomials, J. Algebraic Combin., 15 (2002), 27-69.

[42] Kirillov, A. N., Schilling, A. and Shimozono, M., A bijection between LittlewoodRichardson tableaux and rigged configurations, Selecta Math. (N.S.), 8 (2002), 67-135. 
[43] Knutson, A. and Tao, T., The honeycomb model of GL(C ) tensor products. I. Proof of the saturation conjecture, J. Amer. Math. Soc., 12 (1999), 1055-1090.

[44] Knutson, A., Tao, T. and Woodward, C., The honeycomb model of GL(C ) tensor products II: puzzles determine facets of the Littlewood-Richardson cone, J. Amer. Math. Soc., 17 (2004), 19-48.

[45] Kostant, B., Lie group representations on polynomial rings, Amer. J. Math., 85 (1963), 327-404.

[46] Kuniba, A., Misra, K. C., Okado, M., Takagi, T. and Uchiyama, J., Characters of Demazure modules and solvable lattice models, Nucl. Phys. B, 510 (1998), 555-576.

[47] Lascoux, A., Leclerc, B. and Thibon, J.-Y., Ribbon tableaux, Hall-Littlewood functions, quantum affine algebras, and unipotent varieties, J. Math. Phys., 38 (1997), 1041-1068.

[48] Lascoux, A. and Schützenberger, M.-P., Sur une conjecture de H. O. Foulkes, $C$. $R$. Acad. Sci. Paris, 286A (1978), 323-324.

[49] Leclerc, B. and Thibon, J.-Y., Littlewood-Richardson coefficients and Kazhdan-Lusztig polynomials, Combinatorial methods in representation theory (Kyoto, 1998), Adv. Stud. Pure Math., 28 (2000), 155-220.

[50] Lidskii, B. V., The Kostant function of the system of roots $A_{n}$, (Russian) Funktsional. Anal. i Prilozhen., 18 (1984), 76-77.

[51] _ Characters of finite-dimensional irreducible representations of the Lie algebra sl(n), (Russian) Funktsional. Anal. i Prilozhen., 18 (1984), 86-87.

[52] Macdonald, I. G., Notes on Schubert polynomials, Publications du LCIM 6 (1991), Université du Québec à Montréal.

[53] Macdonald, I. G., Symmetric functions and Hall polynomials, 2nd ed., Oxford, 1995.

[54] McMullen, P., Valuations and Euler-type relations on certain class of convex polytopes, Proc. London Math. Soc., 35 (1977), 113-135.

[55] MacNamara, P., Questions of Schur-positivity, Conference transparencies (http:// www.lacim.uqam.ca/mcnamara).

[56] Merris, R., Single-hook characters and Hamiltonian circuits, Linear and Multilinear Algebra, 14 (1983), 21-35.

[57] Murnaghan, F. D., The analysis of the Kronecker product of irreducible representations of the symmetric group, Amer. J. Math., 60 (1938), 761-784.

[58] Nakayashiki, A. and Yamada, Y., On spinon character formulas, in Frontiers in Quantum Field Theory, ed. Itoyama et al., World Scientific, Singapore, 1996, 367-371.

[59] Okounkov, A., Log-concavity of multiplicities with application to characters of $\mathrm{U}(\infty)$, Adv. Math., 127 (1997), no. 2, 258-282.

[60] Why would multiplicities be log-concave?, The orbit method in geometry and physics (Marseille, 2000), Progr. Math. 213, 329-347, Birkhäuser Boston, Boston, MA, 2003.

[61] Rassart, E., A polynomiality property for Littlewood-Richardson coefficients, Preprint, 2003, CO/0308101 v2.

[62] Schilling, A., Multinomials and polynomial bosonic forms for the branching functions of the $\widehat{s u}(2)_{M} \times \widehat{s u}(2)_{N} / \widehat{s u}(2)_{M+N}$ conformal coset models, Nucl. Phys. B, 467 (1996) 247-271.

[63] Schilling, A. and Warnaar, S. Ole, Inhomogeneous lattice paths, generalized Kostka polynomials and $A_{n-1}$ supernomials, Comm. Math. Phys., 202 (1999), 359-401.

[64] Shimozono, M. and Weyman, J., Graded characters of modules supported in the closure of a nilpotent conjugacy class, European J. Combin., 21 (2000), 257-288.

[65] Speyer, D., Horn's problem, Vinnikov curves ant the hive cone, Preprint, AG/0311428.

[66] Stanley, R., Log-concave and unimodal sequences in algebra, combinatorics, and geometry, Ann. New York Acad. Sci., 576 (1989), 500-535.

[67] Enumerative Combinatorics, vols. 1 and 2, Cambridge University Press, 1999.

[68] - Acyclic flow polytopes and Kostant's partition function, Conference transparencies (www-math.mit.edu/rstan/trans.html). 
[69] Stembridge, J. R., Rational tableaux and the tensor algebra of $\mathfrak{g l}(n)$, J. Combin. Theory Ser. A., 46 (1987), 79-120.

[70] Vallejo, E., Stability of Kronecker products of irreducible characters of the symmetric group, Electron. J. Combin., 6 (1999), Research Paper 39.

[71] Warnaar, S. O., The Andrews-Gordon identities and $q$-multinomial coefficients, Comm. Math. Phys., 184 (1997), 203-232.

[72] Weyman, J., The equations of conjugacy classes of nilpotent matrices, Invent. Math., 98 (1989), 229-245.

[73] Zelevinsky, A. V., A generalization of the Littlewood-Richardson rule and the RobinsonSchensted-Knuth correspondence, J. Algebra, 69 (1981), 82-94.

[74] - Littlewood-Richardson semigroups, New perspectives in algebraic combinatorics (Berkeley, CA, 1996-97). Math. Sci. Res. Inst. Publ. 38 (1999), 337-345, Cambridge Univ. Press, Cambridge. 